\title{
A classification of localizing subcategories by relative homological algebra
}

\author{
Dissertation \\ zur Erlangung des mathematisch-naturwissenschaftlichen Doktorgrades \\ „Doctor rerum naturalium“ (Dr.rer.nat.) \\ der Georg-August-Universität Göttingen \\ im Promotionsprogramm der PhD School of Mathematical Science (SMS) \\ der Georg-August University School of Science (GAUSS) \\ vorgelegt von \\ George Nadareishvili \\ aus Tiflis, Georgien
}

Göttingen 2015 


\title{
Betreungsausschuss:
}

\author{
Betreuer: Prof. Dr. Ralf Meyer \\ Mathematisches Institut, Georg-August-Universität Göttingen \\ Betreuer: Prof. Dr. Thomas Schick \\ Mathematisches Institut, Georg-August-Universität Göttingen
}

\author{
Mitglieder der Prüfungskommission:
Referent: Prof. Dr. Ralf Meyer
Mathematisches Institut, Georg-August-Universität Göttingen
Koreferent: Prof. Dr. Thomas Schick
Mathematisches Institut, Georg-August-Universität Göttingen

Prof. Dr. Dorothea Bahns

Mathematisches Institut, Georg-August-Universität Göttingen

Prof. Dr. Preda Mihăilescu

Mathematisches Institut, Georg-August-Universität Göttingen

Prof. Dr. Karl-Henning Rehren

Institut für Theoretische Physik, Georg-August-Universität Göttingen

Prof. Dr. Max Wardetzky

Institut für Numerische und Angewandte Mathematik,

Georg-August-Universität Göttingen

Tag der mündlichen Prüfung: 16.10.2015 


\begin{abstract}
In this thesis, we use the tools of relative homological algebra in triangulated categories to define a sensible notion of support for objects in the bootstrap class $\mathcal{B}(X)$ of a Kasparov category of $\mathrm{C}^{*}$-algebras over a finite topological space $X$ with totally ordered lattice of open subsets. The category $\mathcal{B}(X)$ is equivalent to a bootstrap category of filtrations of $\mathrm{C}^{*}$-algebras.

As a consequence, we provide a full classification of localizing subcategories of $\mathcal{B}(X)$ in terms of a product of lattices of noncrossing partitions of a regular $(n+1)$-gon, where $n$ is the number of points in $X$.

In addition, we consider the 2-periodic derived category of countable modules over the ring of upper triangular $n \times n$-matrices. Since the homological algebra is the same, the lattices of localizing subcategories in this category and $\mathcal{B}(X)$ are isomorphic.
\end{abstract}




\section{Acknowledgements}

I would like to express my sincere gratitude to my main advisor Ralf Meyer, who always had time to give scientific guidance, and without whom my education in Göttingen would not have been possible.

To Tamaz Kandelaki, who, despite his serious illness, provided me with an invaluable support and advice at the early stage of my doctoral studies in Georgia.

I would also like to thank my second supervisor Thomas Schick, for interesting mathematical conversations and suggestions during my annual reporting.

I am greatly indebted to Ivo Dell'Ambrogio for his very helpful suggestions on my work during his visit in Göttingen. Also, to everyone at the Mathematics Institute, for creating the great learning atmosphere that I enjoyed during the last three years.

Finally, I am also grateful to DAAD (Deutscher Akademischer Austauschdienst) for the financial support in form of a scholarship throughout my stay in Germany. 


\section{Contents}

Contents $\quad$ v

1 Introduction 1

2 Preliminaries $\quad 7$

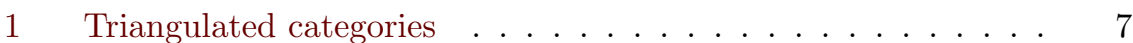

1.1 Definition of a triangulated category . . . . . . . . 7

1.2 Homotopy limits . . . . . . . . . . . . . . . . 99

1.3 Localization of a triangulated category . . . . . . . . . 10

1.4 Homological functors . . . . . . . . . . . . . . . . 11

2 Homological algebra in triangulated categories . . . . . . . 12

$2.1 \quad$ Ideals . . . . . . . . . . . . . . . . . . . . . 12

2.2 Relative projective objects and exactness . . . . . . 13

2.3 The universal I-exact functor . . . . . . . . . . . . . 13

3 Relevant examples .................... 14

3.1 The derived category of an abelian category . . . . . . 15

$3.2 \quad$ Bivariant K-theory . . . . . . . . . . . . . . . 18

$3.3 \quad \mathrm{C}^{*}$-Algebras with an action of a topological space . . . 21

3 Classification 33

4 Properties of $\mathcal{B}(X)$ and cohomological support . . . . . . 33

$4.1 \quad$ Some general results for $\mathcal{B}(X) \ldots \ldots \ldots \ldots$

4.2 Cohomological support . . . . . . . . . . . . 35

5 Localizing subcategories in the totally ordered case . . . . . . 36

$5.1 \quad$ Case of extensions . . . . . . . . . . . . . . 40

6 Classification by noncrossing partitions . . . . . . . . . . . . 42

6.1 Classification . . . . . . . . . . . . . . . 43

$7 \quad$ Algebraic analogue . . . . . . . . . . . . . 46

A Appendices $\quad \mathbf{5 5}$

A.1 Localization of a category . . . . . . . . . . . . . 55

A.1.1 General localization . . . . . . . . . . . . 55

A.1.2 Calculus of fractions . . . . . . . . . . . 56

A.2 Quiver representations . . . . . . . . . . . . 58

$\begin{array}{ll}\text { Bibliography } & 61\end{array}$ 



\section{Introduction}

A triangulated category is a category with some extra structure that allows to apply some basic tools of homotopy theory and homological algebra. Examples of such categories arise also in other areas such as representation theory and noncommutative geometry. Our main case of interest are the equivariant bivariant $\mathrm{K}$-theory categories for $\mathrm{C}^{*}$-algebras over certain finite spaces, and some derived categories that are closely related in the sense that they have the same relative homological algebra.

A main objective of pure mathematics is classification. Triangulated categories allow to classify objects up to "homological" equivalence: two objects are equivalent if they can be transformed into each other with natural operations performed in a triangulated category. In the latter case, we say that these objects generate the same thick or localizing subcategory. More precisely, a full subcategory of a triangulated category is called thick if it is closed under suspension, formation of triangles and retracts. It is localizing if, in addition, it is closed under whatever coproducts exist in the ambient category. A general classification program aims to establish a lattice isomorphism between (thick) localizing subcategories and a suitable computable lattice.

Although interesting in its own right, such a classification can also be used to obtain an interesting invariant or different structural information about a triangulated category.

Results of this nature can be found in various fields of mathematics. The first work on this classification problem was done by Michael J. Hopkins and Jeffrey H. Smith [21] in 1998. They classified thick subcategories for the $p$-local finite stable homotopy category using certain Morova K-theory functors as homological invariants. Hopkins-Smith also showed how this classification is equivalent to the earlier Nilpotence Theorem by Davinatz-Hopkins-Smith [13] and thus can be used to prove the famous Periodicity Theorem of stable homotopy theory.

As an algebraic parallel, building on the previous work of Hopkins [20], Amnon Neeman [33] classified all localizing subcategories of the unbounded derived category $\mathfrak{D} \mathfrak{e r}(R)$ of $R$-modules, where $R$ is a commutative and noetherian ring. He proved that there is an inclusion-preserving isomorphism between localizing subcategories of $\mathfrak{D e r}(R)$ and the set of all subsets of the space $\operatorname{Spec} R$. This isomorphism also involves some aspects of the topology on Spec $R$, as it restricts to a bijection between sets closed under specialization on one side, and those localizing subcategories for which localization commutes with direct sums on the other side. This result by Hopkins-Neeman is rather remarkable, as it recovers the very insightful object $\operatorname{Spec} R$ out of something seemingly so abstract and algebraic like $\mathfrak{D e r}(R)$.

In representation theory, Benson, Iyengar and Krause [6] classify the lo- 
calizing subcategories of the stable module category of the group algebra $k G$, where the group $G$ is finite and the characteristic of the field $k$ divides the order of $G$. This classification is in terms of subsets of the projective scheme $\operatorname{Proj}\left(H^{*}(G ; k)\right)$.

In favorable cases, like when we have a monoidal structure, the common theme of recovering a space out of a triangulated category was formalized by Paul Balmer [3]. The classification methods can also be generalized for certain compactly generated triangulated categories as explained below.

\section{Support theory}

The classification of localizing subcategories always proceeds by defining a notion of support for objects in a triangulated category. This is usually a canonical process of assigning a subset of a certain space to every object. Generally speaking, the support introduces a geometric approach for studying an algebraic structure.

Once we have a good definition of support, the classification result should say, first, that any subcategory is determined uniquely by the supports of its objects; secondly, it should describe the sets that appear as supports of localizing subcategories.

If one works with a compactly generated triangulated category with small coproducts and with an action of a commutative noetherian ring $R$, BensonIyengar-Krause [5] define supports based on a construction of local cohomology functors with respect to the ring $R$. Then $\operatorname{Spec} R$ naturally serves as a locus for supports. This method is rather powerful, and classifications like [33], [6] and a few others fall under this theory. However, the triangulated categories we are interested in are not compactly generated in the usual sense because they do not have arbitrary small coproducts. This obstruction is not trivial, since a very crucial fact used by Benson-Iyengar-Krause, namely the classical Brown representability, does not hold. In addition, in our case, any ring $R$ that acts on a category and is large enough to accommodate a sensible notion of support is noncommutative, and therefore there is no good candidate for $\operatorname{Spec} R$.

Below we describe our approach.

\section{Setup and results}

We will be dealing with two kinds of triangulated categories: the Kasparov category of $\mathrm{C}^{*}$-algebras with an action of a topological space and the 2-periodic derived category of the ring of upper triangular matrices. We briefly explain both setups and the main results in both cases.

\section{The bootstrap category $\mathcal{B}(X)$}

A $\mathrm{C}^{*}$-algebra over a topological space $X$, shortly an $X$-C*-algebra, is a pair $(A, \psi)$, where $A$ is a $\mathrm{C}^{*}$-algebra and $\psi: \operatorname{Prim}(A) \rightarrow X$ a continuous map. $\mathfrak{K} \mathfrak{K}(X)$ is defined to be the Kasparov category of $\mathrm{C}^{*}$-algebras over $X$ : its objects are 
separable $\mathrm{C}^{*}$-algebras over $X$, its morphism set from $A$ to $B$ is $\mathrm{KK}_{0}(X ; A, B)$ : an $X$-equivariant version of Kasparov's bivariant $\mathrm{K}$-group in degree zero [30]. The composition is given by the corresponding Kasparov product.

As demonstrated by Meyer-Nest [30], $\mathfrak{K} \mathfrak{K}(X)$ is a triangulated category.

From now on, unless stated otherwise, assume $X$ to be finite, $T_{0}$, with totally ordered lattice of open subsets. Let $n=|X|$. Then a $\mathrm{C}^{*}$-algebra over $X$ is equivalent to a $\mathrm{C}^{*}$-algebra with an increasing chain of ideals

$$
\{0\}=I_{0} \triangleleft I_{1} \triangleleft I_{2} \triangleleft \cdots \triangleleft I_{n}=A .
$$

The bootstrap category $\mathcal{B}(X)$ is defined as the smallest localizing subcategory of $\mathfrak{K} \mathfrak{K}(X)$ which contains all the possible ways $\mathbb{C}$ can be made into a $\mathrm{C}^{*}$-algebra over $X$ [30]. Another description of the bootstrap subcategory was also derived by Meyer-Nest [32]: a $\mathrm{C}^{*}$-algebra over $X$ belongs to $\mathcal{B}(X)$ if and only if it satisfies an appropriate Universal Coefficient Theorem. This will be recalled in Section 3 .

Classification for the bootstrap class. If the space $X$ is just a single point, we recover the original definitions of Kasparov's $\mathfrak{K} \mathfrak{K}$ category and a bootstrap class $\mathcal{B}$, characterized by the classical Universal Coefficient Theorem by Rosenberg and Schochet [36]. Brown representabiliy already fails here, but we still have the action of a commutative noetherian endomorphism ring $\mathbb{Z} \cong \operatorname{End}_{\mathcal{B}}(\mathbb{C})$ of the tensor unit object $\mathbb{C} \in \mathcal{B}$.

Ivo Dell'Ambrogio [12] classified localizing subcategories of the bootstrap class $\mathcal{B}$ in terms of subsets of the spectrum of this ring of integers. As in [33] and [5], to define supports for objects in $\mathcal{B}$ Dell'Ambrogio uses the collection of functors $\mathbf{C}_{\mathcal{B}}=\left\{\mathrm{K}_{*}\left(-; \mathbb{F}_{p}\right) \mid p \in \operatorname{Spec} \mathbb{Z}\right\}$, where $\mathrm{K}_{*}\left(-; \mathbb{F}_{p}\right)$ is the K-theory with coefficients in the residue field $\mathbb{F}_{p}$; that is $\mathbb{F}_{p}=\mathbb{Z} / p$ for $p \neq 0$ and $\mathbb{F}_{p}=\mathbb{Q}$ for $p=0$. More precisely, the support of the object $A \in \mathcal{B}$ is the subset of $\operatorname{Spec} \mathbb{Z}$ for which the corresponding functors in $\mathbf{C}_{\mathcal{B}}$ do not vanish on $A$.

We are going to generalize this classification result to $\mathcal{B}(X)$, where there is no action of a large enough commutative ring.

Main results for $\mathcal{B}(X)$. Let $Y \subseteq X$ be a locally closed subset; that is, a subset that is a difference of two open sets in $X$. There are exactly $m=\frac{n(n+1)}{2}$ locally closed subsets in $X$, namely, the intervals $[a, b]$ for $1 \leq a \leq b \leq n$. For each such $Y$, there is a homological functor $\mathrm{FK}_{Y}: \mathcal{B}(X) \rightarrow \mathfrak{A} \mathfrak{b}^{\mathbb{Z}} / 2$ into the category of $\mathbb{Z} / 2$-graded abelian groups, which computes a certain K-theory corresponding to $Y$. We choose our collection to be

$$
\mathbf{C}_{\mathcal{B}(X)}=\left\{\mathrm{FK}_{Y}\left(-; \mathbb{F}_{p}\right) \mid p \in \operatorname{Spec} \mathbb{Z}, Y \text { is locally closed }\right\},
$$

and define

$$
\operatorname{supp} A:=\left\{(p, Y) \mid \mathrm{FK}_{Y}\left(A ; \mathbb{F}_{p}\right) \neq 0\right\} .
$$

The support of a localizing subcategory is defined to be the union of the supports of its objects. 
This way, the supports of objects in $\mathcal{B}(X)$ live in an $m$-fold cartesian product of power sets of Spec $\mathbb{Z}$. However, unlike in the commutative case, not all elements of this product appear as supports of some localizing subcategory. There is a dependence between functors in $\mathbf{C}_{\mathcal{B}(X)}$ : for any fixed $p \in$ Spec $\mathbb{Z}$ there are exactly $\frac{1}{n+2}\left(\begin{array}{c}2 n+2 \\ n+1\end{array}\right)((n+1)$ th Catalan number $)$ different localizing subcategories with $p$ as a first coordinate in every support point. These subcategories form a lattice isomorphic to the lattice of noncrossing partitions - those partitions of a regular $(n+1)$-gon which do not cross in their planar representation. Summing up, this leads to our main result:

Theorem 6.4. The lattice of all localizing subcategories of $\mathcal{B}(X)$ is isomorphic to the product of lattices of noncrossing partitions of the regular $(n+1)$-gon over the indexing set $\mathrm{Spec} \mathbb{Z}$.

In order to better illustrate what this classification says, consider the example where $X$ has only two points. The category of $\mathrm{C}^{*}$-algebras over $X$ is equivalent to the $\mathfrak{K} \mathfrak{K}$-category of extensions of $\mathrm{C}^{*}$-algebras. Then the theorem classifies all localizing subcategories of the bootstrap class in the $\mathfrak{K} \mathfrak{K}$-category of extensions of $\mathrm{C}^{*}$-algebras in terms of those triples of subsets of Spec $\mathbb{Z}$ which have the property that each one is inside the union of the other two. This is not unexpected, since an earlier result by Alexander Bonkat [8] establishes that isomorphism classes of objects in the bootstrap class of $\mathrm{C}^{*}$-algebra extensions correspond to isomorphism classes of 6-periodic exact chain complexes of countable abelian groups.

This example already reveals how our classification is different from the commutative case. Unfortunately, we cannot hope to recover a space from the lattice of localizing subcategories:

Theorem 5.12. The lattice of localizing subcategories of the bootstrap category $\mathcal{B}(X)$ is not isomorphic to a sublattice of a subset lattice $\mathcal{P}(S)$ for any set $S$.

This already fails for the lattice of noncrossing partitions of the triangle.

\section{The derived category $\mathfrak{D e r}\left(\mathbb{Z} \mathrm{A}_{n} ; \mathbb{Z} / 2\right)_{c}$}

Let $\mathbb{Z} \mathrm{A}_{n}$ denote the ring of upper triangular $n \times n$-matrices with entries in abelian groups. This ring is isomorphic to the path ring of the finite quiver $\mathrm{A}_{n}$, hence the notation.

Let $\mathfrak{C h}\left(\mathbb{Z A} A_{n} ; \mathbb{Z} / 2\right)_{c}$ be the abelian category of 2-periodic chain complexes of countable modules over the ring $\mathbb{Z} \mathrm{A}_{n}$. The 2-periodic derived category $\mathfrak{D e r}\left(\mathbb{Z} \mathrm{A}_{n} ; \mathbb{Z} / 2\right)_{c}$ of countable modules over $\mathbb{Z} \mathrm{A}_{n}$ is defined as the category we get after formally inverting all quasi-isomorphisms in $\mathfrak{C h}\left(\mathbb{Z} \mathrm{A}_{n} ; \mathbb{Z} / 2\right)_{c}$; that is, those maps of chain complexes that induce isomorphisms on all homology groups.

The category $\mathfrak{D e r}(\mathbb{Z A} ; ; \mathbb{Z} / 2)_{c}$ is triangulated [18]. 
Main results for $\mathfrak{D e r}\left(\mathbb{Z} \mathrm{A}_{n} ; \mathbb{Z} / 2\right)_{c}$. The methods developed for $\mathcal{B}(X)$ also apply to $\mathfrak{D} \mathfrak{e r}\left(\mathbb{Z} \mathrm{A}_{n} ; \mathbb{Z} / 2\right)_{c}$. We show that the support theories of $\mathfrak{D e r}\left(\mathbb{Z} \mathrm{A}_{n} ; \mathbb{Z} / 2\right)_{c}$ and $\mathcal{B}(X)$ coincide, by arguing that the collections of functors defining it have similar homological properties. More precisely, we consider the collection of functors

$$
\mathbf{C}_{\mathfrak{D e r}\left(\mathbb{Z} A_{n} ; \mathbb{Z} / 2\right)_{c}}=\left\{\mathrm{FH}_{[a, b]}\left(-; \mathbb{F}_{p}\right) \mid p \in \operatorname{Spec} \mathbb{Z}, 1 \leq a \leq b \leq n\right\},
$$

where each one of them computes a certain homology with coefficients in the residue field $\mathbb{F}_{p}$, and we show that they define the same support theory as the corresponding functors on $\mathcal{B}(X)$.

Hence the lattices of localizing subcategories in these two categories are isomorphic, and we have:

Theorem 7.13. The lattice of all localizing subcategories of $\mathfrak{D e r}\left(\mathbb{Z} \mathrm{A}_{n} ; \mathbb{Z} / 2\right)_{c}$ is isomorphic to the product of lattices of noncrossing partitions of the regular $(n+1)$-gon over the indexing set $\operatorname{Spec} \mathbb{Z}$.

Of course, the negative result of Theorem 5.12 also carries over, telling us that there is no space to be recovered from the ring $\mathbb{Z} \mathrm{A}_{n}$. This is not unexpected, since $\mathbb{Z} \mathrm{A}_{n}$ is a noncommutative ring, and there are some nontrivial obstructions to the existence of a good space-like candidate for its spectrum [35].

It must be noted that similar result to Theorem 7.13 in more general context for the bounded derived category of a finite-dimensional hereditary algebra of finite or tame representation type was obtain with different methods by Claudia Köhler in her dissertation [24]. However, we do not see the straightforward way to carry over her results to the category $\mathfrak{D e r}\left(\mathbb{Z} \mathrm{A}_{n} ; \mathbb{Z} / 2\right)_{c}$.

\section{Outline}

This thesis is organized as follows.

The three preliminary sections in Chapter 2 are written up in a minimalistic manner. We recall the notions directly necessary for our purposes and only prove the facts which cannot be found elsewhere. The first two sections in this chapter are devoted to fixing notation, refreshing the reader's memory about constructions in triangulated categories or providing relevant references. In the third section, we recall the examples of triangulated categories, a concrete case of which will be investigated in the next, contributing chapter.

In Section 4, we prove some general results about localizing subcategories of $\mathcal{B}(X)$, for an arbitrary space $X$. Namely, we show that localizing subcategories are closed under tensoring with $\mathrm{C}^{*}$-algebras, and that they are generated by localization of $\mathrm{C}^{*}$-algebras over $X$ at prime numbers and zero. These results are used in Section 5 to prove the preliminary classification theorem, which classifies localizing subcategories of $\mathcal{B}(X)$ for finite $X$ with totally ordered lattice of open subsets, in terms of certain elements of the $m$-fold cartesian product of power sets of Spec $\mathbb{Z}$. We also discuss the special case of extensions of $\mathrm{C}^{*}$-algebras here, and prove Theorem 5.12. 
In Section 6, we recall the classical definition of the lattice of noncrossing partitions. Then, using the mentioned preliminary theorem, we proceed to prove Theorem 6.4.

In Section 7 , we consider the category $\mathfrak{D e r}\left(\mathbb{Z} \mathrm{A}_{n} ; \mathbb{Z} / 2\right)_{c}$. We collect all necessary facts to conclude that its relative homological algebra is identical to that of $\mathcal{B}(X)$, thus proving Theorem 7.13.

Appendices follow the style of the preliminary sections by only recalling the necessary constructions and providing references. Appendix A.1 recalls the notion of localization for a category, and Appendix A.2 recalls the aspects of quiver representation theory. 


\section{Preliminaries}

\section{Triangulated categories}

The concept of a triangulated category was developed by Jean-Louis Verdier in 1963 (published later in [38]) to axiomatize the structure present in the derived category of an abelian category. Albrecht Dold and Dieter Puppe introduced the same type of axioms when working with the stable homotopy category in the 1961 paper [14]. However, they did not impose the octahedral axiom.

In this section, we recall the definition of a triangulated category and some basic results. It is only supposed to serve as reference for the rest of the thesis; thus the vast majority of the theory is not presented. The relevant examples of triangulated categories will be discussed in Section 2. A good, comprehensive text on the topic is [34].

\subsection{Definition of a triangulated category}

Since we will mainly be dealing with triangulated categories of noncommutative spaces, we will follow the opposite convention on the direction of arrows than usually encountered in the literature. Both approaches are actually equivalent, since a category is triangulated if and only if its opposite is.

Definition 1.1. A stable additive category $\mathfrak{C}$ is an additive category together with an additive autoequivalence functor $\Sigma: \mathfrak{C} \rightarrow \mathfrak{C}$, called suspension.

Definition 1.2. Let $\mathfrak{C}$ be a stable additive category. A triangle in $\mathfrak{C}$ is a diagram of the form

$$
\Sigma B \stackrel{w}{\longrightarrow} C \stackrel{v}{\longrightarrow} A \stackrel{u}{\longrightarrow} B
$$

such that the compositions $v \circ w, u \circ v$ and $w \circ \Sigma u$ are the zero morphisms.

A morphism of triangles is a commutative diagram

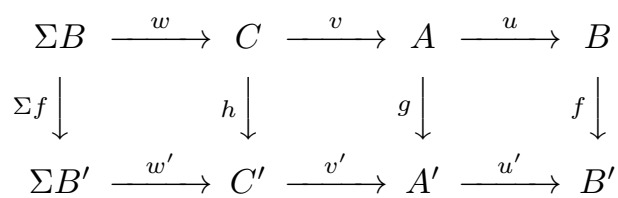

where each row is a triangle.

Definition 1.3. A triangulated category is a stable additive category $\mathfrak{T}$ together with a class of triangles called exact triangles, for which the following conditions are satisfied: 
TR0: The triangle

$$
\Sigma A \rightarrow 0 \rightarrow A \stackrel{\operatorname{id}_{A}}{\longrightarrow} A
$$

is exact and any triangle isomorphic to an exact triangle is exact.

TR1: For any $f \in \mathfrak{T}(A, B)$, there is an object $C_{f} \in \mathfrak{T}$ and an exact triangle

$$
\Sigma B \rightarrow C_{f} \rightarrow A \stackrel{f}{\longrightarrow} B .
$$

(It follows from the other axioms that such a triangle is unique up to noncanonical isomorphism.)

TR2: Consider two triangles

$$
\Sigma B \stackrel{w}{\longrightarrow} C_{u} \stackrel{v}{\longrightarrow} A \stackrel{u}{\longrightarrow} B
$$

and

$$
\Sigma A \stackrel{-\Sigma u}{\longrightarrow} \Sigma B \stackrel{-w}{\longrightarrow} C_{u} \stackrel{-v}{\longrightarrow} A .
$$

If one is exact, then so is the other.

TR3: For any commutative diagram

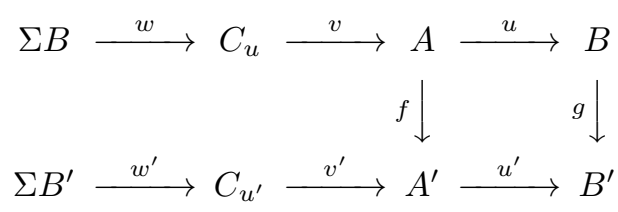

where the rows are exact triangles, there exists a morphism $h: C_{u} \rightarrow C_{u^{\prime}}$, not necessarily unique, which makes the diagram

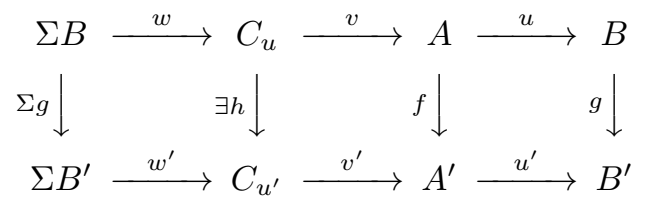

commutative.

TR4 (Octahedral): For any pair of morphisms $f: A \rightarrow B$ and $g: B \rightarrow D$, we have a commutative diagram

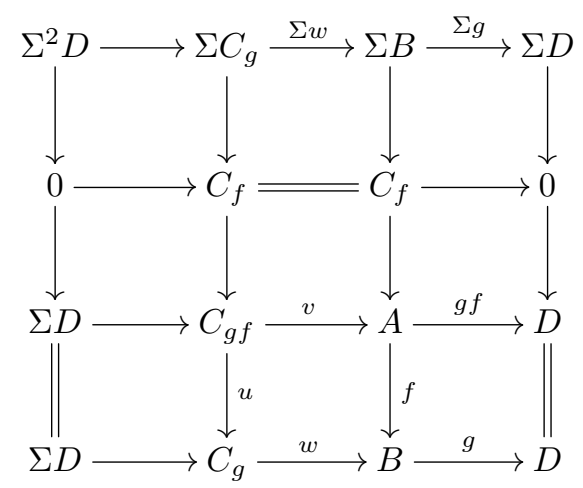


where all rows and columns are exact triangles. Moreover, the triangle

$$
\Sigma B \stackrel{\delta}{\rightarrow} C_{g f} \stackrel{\left(\begin{array}{l}
u \\
v
\end{array}\right)}{\longrightarrow} C_{g} \oplus A \stackrel{(-w f)}{\longrightarrow} B
$$

is also exact, with the map $\delta$ given by the equal composites

$$
\begin{aligned}
& \Sigma B \rightarrow \Sigma D \rightarrow C_{g f} \\
& \Sigma B \rightarrow C_{f} \rightarrow C_{g f} .
\end{aligned}
$$

Definition 1.4. A stable functor $F: \mathfrak{C} \rightarrow \mathfrak{C}^{\prime}$ between two stable additive categories $\left(\mathfrak{C}, \Sigma_{\mathfrak{C}}\right)$ and $\left(\mathfrak{C}^{\prime}, \Sigma_{\mathfrak{C}^{\prime}}\right)$ is an additive functor together with natural isomorphism $F \circ \Sigma_{\mathfrak{C}} \cong \Sigma_{\mathfrak{C}^{\prime}} \circ F$.

Definition 1.5. A stable additive functor between two triangulated categories $F: \mathfrak{T} \rightarrow \mathfrak{U}$ is called exact if it maps exact triangles to exact triangles.

If coproducts of some cardinality exists in the triangulated categories $\mathfrak{T}$ and $\mathfrak{U}$, we also ask an exact functor to preserve this structure. In all examples that are relevant to us, at least countable coproducts exist. Therefore, from now on, we assume the existence of such coproducts in our triangulated categories.

\subsection{Homotopy limits}

In favorable cases, one can construct a good homological substitute for the limit of a diagram in a triangulated category. For example, the object $C_{f}$ with the corresponding map can be considered as a homotopy kernel of a morphism $f \in \mathfrak{T}(A, B)$ in the following sense: By TR0 and TR3, for any map $X \rightarrow A$ such that $X \rightarrow A \stackrel{f}{\rightarrow} B$ is zero, there exists a (not necessarily unique) map $X \rightarrow C_{f}$ such that

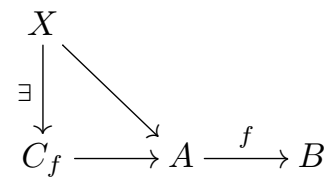

is commutative. Similarly, the object $\Sigma^{-1} C_{f}$ together with the map $B \rightarrow \Sigma^{-1} C_{f}$ can be considered as a homotopy cokernel of $f \in \mathfrak{T}(A, B)$.

Using this analogy, we can construct homotopy pullbacks and pushouts.

Definition 1.6. In a triagulated category $\mathfrak{T}$, a commutative square

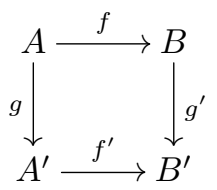

is homotopy cartesian if there is the following exact triangle: 


$$
\Sigma B^{\prime} \rightarrow A \stackrel{\left(\begin{array}{l}
g \\
f
\end{array}\right)}{\longrightarrow} A^{\prime} \oplus B \stackrel{\left(-f^{\prime} g^{\prime}\right)}{\longrightarrow} B^{\prime} .
$$

In this situation, the triple $(A, f, g)$ is called the homotopy pullback of $\left(B^{\prime}, f^{\prime}, g^{\prime}\right)$, and the latter triple is called the homotopy pushout of the former.

Note that the definition is equivalent to saying that $\left(A,\left(\begin{array}{l}g \\ f\end{array}\right)\right)$ is a homotopy kernel of $\left(-f^{\prime} g^{\prime}\right)$, which justifies the terminology.

Next, we define the homotopy version of countable direct limits.

Definition 1.7. Given a countable inductive system $\left(A_{n}, f_{m}^{n}\right)$ in a triangulated category $\mathfrak{T}$, with $f_{m}^{n}: A_{m} \rightarrow A_{n}$ for $m \leq n$, a homotopy direct limit ho-lim $A_{m}$ is the unique object in $\mathfrak{T}$ up to isomorphism that fits in an exact triangle

$$
\Sigma \text { ho- } \longrightarrow \text { lim } A_{m} \rightarrow \bigoplus A_{m} \stackrel{\text { id-shift }_{f}}{\longrightarrow} \bigoplus A_{m} \rightarrow \text { ho-lim } A_{m}
$$

where $\operatorname{shift}_{f}$ is the map that maps the summand $A_{m}$ to $A_{m+1}$ via $f_{m}^{m+1}$.

\subsection{Localization of a triangulated category}

Localization theory of triangulated categories is a large subject. Our exposition only recalls the facts that are used later for new results. Here we present Verdier localization. The following facts are proven in [34].

Definition 1.8. Let $\mathfrak{T}$ be a triangulated category. A full additive subcategory $\mathfrak{S}$ in $\mathfrak{T}$ is called a triangulated subcategory if every object isomorphic to an object of $\mathfrak{S}$ is in $\mathfrak{S}$, if $\Sigma \mathfrak{S}=\mathfrak{S}$, and if for any exact triangle

$$
\Sigma B \rightarrow C \rightarrow A \rightarrow B
$$

such that the objects $A$ and $B$ are in $\mathfrak{S}$, the object $C$ is also in $\mathfrak{S}$.

Remark 1.9. Let $\mathfrak{S}$ be a triangulated subcategory in $\mathfrak{T}$ and let

$$
\Sigma B \rightarrow C \rightarrow A \rightarrow B
$$

be an exact triangle in $\mathfrak{T}$. If any two of the objects $A, B$ or $C$ are in $\mathfrak{S}$, then so is the third. This is true because we can rotate exact triangles by TR2.

Definition 1.10. A triangulated subcategory $\mathfrak{S} \subseteq \mathfrak{T}$ is localizing if it is closed under formation of whatever coproducts exist in $\mathfrak{T}$.

For any family of objects $\left\{A_{\alpha} \mid \alpha \in I\right\}$ in $\mathfrak{T}$, the smallest localizing subcategory that contains all $A_{\alpha}$ is denoted by $\left\langle A_{\alpha} \mid \alpha \in I\right\rangle$.

Remark 1.11. In the literature, localizing subcategories are defined as triangulated subcategories which, together with being closed under coproducts, are also closed under formation of retracts (are thick). However, since all our triangulated categories have at least countable coproducts, closure under taking retracts follows from the other properties. 
1. Triangulated categories

For an exact functor $F: \mathfrak{T} \rightarrow \mathfrak{U}$ between triangulated categories, denote by $\operatorname{ker}_{o} F$ the full subcategory of $\mathfrak{T}$ whose objects map to objects of $\mathfrak{U}$ isomorphic to 0 (we used the subscript " $O$ " for "objects", in order to distinguish ker $_{o} F$ from the kernel on morphisms used in the following chapters). Then it is easy to see that $\operatorname{ker}_{o} F$ is a triangulated subcategory of $\mathfrak{T}$ ([34, Lemma 2.1.4]), and it is localizing because, by convention, exact functors commute with coproducts.

The following theorem by Verdier [38] describes the role of triangulated subcategories:

Theorem 1.12. For any triangulated subcategory $\mathfrak{S} \subset \mathfrak{T}$, there exists a triangulated category $\mathfrak{T} / \mathfrak{S}$ and an exact functor $Q: \mathfrak{T} \rightarrow \mathfrak{T} / \mathfrak{S}$, so that $\mathfrak{S} \subseteq \operatorname{ker}_{o} Q$, and any exact functor $F: \mathfrak{T} \rightarrow \mathfrak{T}^{\prime}$ whose kernel contains $\mathfrak{S}$ factors uniquely as

$$
\mathfrak{T} \stackrel{Q}{\rightarrow} \mathfrak{T} / \mathfrak{S} \rightarrow \mathfrak{T}^{\prime}
$$

It follows form the axioms of triangulated categories that a morphism $Q(A) \stackrel{Q(f)}{\longrightarrow} Q(B)$ is an isomorphism if and only if $Q\left(C_{f}\right) \cong 0$, that is, its homotopy kernel vanishes. Hence $\mathfrak{T} / \mathfrak{S}$ is a localization of $\mathfrak{T}$ with respect to morphisms whose homotopy kernels vanish under $Q$ (for the definition of the localization of a general category and the calculus of fractions, see Appendix A.1). In particular, all morphisms $f: A \rightarrow B$ with $C_{f} \in \mathfrak{S}$ are inverted. Denote this class of morphisms by Mor . .

Proposition 1.13. For any triangulated subcategory $\mathfrak{S} \subseteq \mathfrak{T}$ in a triangulated category, Mor $_{\mathfrak{S}}$ admits a calculus of fractions.

If $\mathfrak{S}$ is closed under coproducts then $\mathfrak{S} \cong \operatorname{ker}_{o} Q$. So when one wishes to study all possible localizations of a triangulated category with coproducts, one only needs to consider localizing subcategories.

\subsection{Homological functors}

Now we define homology theories for triangulated categories. Homological invariants are crucial in our approach.

Definition 1.14. An additive functor $H: \mathfrak{T} \rightarrow \mathcal{A}$ from a triangulated category $\mathfrak{T}$ to an abelian category $\mathfrak{A}$ is called homological if, for every exact triangle

$$
\Sigma B \stackrel{w}{\longrightarrow} C \stackrel{v}{\longrightarrow} A \stackrel{u}{\longrightarrow} B,
$$

the sequence

$$
H(C) \stackrel{H(v)}{\longrightarrow} H(A) \stackrel{H(u)}{\longrightarrow} H(B)
$$

is exact in $\mathfrak{A}$.

Since we can rotate triangles by TR2, the exact sequence above can be continued indefinitely in both directions. In other words, we have a long exact sequence

$\cdots \rightarrow H(\Sigma B) \stackrel{H(w)}{\longrightarrow} H(C) \stackrel{H(v)}{\longrightarrow} H(A) \stackrel{H(u)}{\longrightarrow} H(B) \stackrel{H\left(\Sigma^{-1} w\right)}{\longrightarrow} H\left(\Sigma^{-1} C\right) \rightarrow \cdots$ 
Lemma 1.15. Let $\mathfrak{T}$ be a triangulated category, let $D$ be an object in $\mathfrak{T}$. Then the representable functor $\mathfrak{T}(D,-)$ is homological.

\section{Homological algebra in triangulated categories}

In this section we briefly discuss the relevant aspects of the theory of homological algebra in a triangulated category.

If the category in question is not abelian, homological algebra is always relative, that is, additional data is necessary to get started. In the case of triangulated categories, the theory turns out to be remarkably rich, as demonstrated when the subject was first explored in the works of J. Daniel Christensen [9] and Apostolos Beligiannis [4].

The relative homological algebra in the context of triangulated categories of our interest was developed by Ralf Meyer and Ryszard Nest in [31, 28]. All the definitions and proofs of the facts stated here can be found in [31].

\section{$2.1 \quad$ Ideals}

For additional structure needed for homological algebra we use the notion of ideals.

Definition 2.1. An ideal $\mathfrak{I}$ in a triangulated category $\mathfrak{T}$ is a collection of subgroups $\mathfrak{I}(A, B) \subseteq \mathfrak{T}(A, B)$ for all pairs of objects $A, B \in \mathfrak{T}$, such that

$$
\mathfrak{T}(C, D) \circ \mathfrak{I}(B, C) \circ \mathfrak{T}(A, B) \subseteq \mathfrak{I}(A, D)
$$

for all $A, B, C, D \in \mathfrak{T}$.

For example, any homological functor $F: \mathfrak{T} \rightarrow \mathfrak{A}$ into an abelian category $\mathfrak{A}$ defines the kernel ideal ker $F$ by

$$
\operatorname{ker} F(A, B):=\{f \in \mathfrak{T}(A, B) \mid F(f)=0\} .
$$

Definition 2.2. An ideal $\mathfrak{I}$ in a triangulated category $\mathfrak{T}$ is called homological if it is the kernel of a stable homological functor.

Different functors can give rise to the same homological ideal. However, the resulting homological algebra only depends on the ideal itself.

The morphisms in $\mathfrak{I}$ should be thought of as being zero "up to" $\mathfrak{I}$ in $\mathfrak{T}$. This intuition justifies the following terminology

Definition 2.3. Given an exact triangle

$$
\Sigma B \stackrel{w}{\longrightarrow} C \stackrel{v}{\longrightarrow} A \stackrel{u}{\longrightarrow} B
$$

in $\mathfrak{T}$, we say that it is $\mathfrak{I}$-exact if $w \in \mathfrak{I}(\Sigma B, C)$. Moreover, in this situation, we say that $u$ is $\mathfrak{I}$-epic, $v$ is $\mathfrak{I}$-monic and $w$ is $\mathfrak{I}$-phantom.

We will only be dealing with homological ideals. 
Definition 2.4. For a homological ideal $\mathfrak{I}=\operatorname{ker} F$, a chain complex $C \bullet$ $\left(C_{n}, d_{n}\right)$ over $\mathfrak{T}$ is $\mathfrak{I}$-exact in degree $n$ if

$$
F\left(C_{n+1}\right) \stackrel{F\left(d_{n+1}\right)}{\longrightarrow} F\left(C_{n}\right) \stackrel{F\left(d_{n}\right)}{\longrightarrow} F\left(C_{n-1}\right)
$$

is exact at $F\left(C_{n}\right)$.

We call $C \bullet \mathfrak{I}$-exact if it is $\mathfrak{I}$-exact in degree $n$ for all $n \in \mathbb{Z}$.

\subsection{Relative projective objects and exactness}

Definition 2.5. A homological functor $F: \mathfrak{T} \rightarrow \mathfrak{A}$ into some abelian category $\mathfrak{A}$ is $\mathfrak{I}$-exact if $F(f)=0$ for all $A, B \in \mathfrak{T}$ and $f \in \mathfrak{I}(A, B)$, that is, $\mathfrak{I} \subseteq \operatorname{ker} F$.

Definition 2.6. An object $A \in \mathfrak{T}$ is $\mathfrak{I}$-projective if the functor $\mathfrak{T}(A,-)$ is I-exact.

Denote the full subcategory of $\mathfrak{I}$-projective objects by $\mathfrak{P}_{\mathfrak{J}}$. Clearly, $\mathfrak{P}_{\mathfrak{I}}$ is closed under forming retracts (taking direct summands), (de)suspensions and whatever coproducts exist in $\mathfrak{T}$.

Definition 2.7. Let $\mathfrak{I}$ be a homological ideal in $\mathfrak{T}$ and $A \in \mathfrak{T}$. We say that $\pi: P \rightarrow A$ is a one-step $\mathfrak{I}$-projective resolution if $\pi$ is $\mathfrak{I}$-epic and $P \in \mathfrak{P}_{\mathfrak{I}}$. An $\mathfrak{I}$-projective resolution of $A$ is an $\mathfrak{I}$-exact chain complex

$$
\cdots \rightarrow P_{n} \rightarrow P_{n-1} \rightarrow \cdots \rightarrow P_{0} \rightarrow A
$$

with $P_{n} \in \mathfrak{P}_{\mathfrak{I}}$ for all $n \in \mathbb{N}$.

We say that there are enough $\mathfrak{I}$-projective objects in $\mathfrak{T}$ if every object $A \in \mathfrak{T}$ has a one-step projective resolution.

The following proposition shows that relative projective objects have properties similar to projective objects in an abelian category.

Proposition 2.8 (Meyer-Nest [31, Proposition 3.26]). Every object in $\mathfrak{T}$ has an $\mathfrak{I}$-projective resolution if and only if $\mathfrak{T}$ has enough $\mathfrak{I}$-projective objects.

Any map between objects of $\mathfrak{T}$ can be lifted to a chain map between $\mathfrak{I}$-projective resolutions of these objects, and this lifting is unique up to chain homotopy. Two I-projective resolutions of the same object are chain homotopy equivalent.

\subsection{The universal I-exact functor}

For any triangulated cateogry $\mathfrak{T}$, there exists a universal stable homological functor $U: \mathfrak{T} \rightarrow \mathcal{A}(\mathfrak{T})$ into a certain stable abelian category $\mathcal{A}(\mathfrak{T})$ such that any other stable homological functor from $\mathfrak{T}$ into a stable abelian category $\mathfrak{A}$ factors as $\mathfrak{T} \rightarrow \mathcal{A}(\mathfrak{T}) \stackrel{\exists !}{\rightarrow} \mathfrak{A}$ for a stable exact functor $\mathcal{A}(\mathfrak{T}) \rightarrow \mathfrak{A}$ which is unique up to canonical isomorphism. This is a classical construction by Peter Freyd [16].

As it turns out, we can construct a relative version of these statements: 
Definition 2.9. Let $\mathfrak{I}$ be a homological ideal in a triangulated category $\mathfrak{T}$. Then an $\mathfrak{I}$-exact stable homological functor $F$ is universal, if any other $\mathfrak{I}$-exact stable homological functor $G: \mathfrak{T} \rightarrow \mathfrak{A}^{\prime}$ factors as $G=\bar{G} \circ F$ for a stable exact functor $\bar{G}: \mathfrak{A} \rightarrow \mathfrak{A}^{\prime}$ that is unique up to natural isomorphism.

Theorem 2.10 (Beligiannis [4, Section 3]). For every homological ideal $\mathfrak{I}$ in a triangulated category $\mathfrak{T}$, there exists an abelian category $\mathcal{A}_{\mathfrak{T}}(\mathfrak{T})$ and a universal $\mathfrak{I}$-exact stable homological functor $F: \mathfrak{T} \rightarrow \mathcal{A}_{\mathfrak{I}}(\mathfrak{T})$.

Here the category $\mathcal{A}_{\mathfrak{I}}(\mathfrak{T})$ is obtained by localizing the Freyd category $\mathcal{A}(\mathfrak{T})$ at an appropriate Serre subcategory, where we quotient out all morphisms coming from the ideal $\mathfrak{I}$. There may be some set theory issues here with sets and classes. $\mathcal{A}(\mathfrak{T})$ and $\mathcal{A}_{\mathfrak{I}}(\mathfrak{T})$ may become "large" categories.

Roughly speaking, having a universal I-exact stable homological functor means that homological algebra in the target abelian category is the same as in the domain triangulated category with respect to the ideal $\mathfrak{I}$.

Theorem 2.11 (Beligiannis [4, Proposition 4.19]). Let $\mathfrak{I}$ be a homological ideal in a triangulated category $\mathfrak{T}$ and let $F: \mathfrak{T} \rightarrow \mathfrak{A}$ be a universal $\mathfrak{I}$-exact stable homological functor into a stable abelian category $\mathfrak{A}$. Suppose that idempotent morphisms in $\mathfrak{T}$ split and that there are enough $\mathfrak{I}$-projective objects in $\mathfrak{T}$. Then there are enough projective objects in $\mathfrak{A}$ and $F$ induces an equivalence between the full subcategories of $\mathfrak{I}$-projective objects in $\mathfrak{T}$ and of projective objects in $\mathfrak{A}$.

It is also possible to define derived functors relative to a homological ideal $\mathfrak{I}$. There is a spectral sequence that relates a homological functor to its derived functors. We are not going to discuss this general construction. We are only going to recall the favorable case of a Universal Coefficient Theorem, where this spectral sequence degenerates to a short exact sequence and we are able to compute the derived functors using the universal $\mathfrak{I}$-exact functor.

Theorem 2.12 (Meyer-Nest [31, Theorem 4.4]). Let $\mathfrak{I}$ be a homological ideal in a triangulated category $\mathfrak{T}$ and let $F: \mathfrak{T} \rightarrow \mathfrak{A}$ be a universal $\mathfrak{I}$-exact stable homological functor into a stable abelian category $\mathfrak{A}$ with enough projective objects. For $A \in \mathfrak{T}$, let $F(A)$ have a projective resolution of length 1. Suppose also that $A \in\left\langle\mathfrak{P}_{\mathfrak{I}}\right\rangle$. Then for any $B \in \mathfrak{T}$ there is a natural short exact sequence

$$
\operatorname{Ext}_{\mathfrak{A}}^{1}(F(\Sigma A), F(B)) \hookrightarrow \mathfrak{T}(A, B) \rightarrow \operatorname{Hom}_{\mathfrak{A}}(F(A), F(B)),
$$

where $\operatorname{Ext}_{\mathfrak{A}}^{1}$ and $\operatorname{Hom}_{\mathfrak{A}}$ denote extension and morphism groups in $\mathfrak{A}$ and $\Sigma$ is a suspension on $\mathfrak{T}$.

\section{Relevant examples}

In this section, we will take a look at three examples of triangulated categories: the derived category of an abelian category, Kasparov's $\mathfrak{K} \mathfrak{K}$-category for $\mathrm{C}^{*}$ algebras and Kasparov's $\mathfrak{K} \mathfrak{K}$-category for $\mathrm{C}^{*}$-algebras with the action of a topological space. We will not try to extensively motivate the study of these 
3. Relevant examples

categories because there are plenty of very good references that do so. For Kasparov categories, see, for example, [29, 19] and [30]; for the derived category, see [38] or [23]. The notions and facts recalled here will be used later in Chapter 3.

\subsection{The derived category of an abelian category}

As already mentioned, it was the study of the derived category that led JeanLouis Verdier to the notion of a triangulated category.

The derived category of an abelian category $\mathfrak{A}$ is the natural home for doing any kind of homological algebra on $\mathfrak{A}$. Notions like projective resolutions and derived functors naturally manifest themselves on the derived category.

All the definitions and proofs of the facts recalled here, unless stated otherwise, can be found in classical sources like [18] or [39].

Definition 3.1. Let $\mathfrak{A}$ be an abelian category and $m \in \mathbb{N}$. Let $\mathfrak{C h}(\mathfrak{A} ; \mathbb{Z} / m)$ be the category of unbounded m-periodic chain complexes over $\mathfrak{A}$. More precisely, objects $\left(A^{\bullet}, d_{A}^{\bullet}\right) \in \mathfrak{C h}(\mathfrak{A} ; \mathbb{Z} / m)$ satisfy $A^{n}=A^{n+m}$ and $d^{n}=(-1)^{m} d^{n+m}$ for all $n \in \mathbb{Z}$, and chain maps are $m$-periodic.

For $m=0$ we recover the usual non-periodic category of unbounded chain complexes. By convention, we write $\mathbb{Z} / 0:=\mathbb{Z}$.

$\mathfrak{C h}(\mathfrak{A} ; \mathbb{Z} / m)$ is also an abelian category.

Definition 3.2. A homotopy in $\mathfrak{C} \mathfrak{h}(\mathfrak{A} ; \mathbb{Z} / m)$ is a chain homotopy between chain maps which is $m$-periodic, that is, $h^{n+m}=(-1)^{m} h^{n}$.

Definition 3.3. Let $\mathfrak{H} \mathfrak{o}(\mathfrak{A} ; \mathbb{Z} / m)$ be the homotopy category of $\mathfrak{C h}(\mathfrak{A} ; \mathbb{Z} / m)$. It has same the objects, and the group of morphisms $A \rightarrow A^{\prime}$ in $\mathfrak{H} \mathfrak{o}(\mathfrak{A} ; \mathbb{Z} / m)$ is the group $\left[A, A^{\prime}\right]$ of homotopy classes of chain maps from $A$ to $A^{\prime}$.

The category $\mathfrak{H o}(\mathfrak{A} ; \mathbb{Z} / m)$ is already triangulated. However, the convention on the direction of arrows is opposite to the one we used to axiomatize this structure. As mentioned earlier, this makes no difference, so we ignore the issue.

Definition 3.4. Let $f: A \rightarrow B$ be a map of chain complexes in $\mathfrak{H o}(\mathfrak{A} ; \mathbb{Z} / m)$. Define the cone of $f$ to be the chain complex $\operatorname{cone}(f)=A[1] \oplus B$ with differential

$$
d_{\text {cone }(f)}=\left(\begin{array}{cc}
d_{A[1]} & 0 \\
f[1] & d_{B}
\end{array}\right),
$$

where $A[1]$ denotes the chain complex with $A[1]^{n}=A^{n+1}, d_{A[1]}^{n}=-d_{A}^{n+1}$, and $f[1]^{n}=f^{n+1}$.

For every morphism $f: A \rightarrow B$ in $\mathfrak{H} \mathfrak{o}(\mathfrak{A} ; \mathbb{Z} / m)$, the obvious projection maps give the mapping cone triangle

$$
A \rightarrow B \rightarrow \operatorname{cone}(f) \rightarrow A[1] .
$$


Call a triangle in $\mathfrak{H} \mathfrak{o}(\mathfrak{A} ; \mathbb{Z} / m)$ exact if it is isomorphic to a mapping cone triangle. The additive category $\mathfrak{H} \mathfrak{o}(\mathfrak{A} ; \mathbb{Z} / m)$ together with the automorphism [1] and the class of exact triangles is a triangulated category.

Of course, the homology of an $m$-periodic chain complex in $\mathfrak{H} \mathfrak{o}(\mathfrak{A} ; \mathbb{Z} / m)$ is also $m$-periodic. So we get a homology functor

$$
\mathrm{H}_{*}: \mathfrak{H o}(\mathfrak{A} ; \mathbb{Z} / m) \rightarrow \mathfrak{A}^{\mathbb{Z} / m},
$$

where $\mathfrak{A}^{\mathbb{Z} / m}$ is the category of $\mathbb{Z} / m$-graded objects of $\mathfrak{A}$. This functor is clearly stable with respect to a shift automorphism on both categories.

Definition 3.5. Let $\mathfrak{N}(\mathfrak{A} ; \mathbb{Z} / m) \subset \mathfrak{H} \mathfrak{o}(\mathfrak{A} ; \mathbb{Z} / m)$ be the full subcategory of those chain complexes $A \in \mathfrak{H} \mathfrak{o}(\mathfrak{A} ; \mathbb{Z} / m)$ whose homology vanishes in every degree, $\mathrm{H}_{*}(A) \cong 0$; or equivalently $\mathfrak{N}(\mathfrak{A} ; \mathbb{Z} / m):=\operatorname{ker}_{o} \mathrm{H}_{*}$.

As an object kernel of a stable homological functor, $\mathfrak{N}(\mathfrak{A} ; \mathbb{Z} / m)$ is easily seen to be a localizing subcategory of $\mathfrak{H o}(\mathfrak{A} ; \mathbb{Z} / m)$. The objects in $\mathfrak{N}(\mathfrak{A} ; \mathbb{Z} / m)$ are called acyclic.

Definition 3.6. The $m$-periodic derived category $\mathfrak{D e r}(\mathfrak{A} ; \mathbb{Z} / m)$ of an abelian category $\mathfrak{A}$ is the localization of $\mathfrak{H o}(\mathfrak{A} ; \mathbb{Z} / m)$ at the localizing subcategory $\mathfrak{N}(\mathfrak{A} ; \mathbb{Z} / m)$. That is,

$$
\mathfrak{D e r}(\mathfrak{A} ; \mathbb{Z} / m)=\mathfrak{H} \mathfrak{o}(\mathfrak{A} ; \mathbb{Z} / m) / \mathfrak{N}(\mathfrak{A} ; \mathbb{Z} / m) .
$$

In other words, we arrive at $\mathfrak{D e r}(\mathfrak{A} ; \mathbb{Z} / m)$ by inverting all arrows $f: A \rightarrow B$ in $\mathfrak{H o}(\mathfrak{A} ; \mathbb{Z} / m)$ such that their cone is acyclic, $\mathrm{H}_{*}(\operatorname{cone}(f)) \cong 0$. Since $\mathrm{H}_{*}$ is homological, such morphisms are exactly the ones that induce isomorphism on homology.

Definition 3.7. A quasi-isomorphism is a morphism in $\mathfrak{H} \mathfrak{o}(\mathfrak{A} ; \mathbb{Z} / m)$ that induces an isomorphism on homology.

Hence by Proposition 1.13 quasi-isomorphisms have a calculus of fractions. As recalled in Appendix A.1, then every morphism $f: A \rightarrow B$ in $\mathfrak{D e r}(\mathfrak{A} ; \mathbb{Z} / m)$ is of the form $A \stackrel{s}{\leftarrow} X \stackrel{g}{\rightarrow} B$ for a quasi-isomorphism $s$, a morphism $g$, and an object $X$, all in $\mathfrak{H} \mathfrak{o}(\mathfrak{A} ; \mathbb{Z} / m)$.

In the case $m=0$, for the construction of the category $\mathfrak{D} \mathfrak{e r}(\mathfrak{A}):=\mathfrak{D} \mathfrak{e r}(\mathfrak{A}, \mathbb{Z})$, we could have only considered the chain complexes that are bounded, that is, the chain complexes with only finitely many nonzero terms. This gives the derived category $\mathfrak{D e r}^{b}(\mathfrak{A}) \subset \mathfrak{D e r}(\mathfrak{A})$ of bounded chain complexes over $\mathfrak{A}$.

\section{The derived category of a ring}

Let the abelian category in question be the category of modules $\mathfrak{M o d}(R)$ over a ring $R$. Then for simplicity we denote $\mathfrak{D e r}(R ; \mathbb{Z} / m):=\mathfrak{D e r}(\mathfrak{M o d}(R) ; \mathbb{Z} / m)$ for $m \in \mathbb{N}$.

We recall some classical results for the derived category of a ring. For $i \in \mathbb{Z} / m$, denote by $R[i]$ the chain complex with the module $R$ in the place $i$ and zeros elsewhere. 
Proposition 3.8. The homology functors are representable. More precisely, the Yoneda natural transformation corresponding to the unit $1_{R} \in R[i]$ is an isomorphism of functors

$$
\mathfrak{D e r}(R ; \mathbb{Z} / m)(R[i],-) \cong \mathrm{H}_{i}(-)
$$

for all $i \in \mathbb{Z} / m$.

The total homology functor $\mathrm{H}_{*}$ is represented by the direct sum $\bigoplus_{i \in \mathbb{Z} / m} R[i]$.

Hence $\mathfrak{D e r}(R ; \mathbb{Z} / m)$ is generated by the objects $\{R[i] \mid i \in \mathbb{Z} / m\}$ :

Proposition 3.9. The smallest localizing subcategory in $\mathfrak{D e r}(R ; \mathbb{Z} / m)$ containing the objects $\{R[i] \mid i \in \mathbb{Z} / m\}$ is the whole $\mathfrak{D e r}(R ; \mathbb{Z} / m)$. In other words,

$$
\langle R[i] \mid i \in \mathbb{Z} / m\rangle \cong \mathfrak{D e r}(R ; \mathbb{Z} / m) .
$$

\section{Derived tensor product}

We will also use the tensor structure present on the derived category of a ring. The facts recalled here can be found in [25].

For a commutative ring $R$, denote $\mathfrak{C h}(R ; \mathbb{Z} / m)=\mathfrak{C h}(\mathfrak{M o d}(R) ; \mathbb{Z} / m)$.

Definition 3.10. For complexes $A, B \in \mathfrak{C h}(R ; \mathbb{Z} / m)$, their tensor product $A \otimes_{R} B$ is the chain complex whose component in degree $n$ is given by

$$
\left(A \otimes_{R} B\right)^{n}=\bigoplus_{i+j=n} A^{i} \otimes_{R} B^{j}
$$

and whose differential is

$$
d_{A \otimes_{R} B}^{n}\left(a^{i} \otimes b^{j}\right)=\left(d_{A}\left(a^{i}\right), b^{j}\right)+(-1)^{i}\left(a^{i}, d_{B}\left(b^{j}\right)\right)
$$

for $a^{i} \otimes b^{j} \in A^{i} \otimes_{R} B^{j}$ with $i+j=n$.

The tensor product of chain complexes descends to a monoidal structure on the derived category.

Definition 3.11. A chain complex $F \in \mathfrak{C h}(R ; \mathbb{Z} / m)$ is called flat if for every acyclic complex $A \in \mathfrak{C h}(R ; \mathbb{Z} / m)$ the tensor product $A \otimes_{R} F$ is also acyclic.

Tensoring with a flat complex $F$ descends to an exact functor

$$
\mathfrak{H} \mathfrak{o}(R ; \mathbb{Z} / m) \stackrel{-\otimes_{R} F}{\longrightarrow} \mathfrak{H o}(R ; \mathbb{Z} / m)
$$

that transforms quasi-isomorphisms into quasi-isomorphisms because tensoring is an exact functor and quasi-isomorphisms in $\mathfrak{H o}(R ; \mathbb{Z} / m)$ are characterized by having acyclic cones.

Lemma 3.12. For any complex $A \in \mathfrak{D e r}(R ; \mathbb{Z} / m)$ there exists a flat complex $F \in \mathfrak{D e r}(R ; \mathbb{Z} / m)$ and a quasi-isomorphism $F \rightarrow A$ called a flat resolution. 
Picking a flat resolution for every complex gives an exact bifunctor

$$
-\otimes_{R}-: \mathfrak{D e r}(R ; \mathbb{Z} / m) \times \mathfrak{D e r}(R ; \mathbb{Z} / m) \rightarrow \mathfrak{D e r}(R ; \mathbb{Z} / m),
$$

because $\mathfrak{D e r}(R ; \mathbb{Z} / m)$ is the localization of $\mathfrak{H o}(R ; \mathbb{Z} / m)$ at quasi-isomorphisms. Also, tensoring two quasi-isomorphic flat complexes gives quasi-isomorphic tensor products, so this functor does not depend on the choice of a flat resolution.

If the ring $R$ is noncommutative, we can still tensor (right) $R$-modules with abelian groups and get back $R$-modules. In other words, the same construction gives an exact bifunctor

$$
-\otimes_{\mathbb{Z}}-: \mathfrak{D e r}(\mathbb{Z} ; \mathbb{Z} / m) \times \mathfrak{D e r}(R ; \mathbb{Z} / m) \rightarrow \mathfrak{D e r}(R ; \mathbb{Z} / m)
$$

\subsection{Bivariant K-theory}

Bivariant K-theory is a joint generalization of topological K-theory and its dual, K-homology. Seen as a category, it is a universal home for split-exact, compact-stable and homotopy-invariant functors on the category of $\mathrm{C}^{*}$-algebras. Therefore, bivariant K-theory plays a fundamental role in noncommutative topology, noncommutative geometry and index theory.

We assume that the reader is familiar with the definition and the basic properties of the category of $\mathrm{C}^{*}$-algebras (see, for instance, [11] and [27]). All facts mentioned in this section can be found in [7].

For future reference, we recall the definition of Kasparov cycles:

Definition 3.13. Let $A, B$ be separable $\mathrm{C}^{*}$-algebras.

- An even or odd Kasparov cycle between $A$ and $B$ is a a triple $\left(\varphi, \mathcal{H}_{B}, F\right)$, where

- $\mathcal{H}_{B}$ is a right Hilbert $B$-module. In the even case, we assume the existence of a $\mathbb{Z} / 2$-grading on $\mathcal{H}_{B}$;

- $F \in \mathbb{B}\left(\mathcal{H}_{B}\right)$ is an adjointable operator on $\mathcal{H}_{B}$ which commutes with the grading in the even case;

$-\varphi: A \rightarrow \mathbb{B}\left(\mathcal{H}_{B}\right)$ is a ${ }^{*}$-representation commuting with the grading in the even case;

- $\varphi(a)\left(F-F^{*}\right), \varphi(a)\left(F^{2}-1\right)$ and the commutator $[\varphi(a), F]$ are compact for every $a \in A$;

- Two cycles $\left(\varphi^{0}, \mathcal{H}_{B}^{0}, F^{0}\right)$ and $\left(\varphi^{1}, \mathcal{H}_{B}^{1}, F^{1}\right)$ are unitarily equivalent if there is a unitary $\mathcal{H}_{B}^{0} \rightarrow \mathcal{H}_{B}^{1}$ (of degree 0 in the even case) intertwining $\varphi_{i}$ and $F_{i}$ for $i=0,1$.

- Two cycles $\left(\varphi^{0}, \mathcal{H}_{B}^{0}, F^{0}\right)$ and $\left(\varphi^{1}, \mathcal{H}_{B}^{1}, F^{1}\right)$ between $\mathrm{C}^{*}$-algebras $A$ and $B$ are said to be homotopic if there is a cycle $\left(\varphi, \mathcal{H}_{I B}, F\right)$ between $A$ and $I B:=\mathrm{C}([0,1], B)$ such that $\left(\mathrm{ev}_{i} \circ \varphi, \mathcal{H}_{B I} \otimes_{\mathrm{ev}_{i}} B, \mathrm{ev}_{i *}(F)\right)$ is unitarily equivalent to $\left(\varphi^{i}, \mathcal{H}_{B}^{i}, F^{i}\right)$ for $i=0,1$, where the maps ev $i$ are the evaluation homomorphisms from $I B$ to $B$. 
This gives a $\mathbb{Z} / 2$-graded abelian group of homotopy classes of cycles from $A$ to $B$ with addition given by direct sum of Kasparov cycles. Denote this group by $\mathrm{KK}_{*}(A, B)$.

The groups $\operatorname{KK}_{*}(A, B)$ define a bifunctor, covariant in the first and contravariant in the second variable, from the category of separable $\mathrm{C}^{*}$-algebras to $\mathbb{Z} / 2$-graded abelian groups.

One of the remarkable features of Kasparov theory is the existence of a natural, associative product

$$
\mathrm{KK}_{i}(A, B) \times \mathrm{KK}_{j}(B, C) \rightarrow \mathrm{KK}_{i+j}(A, C) .
$$

This allows us to define

Definition 3.14. Let $\mathfrak{K} \mathfrak{K}$ be the category with objects separable $\mathrm{C}^{*}$-algebras and with morphism sets $\operatorname{KK}_{0}(A, B)$ for $A, B \in \mathfrak{K} \mathfrak{K}$.

The category $\mathfrak{K} \mathfrak{K}$ is additive with countable coproducts given by $\mathrm{C}_{0}$-direct sums of $\mathrm{C}^{*}$-algebras. The tensor product of $\mathrm{C}^{*}$-algebras (either minimal or maximal) induces a monoidal structure on $\mathfrak{K} \mathfrak{K}$. As a result, since the bifunctor $\mathrm{KK}_{*}(-,-)$ is homotopy invariant and satisfies Bott periodicity, $\mathfrak{K} \mathfrak{K}$ is a stable category. Here stability is with respect to the suspension functor

$$
\Sigma: \mathfrak{K} \mathfrak{K} \rightarrow \mathfrak{K} \mathfrak{K} \quad A \mapsto \mathrm{C}_{0}(\mathbb{R}) \otimes A,
$$

where there is no ambiguity because $\mathrm{C}_{0}(\mathbb{R}) \otimes_{\min } A \cong \mathrm{C}_{0}(\mathbb{R}) \otimes_{\max } A$.

\section{Triangulated structure of $\mathfrak{K} \mathfrak{K}$}

The facts recalled in this section can be found in [29].

We already mentioned that $\mathfrak{K} \mathfrak{K}$ is additive. Also, by Bott periodicity, $\Sigma$ is an automorphism (up to natural isomorphism).

Let $I \hookrightarrow E \rightarrow Q$ be an extension of $\mathrm{C}^{*}$-algebras. It is called a split extension if it splits by a ${ }^{*}$-homomorphism. It is called semi-split if there is a completely positive, contractive section $Q \rightarrow E$.

Recall that the cone of a map $A \stackrel{f}{\rightarrow} B$ between $\mathrm{C}^{*}$-algebras is defined as cone $(f):=\left\{(a, b) \in A \times \mathrm{C}_{0}((0,1], B) \mid f(a)=b(1)\right\}$.

For every semi-split extension $I \hookrightarrow E \rightarrow Q$, where $I, E, Q$ are separable $\mathrm{C}^{*}$-algebras, there exists a unique map $\Sigma Q \rightarrow I$ and an isomorphism $I \stackrel{\cong}{\rightarrow}$ $\operatorname{cone}(E \rightarrow Q)$, both in $\mathfrak{K} \mathfrak{K}$, such that the following diagram is an isomorphism of triangles:

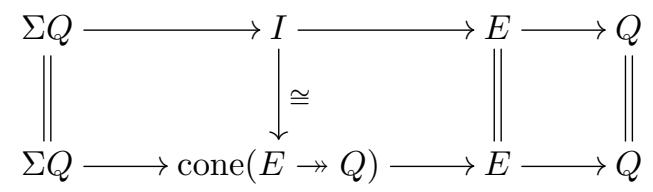

The first triangle in the diagram is called the extension triangle of the semi-split extension $I \hookrightarrow E \rightarrow Q$. 
Declare all triangles in $\mathfrak{K} \mathfrak{K}$ isomorphic to the extension triangle of some semi-split extension as being exact. Then the category $\mathfrak{K} \mathfrak{K}$ together with the suspension automorphism and the specified class of exact triangles is a triangulated category.

\section{The bootstrap class}

Definition 3.15. The bootstrap class $\mathcal{B} \subset \mathfrak{K} \mathfrak{K}$ is the localizing subcategory in $\mathfrak{K} \mathfrak{K}$ generated by the object $\mathbb{C} \in \mathfrak{K} \mathfrak{K}$, that is, $\mathcal{B}=\langle\mathbb{C}\rangle$.

There is another equivalent characterization of the bootstrap class by Jonathan Rosenberg and Claude Schochet which underlines its importance. For this, recall that the $\mathrm{K}_{*}$-theory functor descends to the category $\mathfrak{K} \mathfrak{K}$ and is naturally isomorphic to the representable functor $\mathrm{KK}_{*}(\mathbb{C},-)$. So the K-theory functor is a coproduct-preserving, stable, homological functor from $\mathfrak{K} \mathfrak{K}$ to the abelian category $\mathfrak{A b}_{c}^{\mathbb{Z} / 2}$ of $\mathbb{Z} / 2$-graded countable abelian groups.

Then the Universal Coefficient Theorem for $\mathfrak{K} \mathfrak{K}$ reads

Theorem 3.16 (Rosenberg-Schochet [36]). Let $A$ be a separable $\mathrm{C}^{*}$-algebra. Then $A \in \mathcal{B}$ if and only if, for all $B \in \mathfrak{K} \mathfrak{K}$, there is a short exact sequence of $\mathbb{Z} / 2$-graded abelian groups

$$
\operatorname{Ext}^{1}\left(\mathrm{~K}_{*+1}(A), \mathrm{K}_{*}(B)\right) \hookrightarrow \mathfrak{K} \mathfrak{K}_{*}(A, B) \rightarrow \operatorname{Hom}\left(\mathrm{K}_{*}(A), \mathrm{K}_{*}(B)\right),
$$

where the second map is the $\mathrm{K}$-theory functor. This sequence is natural and splits unnaturally.

Here Hom and Ext denote the graded morphism and extension groups computed in $\mathfrak{A b}_{c}^{\mathbb{Z} / 2}$.

Theorem 3.16 is very useful. For example, it implies that $\mathrm{C}^{*}$-algebras in the bootstrap class are completely determined up to $\mathfrak{K} \mathfrak{K}$-equivalence by their K-theory.

Also as a corollary, a $\mathrm{C}^{*}$-algebra is in the bootstrap class if and only if it is equivalent to a commutative $\mathrm{C}^{*}$-algebra. In particular, this means that maximal and minimal tensor products coincide in $\mathfrak{K} \mathfrak{K}$ if one of the $\mathrm{C}^{*}$-algebras is in $\mathcal{B}$. In fact, the $\mathrm{K}$-theory of this tensor product can also be computed using a short exact sequence:

Theorem 3.17 (Rosenberg-Schochet, Künneth formula [36]). Let $A$ and $B$ be separable $\mathrm{C}^{*}$-algebras with $A \in \mathcal{B}$. Then there is a short exact sequence of $\mathbb{Z} / 2$-graded abelian groups

$$
\mathrm{K}_{*}(A) \hat{\otimes} \mathrm{K}_{*}(B) \hookrightarrow \mathrm{K}_{*}(A \otimes B) \rightarrow \operatorname{Tor}\left(\mathrm{K}_{*+1}(A), \mathrm{K}_{*}(B)\right),
$$

where the first map is induced by the monoidal structure $\otimes$. This sequence is natural and splits unnaturally. 
Again, Tor denotes the graded torsion group computed in $\mathfrak{A b}_{c}^{\mathbb{Z} / 2}$ and $\hat{\otimes}$ denotes the graded tensor product.

We can derive the short exact sequences of Theorems 3.16 and 3.17 by the general machinery of relative homological algebra, since K-theory turns out to be a universal ker $\mathrm{K}_{*}$-exact functor.

\section{3 $\mathrm{C}^{*}$-Algebras with an action of a topological space}

Building on previous work of Fell [15], Tomiyama [37], Dauns-Hofmann [10] and others, the notion of a $\mathrm{C}^{*}$-algebra over a locally compact Hausdorff space was introduced by Gennadi Kasparov [22] while proving the Novikov conjecture for subgroups of Lie groups.

Accepting the philosophy of noncommutative topology and noncommutative geometry, one may think of a general $\mathrm{C}^{*}$-algebra $A$ as a set of sections of some bundle over some base space. The intuition is to find a continuous image of a space $\operatorname{Prim}(A)$ over which $A$ will turn out to fiber in a nice way. This idea is formalized by the notion of a $\mathrm{C}^{*}$-algebras over a topological space.

Definitions and proofs of all facts mentioned in this section can be found in [30] or [32].

Definition 3.18. Let $A$ be a $\mathrm{C}^{*}$-algebra. Denote by $\operatorname{Prim}(A)$ the primitive ideal space of $A$ with hull-kernel topology.

Let $X$ be a possibly non-Hausdorff topological space.

Definition 3.19. An action of a topological space $X$ on a $\mathrm{C}^{*}$-algebra $A$ is a pair $(A, \psi)$, where $\psi: \operatorname{Prim}(A) \rightarrow X$ is a continuous map.

In this situation, we say that $A$ is a $\mathrm{C}^{*}$-algebra over $X$, or simply an $X-\mathrm{C}^{*}$-algebra.

Let $\mathbb{O}(X)$ denote the lattice of open subsets in $X$ ordered by inclusion, and let $\mathbb{I}(A)$ denote the lattice of closed *-ideals in $A$, also ordered by inclusion. There is a lattice isomorphism

$$
\alpha: \mathbb{O}(\operatorname{Prim}(A)) \stackrel{\cong}{\longrightarrow} \mathbb{I}(A), \quad U \mapsto \bigcap_{\mathfrak{p} \in \operatorname{Prim}(A) \backslash U} \mathfrak{p} .
$$

For an $X$-C C $^{*}$-algebra $(A, \psi)$, this gives a map

$$
\mathbb{O}(X) \rightarrow \mathbb{I}(A), \quad U \mapsto \alpha \circ \psi^{-1}(U)=: A(U),
$$

that commutes with arbitrary suprema and finite infima in the corresponding lattices.

We can also extend these notions to locally closed subsets of $X$. Recall that a subset $Y \subseteq X$ is locally closed if and only if $Y=U \backslash V$ for open subsets $U, V \in \mathbb{O}(X)$ with $V \subseteq U$. Denote the set of all non-empty locally closed subsets of $X$ by $\mathbb{L} \mathbb{C}(X)$. 
Definition 3.20. For $Y \in \mathbb{L} \mathbb{C}(X)$, let $Y=U \backslash V$ for $U, V \in \mathbb{O}(X)$ and let $A$ be a $\mathrm{C}^{*}$-algebra over $X$. Then we define

$$
A(Y):=A(U) / A(V) .
$$

This definition does not depend on the choice of $U$ and $V$ by [30, Lemma 2.15]. Now we define morphisms of $\mathrm{C}^{*}$-algebras over $X$.

Definition 3.21. Let $A$ and $B$ be $\mathrm{C}^{*}$-algebras over a topological space $X$. An $X$-equivariant ${ }^{*}$-homomorphism is a ${ }^{*}$-homomorphism $f: A \rightarrow B$ such that $f(A(U)) \subseteq B(U)$ for all $U \in \mathbb{O}(X)$.

The category of $\mathrm{C}^{*}$-algebras over a topological space $X$ together with $X$-equivariant *-homomorphisms is denoted by $\mathfrak{C}^{*} \mathfrak{a l g}(X)$. If $X$ is a single point, there is no structure to an action and we get the category of $\mathrm{C}^{*}$-algebras and *-homomorphisms.

\section{External tensor product}

For topological spaces $X$ and $Y$, a continuous map $f: X \rightarrow Y$ induces a functor

$$
f_{*}: \mathfrak{C}^{*} \mathfrak{a l g}(X) \rightarrow \mathfrak{C}^{*} \mathfrak{a l g}(Y), \quad(A, \psi) \mapsto(A, f \circ \psi) .
$$

Let $A$ and $B$ be $\mathrm{C}^{*}$-algebras over $X$ and $Y$, respectively. Let $A \otimes B$ denote their minimal tensor product. Then there is a canonical continuous map $\operatorname{Prim}(A \otimes B) \rightarrow \operatorname{Prim}(A) \times \operatorname{Prim}(B)$. Therefore, $A \otimes B$ naturally becomes a $\mathrm{C}^{*}$-algebra over $X \times Y$, and we have a bifunctor

$$
\otimes: \mathfrak{C}^{*} \mathfrak{a l g}(X) \times \mathfrak{C}^{*} \mathfrak{a l g}(Y) \rightarrow \mathfrak{C}^{*} \mathfrak{a l g}(X \times Y), \quad A \times B \mapsto A \otimes B .
$$

In particular, if $Y$ is a single point, then $X \times Y \cong X$, giving a bifunctor

$$
\otimes: \mathfrak{C}^{*} \mathfrak{a l g}(X) \times \mathfrak{C}^{*} \mathfrak{a l g} \rightarrow \mathfrak{C}^{*} \mathfrak{a l g}(X),
$$

where $\mathfrak{C}^{*} \mathfrak{a l g}$ denotes the category of $\mathrm{C}^{*}$-algebras and *-homomorphisms.

\section{Sober spaces}

Recall that an irreducible closed subset of a space $X$ is a closed subset of $X$ that is non-empty and not a union of two proper closed subsets of itself.

Definition 3.22. A sober space $X$ is a topological space $X$ such that every irreducible closed subset of $X$ is the closure of exactly one singleton of $X$.

An equivalent characterization of a sober space is that it can be recovered from its lattice of open subsets.

For every topological space $X$, there exists its sober completion $\hat{X}$. Since morphisms in $\mathfrak{C}^{*} \mathfrak{a l g}(X)$ only use $\mathbb{O}(X)$, we have an equivalence of categories $\mathfrak{C}^{*} \mathfrak{a l g}(X) \cong \mathfrak{C}^{*} \mathfrak{a l g}(\hat{X})$. So, for our purposes, we always assume the topological space $X$ to be sober. 
If $X$ is sober, there is a one-to-one correspondence between continuous maps $\operatorname{Prim}(A) \rightarrow X$ and maps $\mathbb{O}(X) \rightarrow \mathbb{I}(A)$ that commute with arbitrary suprema and finite infima. This correspondence is exactly the one given by (3.1), so we may use the latter map to define $X$-C $\mathrm{C}^{*}$-algebras.

So, for a sober space $X$, a $C^{*}$-algebra over $X$ is a pair $\left(A, \psi^{*}\right)$, where $A$ is a $\mathrm{C}^{*}$-algebra and

$$
\psi^{*}: \mathbb{O}(X) \rightarrow \mathbb{I}(A), \quad U \mapsto A(U),
$$

is a map that preserves arbitrary suprema and finite infima. In particular, for all $U, V \in \mathbb{O}(X)$ with $U \subseteq V$ this gives the monotonicity condition $A(U) \triangleleft A(V)$, and $A(\emptyset)=0$ and $A(X)=A$.

\section{The $X$-equivariant Kasparov category}

We call a $\mathrm{C}^{*}$-algebra $(A, \psi)$ over $X$ separable if $A$ is a separable $\mathrm{C}^{*}$-algebra.

Definition 3.23. Let $X$ be a topological space. Let $A$ and $B$ be separable $\mathrm{C}^{*}$-algebras over $X$. A Kasparov cycle $\left(\varphi, \mathcal{H}_{B}, F\right)$ is $X$-equivariant if

$$
\varphi(A(U)) \cdot \mathcal{H}_{B} \subseteq \mathcal{H}_{B} \cdot B(U)
$$

for every $U \in \mathbb{O}(X)$. Homotopy of $X$-equivariant Kasparov cycles is defined as in Definition 3.13.

Let $\mathrm{KK}_{*}(X ; A, B)$ denote the graded abelian group of homotopy classes of $X$-equivariant Kasparov cycles from $A$ to $B$, with addition given by direct sum.

The groups $\mathrm{KK}_{*}(X ; A, B)$, as their non-equivariant counterparts, also define a Hom-like bifunctor from the category of separable $X$-C*-algebras to $\mathbb{Z} / 2$ graded abelian groups, that is, it is covariant in the first and contravariant in the second variable and there is a natural associative Kasparov product

$$
\mathrm{KK}_{i}(X ; A, B) \times \mathrm{KK}_{j}(X ; B, C) \rightarrow \mathrm{KK}_{i+j}(X ; A, C) .
$$

Definition 3.24. Let $\mathfrak{K} \mathfrak{K}(X)$ be the category with objects separable $\mathrm{C}^{*}$ algebras over $X$ and with morphism sets $\mathrm{KK}_{0}(X ; A, B)$.

If the space $X$ is homeomorphic to a point, we recover the non-equivariant Kasparov category $\mathfrak{K} \mathfrak{K}$. The external tensor product of $\mathrm{C}^{*}$-algebras over a space descends to an external tensor product on the corresponding Kasparov categories. In particular, we have a bifunctor

$$
\mathfrak{K} \mathfrak{K}(X) \otimes \mathfrak{K} \mathfrak{K} \rightarrow \mathfrak{K} \mathfrak{K}(X) .
$$

$X$-equivariant Kasparov theory enjoys similar properties as the classical version; namely, $\mathfrak{K} \mathfrak{K}(X)$ is a stable additive category with countable $\mathrm{C}_{0}$-direct sums as coproducts. The stability is with respect to the suspension

$$
\Sigma: \mathfrak{K} \mathfrak{K}(X) \rightarrow \mathfrak{K} \mathfrak{K}(X), \quad A \mapsto \mathrm{C}_{0}(\mathbb{R}) \otimes A,
$$

which is an automorphism because of Bott periodicity. 


\section{Triangulated structure}

A triangulated structure in $\mathfrak{K} \mathfrak{K}(X)$ is constructed as for $\mathfrak{K} \mathfrak{K}$.

An extension of $\mathrm{C}^{*}$-algebras over $X$ is a diagram $I \rightarrow E \rightarrow Q$ where $I(U) \hookrightarrow E(U) \rightarrow Q(U)$ is an extension of $\mathrm{C}^{*}$-algebras for all $U \in \mathbb{O}(X)$. If an extension splits by an $X$-equivariant *-homomorphism, we call it a split extension; we call it semi-split if there is a completely positive, contractive, $X$-equivariant section $Q \rightarrow E$.

Note that an extension of $\mathrm{C}^{*}$-algebras over $X$ also gives corresponding extensions for all locally closed subsets of $X$. These extensions are also (semi) split if the original extension was.

Exactly as for $\mathfrak{K} \mathfrak{K}$, for every semi-split extension $I \hookrightarrow E \rightarrow Q$ for separable $X$ $\mathrm{C}^{*}$-algebras $I, E$ and $Q$, there exists a unique map $\Sigma Q \rightarrow I$ and an isomorphism $I \stackrel{\cong}{\rightarrow} \operatorname{cone}(E \rightarrow Q)$, both in $\mathfrak{K} \mathfrak{K}(X)$, such that the following diagram is an isomorphism of triangles:

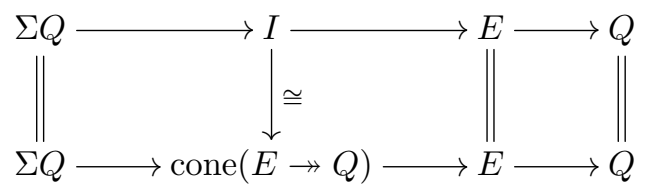

Here the mapping cone in $\mathfrak{C}^{*} \mathfrak{a l g}(X)$ is defined in the same way as the classical version, with *-homomorphisms replaced with $X$-equivariant ones.

The first triangle in the above diagram is called the extension triangle of a semi-split extension $I \hookrightarrow E \rightarrow Q$.

Declare a triangle in $\mathfrak{K} \mathfrak{K}(X)$ exact if it is isomorphic to the extension triangle of some semi-split extension. Then the category $\mathfrak{K} \mathfrak{K}(X)$, together with the specified class of exact triangles and the suspension automorphism $\Sigma$, is a triangulated category.

\section{Filtrated K-theory}

In this section, we give examples of the constructions of Section 2. All the facts and results recalled here can be found in [32].

We want to restrict attention to $\mathfrak{K} \mathfrak{K}(X)$ in case $X$ is finite. As mentioned earlier, it makes no difference for our purposes if we assume $X$, in addition, to be sober. This is not an unreasonable restriction also in general, since a finite topological space is sober if and only if it is $T_{0}$.

So, from now on, assume $X$ is finite and $T_{0}$.

Definition 3.25. For every locally closed set $Y \in \mathbb{L} \mathbb{C}(X)$, define a functor

$$
\mathrm{FK}_{Y}: \mathfrak{K} \mathfrak{K}(X) \rightarrow \mathfrak{A} \mathfrak{b}^{\mathbb{Z} / 2}, \quad \mathrm{FK}_{Y}(A):=\mathrm{K}_{*}(A(Y)) .
$$

By definition, $\mathrm{FK}_{Y}$ is a stable homological functor for every $Y \in \mathbb{L} \mathbb{C}(X)$.

Meyer-Nest combine the functors $\mathrm{FK}_{Y}$ for all $Y \in \mathbb{L} \mathbb{C}(X)$ into a single filtrated K-theory functor. The latter, however, also includes its target category, which we recall below. 
First, let $\mathcal{N} \mathcal{T}$ be the small, $\mathbb{Z} / 2$-graded, pre-additive category with object set $\mathbb{L} \mathbb{C}(X)$, and arrow space $\mathcal{N} \mathcal{T}_{*}(Y, Z)$ equal to the $\mathbb{Z} / 2$-graded abelian group of natural transformations $\mathrm{FK}_{Y} \Rightarrow \mathrm{FK}_{Z}$.

Now let $\mathfrak{M o d}(\mathcal{N} \mathcal{T})$ be the abelian category of grading-preserving, additive functors $\mathcal{N} \mathcal{T} \rightarrow \mathfrak{A b}^{\mathbb{Z} / 2}$.

We may think of $\mathcal{N} \mathcal{T}$ as a $\mathbb{Z} / 2$-graded ring, with underlying set the direct sum of $\mathcal{N} \mathcal{T}_{*}(Y, Z)$ for all $Y, Z \in \mathbb{L} \mathbb{C}(X)$. The product is defined as a composition of morphisms and as zero when the latter is not possible. Then additive functors $\mathcal{N} \mathcal{T} \rightarrow \mathfrak{A b}^{\mathbb{Z} / 2}$ become actual $\mathbb{Z} / 2$-graded modules over this graded ring. So we will refer to them as $\mathcal{N} \mathcal{T}$-modules. This also explains the notation.

Definition 3.26. The filtrated K-theory is the functor

$$
\mathrm{FK}=\left(\mathrm{FK}_{Y}\right)_{Y \in \mathbb{L} \mathbb{C}(X)}: \mathfrak{K} \mathfrak{K}(X) \rightarrow \mathfrak{M o d}(\mathcal{N} \mathcal{T})_{c}, \quad A \mapsto\left(\mathrm{K}_{*}(A(Y))\right)_{Y \in \mathbb{L} \mathbb{C}(X)},
$$

where $\mathfrak{M o d}(\mathcal{N} \mathcal{T})_{c}$ is the full subcategory of countable modules in $\mathfrak{M} \mathfrak{o d}(\mathcal{N} \mathcal{T})$.

We have a representability theorem for the functors in Definition 3.25:

Theorem 3.27 (Meyer-Nest [32, Theorem 2.5]). Let $X$ be a finite topological space. The covariant functors $\mathrm{FK}_{Y}$ for $Y \in \mathbb{L} \mathbb{C}(X)$ are representable, that is, there are objects $\mathcal{R}_{Y} \in \mathfrak{K} \mathfrak{K}(X)$ and natural isomorphisms

$$
\mathrm{KK}_{*}\left(X ; \mathcal{R}_{Y}, A\right) \cong \mathrm{FK}_{Y}(A)=\mathrm{K}_{*}(A(Y))
$$

for all $A \in \mathfrak{K} \mathfrak{K}(X), Y \in \mathbb{L} \mathbb{C}(X)$.

We also need to recall the explicit description of the objects $\mathcal{R}_{Y}$ for $Y \in$ $\mathbb{L} \mathbb{C}(X)$, since we will be dealing with them quite frequently. For this we define the specialization preorder $\preceq$ on $X$ : for any $x, y \in X$,

$$
x \preceq y \Longleftrightarrow \overline{\{x\}} \subseteq \overline{\{y\}} .
$$

Since $X$ is finite and $T_{0}$, this preorder totally determines the topology; namely, a subset $Y \subseteq X$ is open if and only if for every $y$ in $Y, y \preceq x$ implies $x \in Y$, and it is closed if and only if for every $y \in Y, x \preceq y$ implies $x \in Y$. Then a subset $Y \subseteq X$ is locally closed if and only if $x \preceq y \preceq z$ and $x, z \in Y$ implies $y \in Y$. The topology given by a preorder in this fashion is called Alexandrov topology.

Let $\operatorname{Ch}(X)$ be a simiplicial set with the chains $x_{0} \preceq x_{1} \preceq \cdots \preceq x_{n}$ as $n$-simplices, with face maps deleting an entry of the chain and with degeneracy maps doubling it. Denote by $S_{X}$ the set of all strict chains in $X$. For every strict chain $I=\left(x_{0} \prec x_{1} \prec \cdots \prec x_{n}\right)$, let $\Delta_{I}$ be a copy of $\Delta_{n}$, the standard $n$-simplex. Also, let $\Delta_{I}^{\mathrm{o}}:=\Delta_{n} \backslash \partial \Delta_{n}$ be the open simplex. Then the underlying set of the geometric realization of $\operatorname{Ch}(X)$ (for which we also write $\operatorname{Ch}(X)$ ) can be represented as a disjoint union

$$
\operatorname{Ch}(X)=\coprod_{I \in S_{X}} \Delta_{I}^{\mathrm{o}}
$$


Let $X^{\text {op }}$ be $X$ with the Alexandrov topology of the reversed partial order $\succeq$. Then, because of the disjoint union decomposition above, one can construct a continuous map [32, Proposition 2.8]

$$
(m, M): \operatorname{Ch}(X) \rightarrow X^{\mathrm{op}} \times X,
$$

where for $x \in \Delta_{I}^{\mathrm{o}}$, we define $m(x)=\min I$ and $M(x)=\max I$, where $\min I$ and $\max I$ are the minimal and the maximal elements of the chain $I$ in $S_{X}$, respectively. Since

Let $\mathcal{R}:=\mathrm{C}(\mathrm{Ch}(X))$, the $\mathrm{C}^{*}$-algebra of continuous functions on $\mathrm{Ch}(X)$.

$$
\operatorname{Prim} \mathcal{R}=\operatorname{Prim} \mathrm{C}(\mathrm{Ch}(X)) \cong \mathrm{Ch}(X),
$$

the map $(m, M)$ turns $\mathcal{R}$ into a $\mathrm{C}^{*}$-algebra over $X^{\mathrm{op}} \times X$.

Definition 3.28. Let $\mathcal{R}_{Y}$ be the restriction of $\mathcal{R}$ to $Y^{\mathrm{op}} \times X$, viewed as an $X$-C ${ }^{*}$-algebra via the coordinate projection $Y^{\mathrm{op}} \times X \rightarrow X$, where $Y^{\mathrm{op}} \subseteq X^{\mathrm{op}}$.

In other words,

$$
\mathcal{R}_{Y}(Z):=\mathcal{R}\left(Y^{\mathrm{op}} \times Z\right)=\mathrm{C}_{0}\left(m^{-1}(Y) \cap M^{-1}(Z)\right) .
$$

The objects $\mathcal{R}_{Y}$ for $Y \in \mathbb{L} \mathbb{C}(X)$ generate an important class of triangles in $\mathfrak{K} \mathfrak{K}(X)$. Let $Y \in \mathbb{L} \mathbb{C}(X)$ and $U \in \mathbb{O}(Y)$. Then $m^{-1}(Y \backslash U)$ is open in $m^{-1}(Y)$, and for any $Z \in \mathbb{L} \mathbb{C}(X)$ we have a $\mathrm{C}^{*}$-algebra extension

$$
\begin{aligned}
\mathrm{C}_{0}\left(m^{-1}(Y \backslash U) \cap M^{-1}(Z)\right) \hookrightarrow \mathrm{C}_{0}\left(m^{-1}(Y)\right. & \left.\cap M^{-1}(Z)\right) \\
& \rightarrow \mathrm{C}_{0}\left(m^{-1}(U) \cap M^{-1}(Z)\right) .
\end{aligned}
$$

Moreover, there exists a completely positive and contractive section $\mathrm{C}_{0}\left(m^{-1}(U) \cap\right.$ $\left.M^{-1}(Z)\right) \rightarrow \mathrm{C}_{0}\left(m^{-1}(Y) \cap M^{-1}(Z)\right)$. Therefore, by definition

$$
\mathcal{R}_{Y \backslash U} \hookrightarrow \mathcal{R}_{Y} \rightarrow \mathcal{R}_{U}
$$

is a semi-split extension of $X$-C $\mathrm{C}^{*}$-algebras.

Lemma 3.29. Let $Y \in \mathbb{L} \mathbb{C}(X)$ and $U \in \mathbb{O}(Y)$. Then the triangle

$$
\Sigma \mathcal{R}_{U} \rightarrow \mathcal{R}_{Y \backslash U} \rightarrow \mathcal{R}_{Y} \rightarrow \mathcal{R}_{U}
$$

is exact in $\mathfrak{K} \mathfrak{K}(X)$.

Now we want to view this construction through the lens of relative homological algebra in $\mathfrak{K} \mathfrak{K}(X)$. The starting point is the homological ideal defined by the filtrated K-theory functor

$$
\mathfrak{I}:=\bigcap_{Y \in \mathbb{L} \mathbb{C}(X)} \operatorname{ker} \mathrm{FK}_{Y} .
$$

Once we fix $\mathfrak{I}$, the following theorem naturally leads us to consider the ring of natural transformations $\mathcal{N} \mathcal{T}$ and the filtrated K-theory functor. 
3. Relevant examples

Theorem 3.30 (Meyer-Nest [32, Theorem 4.4]). The filtrated K-theory FK : $\mathfrak{K} \mathfrak{K}(X) \rightarrow \mathfrak{M o d}(\mathcal{N} \mathcal{T})_{c}$ is the universal $\mathfrak{I}$-exact stable homological functor.

The next step is to define an analogue of a bootstrap subcategory in $\mathfrak{K} \mathfrak{K}(X)$. As it turns out, the localizing subcategory generated by I-projective objects, that is, $\left\langle\mathfrak{P}_{\mathfrak{J}}\right\rangle$, is a good candidate. However, first we would like to define it independently of $\mathfrak{I}$.

For every point in $X$, consider the inclusion $\{x\} \hookrightarrow X$. This defines a $\mathrm{C}^{*}$-algebra $i_{x}(\mathbb{C}):=(\mathbb{C}, x)$ over $X$, where by $x$ we denote the map $\operatorname{Prim}(\mathbb{C}) \cong$ $\{x\} \hookrightarrow X$. In other words,

$$
i_{x}(\mathbb{C})(Y)= \begin{cases}\mathbb{C} & \text { if } x \in Y, \\ 0 & \text { otherwise }\end{cases}
$$

for all $Y \in \mathbb{L} \mathbb{C}(X)$.

Definition 3.31. The bootstrap category $\mathcal{B}(X) \subset \mathfrak{K} \mathfrak{K}(X)$ is the localizing subcategory of $\mathfrak{K} \mathfrak{K}(X)$ generated by the objects $i_{x}(\mathbb{C})$ for $x \in X$.

Proposition 3.32 (Meyer-Nest [32, Proposition 4.3]). The bootstrap category is the localizing subcategory of $\mathfrak{K} \mathfrak{K}(X)$ that is generated by $\mathfrak{I}$-projective objects, that is, $\mathcal{B}(X) \cong\left\langle\mathfrak{P}_{\mathfrak{I}}\right\rangle$.

We could go on now and produce a Universal Coefficient Theorem using the general machinery of Theorem 2.12 for finite $X$. However, since we will mainly deal with a more concrete case, we now restrict our attention to a more specialized class of spaces.

\section{Filtrations}

Say the preordered set corresponding to a finite space $X$ is a totally ordered set of $n$ elements. For a $T_{0}$ space (this is always assumed), this is equivalent to $X$ having a totally ordered lattice of open subsets. In other words, $X=\{1, \ldots, n\}$ and every open set is of the form

$$
[a, n]:=\{x \in X \mid a \leq x \leq n\} \quad \text { for some } a \in X .
$$

From now on, assume $X$ to be such a space.

By (3.2), a $\mathrm{C}^{*}$-algebra over $X$ is a $\mathrm{C}^{*}$-algebra $A$ together with ideals $I_{a}:=A([a, n])$ for every open set in $[a, n] \in \mathbb{O}(X)$. By the monotonicity condition, this amounts to a filtration

$$
I_{n} \triangleleft I_{n-1} \triangleleft \cdots \triangleleft I_{2} \triangleleft I_{1} \cong A .
$$

An $X$-equivariant *-homomorphism $f: A \rightarrow A^{\prime}$ of two $\mathrm{C}^{*}$-algebras over $X$ by definition maps $f\left(I_{a}\right) \subseteq I_{a}^{\prime}$ for every $a \in X$. Therefore, we exactly get the maps of filtrations. We conclude that the category of $\mathrm{C}^{*}$-algebras over a finite space $X$ with totally ordered lattice of open subsets is equivalent to the 
category of filtrations of $\mathrm{C}^{*}$-algebras of fixed length. The latter category is of main interest to us.

Any locally closed subset $Y=U \backslash V$, for $U, V \in \mathbb{O}(X)$, is of the form

$$
Y=[a, b]:=\{y \in X \mid a \leq y \leq b\}
$$

for some $a, b \in X$ with $a \leq b$. Then $A(Y)=A([a, b])=I_{a} / I_{b+1}$.

Now we would like to explicitly compute the category $\mathcal{N} \mathcal{T}$ for $\mathfrak{K} \mathfrak{K}(X)$. For this we need to find all natural transformations of functors $\mathrm{FK}_{Y} \Rightarrow \mathrm{FK}_{Z}$ for $Y, Z \in \mathbb{L} \mathbb{C}(X)$. We can actually guess some of them. For $Y \in \mathbb{L} \mathbb{C}(X)$, if $U \in \mathbb{O}(Y)$, the $\mathrm{C}^{*}$-algebra extension $A(U) \hookrightarrow A(Y) \rightarrow A(Y \backslash U)$ gives a natural six-term exact sequence

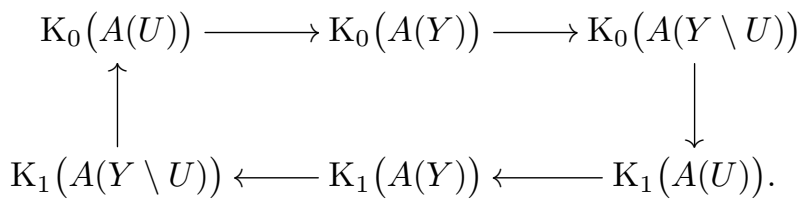

By definition, this leads to natural transformations $\mathrm{FK}_{U} \Rightarrow \mathrm{FK}_{Y} \Rightarrow \mathrm{FK}_{Y \backslash U} \Rightarrow$ $\mathrm{FK}_{U}[1]$, where [1] denotes the degree exchanging functor on $\mathfrak{A b}^{\mathbb{Z} / 2}$. It turns out that these generate all natural transformations in our case.

Theorem 3.27 together with the Yoneda Lemma gives

$$
\begin{aligned}
\mathcal{N} \mathcal{T}_{*}(Y, Z) & \cong \mathrm{KK}_{*}\left(X ; \mathcal{R}_{Z}, \mathcal{R}_{Y}\right) \cong \mathrm{FK}_{Z}\left(\mathcal{R}_{Y}\right)=\mathrm{K}_{*}\left(\mathcal{R}_{Y}(Z)\right) \\
& =\mathrm{K}_{*}\left(\mathcal{R}\left(Y^{\mathrm{op}} \times Z\right)\right)=\mathrm{K}^{*}\left(m^{-1}(Y) \cap M^{-1}(Z)\right) .
\end{aligned}
$$

In our case, $\operatorname{Ch}(X)$ is an $(n-1)$-dimensional closed simplex. Let $\Delta_{[a, b]}$ denote the $(b-a)$-dimensional face of $\operatorname{Ch}(X)$ corresponding to $[a, b] \in \mathbb{L} \mathbb{C}(X)$. Then for the map

$$
(m, M): \mathrm{Ch}(X) \rightarrow X^{\mathrm{op}} \times X,
$$

we have $m(x)=a$ and $M(x)=b$ for $x \in \Delta_{[a, b]}^{o}$.

Therefore, computing $\mathcal{N} \mathcal{T}_{*}(Y, Z)$ comes down to computing the topological $\mathrm{K}$-theory of some simplicial complexes. For $Y=[a, b]$ and $Z=[c, d]$, one gets [32, Section 3.1]:

$$
\mathcal{N} \mathcal{T}_{*}(Y, Z) \cong \mathrm{K}_{*}\left(\mathcal{R}_{[a, b]}([c, d])\right) \cong \begin{cases}\mathbb{Z}[0] & \text { if } c \leq a \leq d \leq b, \\ \mathbb{Z}[1] & \text { if } a<c \text { and } b<d \text { and } c-1 \leq b, \\ 0 & \text { otherwise }\end{cases}
$$

where $\mathbb{Z}[0]$ denotes the $\mathbb{Z} / 2$-graded abelian group $(\mathbb{Z}, 0) \in \mathfrak{A b}^{\mathbb{Z} / 2}$ and $\mathbb{Z}[1]$ is the shorthand for $(\mathbb{Z}[0])[1]$. These are exactly the expected conditions from (3.3).

The conditions (3.4) give a handy way to diagrammatically depict the category $\mathcal{N} \mathcal{T}$ (see Figure 2.1). In the diagram, every arrow $Y \rightarrow Z$ for $Y, Z \in \mathbb{L} \mathbb{C}(X)$ represents a generator of the category $\mathcal{N} \mathcal{T}_{*}(Y, Z)$; general elements in $\mathcal{N} \mathcal{T}_{*}(Y, Z)$ are paths in Figure 2.1. The squares commute and the 


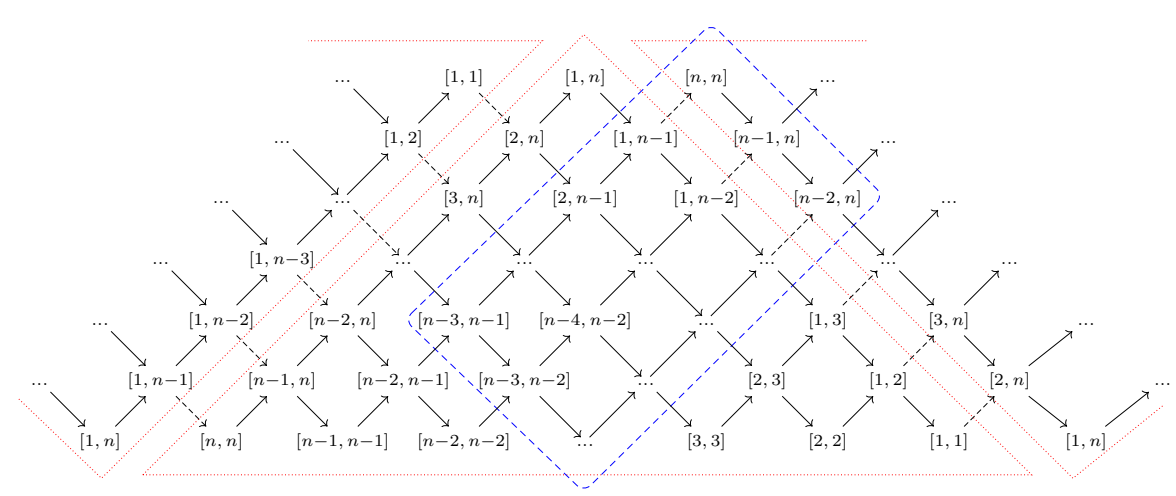

Figure 2.1: The invariant triangle is marked with dotted lines. Dashed arrows are odd. The dashed square represents the set $B_{Y}$ for $Y=[n-3, n-1]$.

diagram is assumed to be filled with zeros outside the drawn "strip". A product of generators is 0 if and only if it factors through one of the zeros outside the strip, and this gives all relations among the generators. Dashed arrows represent degree one maps. Figure 2.1 really shows how $\mathcal{N} \mathcal{T}$ is generated by the maps from the six-term exact sequences (3.3), and how it is represented by what we will call an invariant triangle diagram. This triangle diagram, marked with dotted lines, maps to the flipped version of itself infinitely via degree shifting maps.

By the classical Universal Coefficient Theorem, the K-theory functor is a complete invariant for the classical bootstrap class $\mathcal{B} \cong\langle\mathbb{C}\rangle$ of $\mathrm{C}^{*}$-algebras. So since $\mathrm{K}_{*}(\mathbb{C}) \cong \mathbb{Z}[0]$ and $\mathrm{K}_{*}(\Sigma \mathbb{C})=\mathrm{K}_{*}\left(\mathrm{C}_{0}(\mathbb{R})\right) \cong \mathbb{Z}[1]$, equation (3.4) gives

$$
\mathcal{R}_{[a, b]}([c, d]) \cong \begin{cases}\mathbb{C} & \text { if } c \leq a \leq d \leq b, \\ \mathrm{C}_{0}(\mathbb{R}) & \text { if } a<c \text { and } b<d \text { and } c-1 \leq b, \\ 0 & \text { otherwise. }\end{cases}
$$

In the bootstrap class, the objects $\mathcal{R}_{Y}$ for $Y \in \mathbb{L} \mathbb{C}(X)$ are completely determined by the conditions (3.4). Since $\mathrm{K}_{*}\left(\mathcal{R}_{Y}(Z)\right) \cong \mathcal{N} \mathcal{T}_{*}(Y, Z)$, the $X$ $\mathrm{C}^{*}$-algebra $\mathcal{R}_{Y}$ is represented in this diagram by a "maximal box" starting at $Y$ : a subdiagram of all $Z \in \mathbb{L} \mathbb{C}(X)$ to which the group of morphisms from $Y$ in $\mathcal{N} \mathcal{T}$ is non-zero. We denote the set of all such $Z$ by $B_{Y}$ (see Figure 2.1); so

$$
B_{Y}:=\left\{Z \in \mathbb{L} \mathbb{C}(X) \mid \mathrm{K}_{*}\left(\mathcal{R}_{Y}(Z)\right) \varsubsetneqq 0\right\} .
$$

We will also frequently use the localized version of these representative elements. Let $p \in \operatorname{Spec} \mathbb{Z}$; recall that $\mathbb{F}_{p}=\mathbb{Z} / p$ for $p \neq 0$, and $\mathbb{F}_{p}=\mathbb{Q}$ for $p=0$.

When we say that an object in the Kasparov category or the bootstrap class is unique, we of course mean the uniqueness up to KK-equivalence.

Definition 3.33. For $p \in \operatorname{Spec} \mathbb{Z}$, let $\kappa(p)$ be the unique $\mathrm{C}^{*}$-algebra in $\mathcal{B}$ with $\mathrm{K}_{*}(\kappa(p)) \cong \mathbb{F}_{p}[0]$. 
Definition 3.34. For $p \in \operatorname{Spec} \mathbb{Z}$ and $Y \in \mathbb{L} \mathbb{C}(X)$, let

$$
\mathcal{R}_{Y}^{p}:=\mathcal{R}_{Y} \otimes \kappa(p) .
$$

Remark 3.35. Since $\mathrm{K}_{*}\left(\mathcal{R}_{Y}\right)$ is torsion-free, the Künneth formula gives

$$
\mathrm{K}_{*}\left(\mathcal{R}_{Y}^{p}\right)=\mathrm{K}_{*}\left(\mathcal{R}_{Y} \otimes \kappa(p)\right) \cong \mathrm{K}_{*}\left(\mathcal{R}_{Y}\right) \otimes_{\mathbb{Z}} \mathrm{K}_{*}(\kappa(p)) .
$$

So we get the same conditions as (3.4) and (3.5) for $\mathcal{R}_{[a, b]}^{p}([c, d])$, but with $\mathbb{Z}[i]$ replaced by $\mathbb{F}_{p}[i]$ and $\mathbb{C}[i]$ by $\kappa(p)[i]$ for $i=0,1$.

For classification purposes, it is important to characterize $\mathcal{N} \mathcal{T}$-modules in the image of the filtrated K-theory functor. We see that these modules should have certain exactness properties coming from (3.3). Following this, we define

Definition 3.36. An $\mathcal{N} \mathcal{T}$-module $M$ is exact if the chain complexes

$$
\cdots \longrightarrow M(U) \longrightarrow M(Y) \longrightarrow M(Y \backslash U) \longrightarrow M(U) \longrightarrow \cdots
$$

are exact for all $Y \in \mathbb{L} \mathbb{C}(X), U \in \mathbb{O}(Y)$ with maps coming from the generators in (3.4).

Meyer-Nest show that exact modules also behave nice homologically:

Theorem 3.37 (Meyer-Nest [32, Theorem 4.9]). Let $M \in \mathfrak{M o d}(\mathcal{N} \mathcal{T})_{\mathrm{c}}$. Then $M=\mathrm{FK}(A)$ for some $A \in \mathfrak{K} \mathfrak{K}(X)$ if and only if $M$ is exact and if and only if $M$ has a projective resolution of length 1 as an $\mathcal{N} \mathcal{T}$-module.

As for the Universal Coefficient Theorem, putting together Theorem 3.37 and Theorem 2.12 gives

Theorem 3.38 (Meyer-Nest [32, Theorem 4.10]). For any $A \in \mathcal{B}(X)$ and $B \in \mathfrak{K} \mathfrak{K}(X)$, there are natural short exact sequences

$\operatorname{Ext}_{\mathcal{N} \mathcal{T}}^{1}(\operatorname{FK}(A)[j+1], \operatorname{FK}(B)) \hookrightarrow \operatorname{KK}_{j}(X ; A, B) \rightarrow \operatorname{Hom}_{\mathcal{N} \mathcal{T}}(\operatorname{FK}(A)[j], \operatorname{FK}(B))$

for $j \in \mathbb{Z} / 2$, where $[j]$ and $[j+1]$ denote degree shifts.

In the classical case, as a corollary to the Universal Coefficient Theorem, $\mathrm{K}$-theory is a complete invariant for $\mathrm{C}^{*}$-algebras in $\mathcal{B}$. This also uses the property that the category of abelian groups is hereditary, that is, all abelian groups have one-step projective resolutions. This corollary is one of the main appeals of a Universal Coefficient Theorem, since it allows to classify certain $\mathrm{C}^{*}$-algebras by their K-theory. As a parallel in our setting, we get

Corollary 3.39 (Meyer-Nest $\left[32\right.$, Theorem 4.8]). Let $M \in \mathfrak{M o d}(\mathcal{N} \mathcal{T})_{c}$ have a projective resolution of length 1 . Then there is $A \in \mathcal{B}(X)$ with $\operatorname{FK}(A) \cong M$, and this object is unique up to isomorphism in $\mathcal{B}(X)$. 
3. Relevant examples

\section{Direct sum decomposition}

We are also interested in which exact modules are the K-theories of representable objects $\mathcal{R}_{Y}$ for $Y \in \mathbb{L} \mathbb{C}(X)$.

Definition 3.40. The free $\mathcal{N} \mathcal{T}$-module on $Y$, for $Y \in \mathbb{L} \mathbb{C}(X)$, is defined by

$$
Q_{Y}(Z):=\mathcal{N} \mathcal{T}_{*}(Y, Z) \quad \text { for every } Z \in \mathbb{L} \mathbb{C}(X) .
$$

An $\mathcal{N} \mathcal{T}$-module is free if it is isomorphic to a direct sum of degree-shifted free modules $Q_{Y}[j], j \in \mathbb{Z} / 2$.

Theorem 3.41 (Meyer-Nest [32, Theorem 3.12]). Let $M \in \mathfrak{M o d}(\mathcal{N} \mathcal{T})_{\mathrm{c}}$. Then $M$ is a free $\mathcal{N} \mathcal{T}$-module if and only if $M(Y)$ is a free abelian group for all $Y \in \mathbb{L} \mathbb{C}(X)$ and $M$ is exact.

This theorem is a consequence of the fact that in case $M(Y)$ is free for all $Y \in \mathbb{L} \mathbb{C}(X)$, a 1-step projective resolution of $M$ degenerates to a length zero resolution, making $M$ itself projective and as a consequence free.

For our classification, we will use the localized version of Theorem 3.41. For $p \in \operatorname{Spec} \mathbb{Z}$, let

$$
\mathcal{N} \mathcal{T}^{p}:=\mathcal{N} \mathcal{T} \otimes_{\mathbb{Z}} \mathbb{F}_{p}[0]
$$

Definition 3.42. The free $\mathcal{N} \mathcal{T}^{p}$-module on $Y$, for $Y \in \mathbb{L} \mathbb{C}(X)$ and $p \in \operatorname{Spec} \mathbb{Z}$, is defined by

$$
Q_{Y}^{p}(Z):=\mathcal{N} \mathcal{T}_{*}^{p}(Y, Z)=\mathcal{N} \mathcal{T}_{*}(Y, Z) \otimes_{\mathbb{Z}} \mathbb{F}_{p}[0] \quad \text { for every } Z \in \mathbb{L} \mathbb{C}(X) .
$$

An $\mathcal{N} \mathcal{T}^{p}$-module is free if it is isomorphic to a direct sum of degree-shifted free modules $Q_{Y}^{p}[j], j \in \mathbb{Z} / 2$.

Even though the following theorem is not proved in the article by MeyerNest, we still give it here without a proof; the reason is that the proof is word by word the same as for Theorem 3.41, one just needs to replace the ring $\mathcal{N} \mathcal{T}$ with $\mathcal{N T}^{p}$.

Theorem 3.43. Let $M \in \mathfrak{M o d}(\mathcal{N} \mathcal{T})_{\mathrm{c}}$ and $p \in \operatorname{Spec} \mathbb{Z}$. Then $M$ is a free $\mathcal{N} \mathcal{T}^{p}$-module if and only if $M(Y)$ is an $\mathbb{F}_{p}$-vector space for all $Y \in \mathbb{L} \mathbb{C}(X)$ and $M$ is exact.

Recall that a multiset is a collection of objects in which the elements are allowed to repeat.

As an easy corollary of Theorem 3.43 we get:

Corollary 3.44. Let $M \in \mathfrak{M o d}(\mathcal{N} \mathcal{T})_{\mathrm{c}}, p \in \operatorname{Spec} \mathbb{Z}$ and $j=0,1$. Then $M \cong \bigoplus_{Y \in J} \operatorname{FK}\left(\mathcal{R}_{Y}^{p}\right)\left[j_{Y}\right]$ for some (possibly countably infinite) multiset $J$ with elements from $\mathbb{L} \mathbb{C}(X)$ if and only if $M(Y)$ is an $\mathbb{F}_{p}$-vector space for all $Y \in \mathbb{L} \mathbb{C}(X)$ and $M \cong \mathrm{FK}(A)$ for some $A \in \mathfrak{K} \mathfrak{K}(X)$. 
Proof. Say $M(Y)$ is an $\mathbb{F}_{p}$-vector space for all $Y \in \mathbb{L} \mathbb{C}(X)$ and $M \cong \operatorname{FK}(A)$ for some $A \in \mathfrak{K} \mathfrak{K}(X)$. By Theorem 3.37 the latter conditions mean that $M$ is exact. Then by Theorem 3.43, conditions (3.4) and Remark 3.35

$$
\begin{aligned}
M(Z) & \cong \bigoplus_{Y \in J} \mathcal{N} \mathcal{T}_{*}^{p}(Y, Z)\left[j_{Y}\right] \cong \bigoplus_{Y \in J} \mathcal{N} \mathcal{T}_{*}(Y, Z)\left[j_{Y}\right] \otimes_{\mathbb{Z}} \mathbb{F}_{p}[0] \\
& \cong \bigoplus_{Y \in J} \mathrm{~K}_{*}\left(\mathcal{R}_{Y}(Z)\right)\left[j_{Y}\right] \otimes_{\mathbb{Z}} \mathbb{F}_{p}[0] \cong \bigoplus_{Y \in J} \mathrm{~K}_{*}\left(\mathcal{R}_{Y}^{p}(Z)\right)\left[j_{Y}\right]
\end{aligned}
$$

Since $M=\bigoplus_{Z \in \mathbb{L C}(X)} M(Z)$, the definition of filtrated K-theory gives

$$
\begin{aligned}
M & \cong \bigoplus_{Z \in \mathbb{L} C(X)} \bigoplus_{Y \in J} \mathrm{~K}_{*}\left(\mathcal{R}_{Y}^{p}(Z)\right)\left[j_{Y}\right] \cong \bigoplus_{Z \in \mathbb{L} \mathbb{C}(X)} \bigoplus_{Y \in J} \mathrm{FK}_{Z}\left(\mathcal{R}_{Y}^{p}\right)\left[j_{Y}\right] \\
& \cong \bigoplus_{Y \in J} \bigoplus_{Z \in \mathbb{L} \mathbb{C}(X)} \mathrm{FK}_{Z}\left(\mathcal{R}_{Y}^{p}\right)\left[j_{Y}\right] \cong \bigoplus_{Y \in J} \operatorname{FK}\left(\mathcal{R}_{Y}^{p}\right)\left[j_{Y}\right]
\end{aligned}
$$

The reverse implication follows directly from Remark 3.35. 


\section{Classification}

\section{Properties of $\mathcal{B}(X)$ and cohomological support}

In this section, we collect some facts that will be used later to prove the classification theorem.

\subsection{Some general results for $\mathcal{B}(X)$}

In this subsection, $X$ denotes an arbitrary topological space.

Definition 4.1. For an abelian group $G$, let $\kappa(G)$ be the unique object in $\mathcal{B}:=\mathcal{B}(\{*\})$ with $\mathrm{K}_{0}(\kappa(G))=G$ and $\mathrm{K}_{1}(\kappa(G))=0$.

For example, in this notation $\kappa(p)=\kappa\left(\mathbb{F}_{p}\right)$.

Lemma 4.2. $\kappa(-)$ has the following properties:

(i) $\kappa\left(\bigoplus_{i \in I} G_{i}\right) \cong \bigoplus_{i \in I} \kappa\left(G_{i}\right)$;

(ii) Let $\left(G_{i}, f_{j}^{i}\right)$ be a countable inductive system and $\left(\kappa\left(G_{i}\right), \alpha_{j}^{i}\right)$ its lift by K-theory. Then $\kappa\left(\lim _{i} G_{i} \cong \operatorname{ho-} \longrightarrow \lim _{\longrightarrow} \kappa\left(G_{i}\right)\right.$.

Proof. (i) follows from additivity of K-theory.

(ii) By definition, the homotopy limit fits in an exact triangle

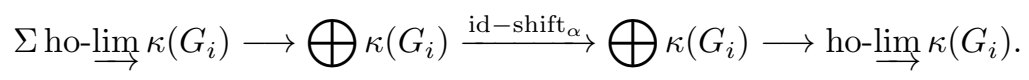

After applying the K-theory functor and decomposing the resulting exact sequence into short exact sequences, we get

$$
\operatorname{coker}\left(\mathrm{id}-\operatorname{shift}_{f}\right) \hookrightarrow \mathrm{K}_{*}\left(\text { ho-lim }_{\longrightarrow} \kappa\left(G_{i}\right)\right) \rightarrow \operatorname{ker}\left(\mathrm{id}-\operatorname{shift}_{f}\right) .
$$

Now $\operatorname{ker}\left(\mathrm{id}-\operatorname{shift}_{f}[1]\right) \cong 0$ and $\operatorname{coker}\left(\mathrm{id}-\operatorname{shift}_{f}\right) \cong \lim _{\longrightarrow} G_{i}$ by definition.

Lemma 4.3. Let $\mathcal{S} \subset \mathcal{B}(X)$ be a localizing subcategory. For any $A \in \mathcal{S}$ and $G$ a countable abelian group, we have $A \otimes \kappa(G) \in \mathcal{S}$.

Proof. First let $G$ be finitely generated. Then $G \cong \mathbb{Z}^{n} \oplus \mathbb{Z} / p_{1}^{i_{1}} \cdots \oplus \mathbb{Z} / p_{m}^{i_{m}}$. Now $A \otimes \kappa(\mathbb{Z}) \cong A \otimes \mathbb{C} \cong A \in \mathcal{S}$. Next consider the short exact sequence of $\mathbb{Z} / 2$-graded abelian groups

$$
0 \rightarrow \mathbb{Z}[0] \stackrel{p_{k}^{i_{k}}}{\longrightarrow} \mathbb{Z}[0] \rightarrow \mathbb{Z} / p_{k}^{i_{k}}[0] \rightarrow 0 .
$$

Let $\mathbb{K}$ be the algebra of compact operators on a separable infinite-dimensional Hilbert space. Since $K_{*}(\mathbb{K}) \cong \mathbb{Z}[0]$, let $\mathbb{K} \stackrel{\tilde{p}_{k}^{i_{k}}}{\longrightarrow} \mathbb{K}$ denote the map that induces 
the multiplication with $p_{k}^{i_{k}}$ in K-theory. Then (4.1) lifts to the following unique triangle in $\mathcal{B}$ :

$$
\Sigma \kappa\left(\mathbb{Z} / p_{k}^{i_{k}}\right) \longrightarrow \mathbb{K} \stackrel{\tilde{p}_{k}^{i_{k}}}{\longrightarrow} \mathbb{K} \longrightarrow \kappa\left(\mathbb{Z} / p_{k}^{i_{k}}\right) .
$$

Now $\mathbb{K}$ is KK-equivalent to the $\mathrm{C}^{*}$-algebra of complex numbers; hence the triangle (4.2) is isomorphic to the triangle

$$
\Sigma \kappa\left(\mathbb{Z} / p_{k}^{i_{k}}\right) \longrightarrow \mathbb{C} \longrightarrow \mathbb{C} \longrightarrow \kappa\left(\mathbb{Z} / p_{k}^{i_{k}}\right) .
$$

Tensoring (4.3) with $A$ leads to the triangle

$$
\Sigma\left(A \otimes \kappa\left(\mathbb{Z} / p_{k}^{i_{k}}\right)\right) \longrightarrow A \longrightarrow A \longrightarrow A \otimes \kappa\left(\mathbb{Z} / p_{k}^{i_{k}}\right) .
$$

We conclude that $A \otimes \kappa\left(\mathbb{Z} / p_{k}^{i_{k}}\right) \in \mathcal{S}$. Thus $A \otimes \kappa(G) \in \mathcal{S}$. Now let $G$ be an arbitrary countable abelian group. Then $G \cong \lim _{j \in \mathbb{N}} H_{j}$, where $H_{j} \subseteq G$ are finitely generated subgroups. We have

$$
A \otimes \kappa(G) \cong A \otimes \kappa\left(\lim _{\longrightarrow} H_{j}\right) \cong \operatorname{ho}-\lim _{\longrightarrow} A \otimes \kappa\left(H_{j}\right) \in \mathcal{S} .
$$

Corollary 4.4. For any $D \in \mathcal{B}$ and $A \in \mathcal{B}(X)$, if $A \in \mathcal{S}$ then $A \otimes D \in \mathcal{S}$.

Proof. $D \cong \kappa(G) \oplus \kappa(H)[1]$ for some abelian groups $G$ and $H$, namely, $G=$ $\mathrm{K}_{0}(D)$ and $H=\mathrm{K}_{1}(D)$.

Lemma 4.5. For all $A \in \mathcal{B}(X),\langle A\rangle \cong\langle A \otimes \kappa(p) \mid p \in \operatorname{Spec} \mathbb{Z}\rangle$.

Proof. By Lemma $4.3, A \otimes \kappa(\mathbb{Q}), A \otimes \kappa(\mathbb{Q} / \mathbb{Z}) \in\langle A\rangle$. Moreover, there is an exact triangle

$$
\Sigma(A \otimes \kappa(\mathbb{Q} / \mathbb{Z})) \longrightarrow A \longrightarrow A \otimes \kappa(\mathbb{Q}) \longrightarrow A \otimes \kappa(\mathbb{Q} / \mathbb{Z}) .
$$

So $\langle A\rangle \cong\langle A \otimes \kappa(\mathbb{Q}), A \otimes \kappa(\mathbb{Q} / \mathbb{Z})\rangle$. We also have the isomorphisms

$$
\begin{aligned}
A \otimes \kappa(\mathbb{Q} / \mathbb{Z}) \cong A \otimes \bigoplus_{p \text { prime }} \kappa\left(\mathbb{Z}\left[\frac{1}{p}\right] / \mathbb{Z}\right) & \cong \bigoplus_{p \text { prime }} A \otimes \kappa\left(\underset{n}{\lim } \mathbb{Z} / p^{n} \mathbb{Z}\right) \\
& \cong \bigoplus_{p \text { prime }} \operatorname{ho-lim} A \otimes \kappa\left(\mathbb{Z} / p^{n} \mathbb{Z}\right) .
\end{aligned}
$$

These isomorphisms, together with the exact triangles

$\Sigma\left(A \otimes \kappa\left(\mathbb{Z}\left[\frac{1}{p}\right] / \mathbb{Z}\right)\right) \rightarrow A \otimes \kappa\left(\mathbb{Z} / p^{n} \mathbb{Z}\right) \rightarrow A \otimes \kappa\left(\mathbb{Z}\left[\frac{1}{p}\right] / \mathbb{Z}\right) \stackrel{\operatorname{id} \otimes \tilde{p}^{n}}{\longrightarrow} A \otimes \kappa\left(\mathbb{Z}\left[\frac{1}{p}\right] / \mathbb{Z}\right)$

and

$\Sigma\left(A \otimes \kappa\left(\mathbb{Z} / p^{m} \mathbb{Z}\right)\right) \rightarrow A \otimes \kappa\left(\mathbb{Z} / p^{n} \mathbb{Z}\right) \stackrel{\operatorname{id} \otimes \tilde{p}^{m}}{\longrightarrow} A \otimes \kappa\left(\mathbb{Z} / p^{n+m} \mathbb{Z}\right) \rightarrow A \otimes \kappa\left(\mathbb{Z} / p^{m} \mathbb{Z}\right)$

imply that

$$
\langle A \otimes \kappa(\mathbb{Q} / \mathbb{Z})\rangle \cong\langle A \otimes \kappa(p) \mid p \in \operatorname{Spec} \mathbb{Z} \backslash\{0\}\rangle .
$$


4. Properties of $\mathcal{B}(X)$ and cohomological support

\subsection{Cohomological support}

Recall that every abelian group has a one-step minimal injective resolution, which is unique up to isomorphism. Also, every injective abelian group is isomorphic to a direct sum of indecomposable ones, namely, $\mathbb{Q}$ and $\mathbb{Z}\left[\frac{1}{p}\right] / \mathbb{Z}$, where $p$ is a prime number [26]. All this naturally extends to graded abelian groups.

Let $p \in \operatorname{Spec} \mathbb{Z}$. We say that $p$ appears in a minimal injective resolution of the abelian group $G$ if $\mathbb{Z}\left[\frac{1}{p}\right] / \mathbb{Z}$ for $p \neq 0$, and $\mathbb{Q}$ for $p=0$, appears in degree zero or one in the direct sum decomposition of the minimal injective resolution of $G$. We define

$\operatorname{supp}_{\mathbb{Z}} G:=\{p \in \operatorname{Spec} \mathbb{Z} \mid p$ appears in a minimal injective resolution of $G\}$.

Lemma 4.6. Let $A \in \mathcal{B}$. Then $A \otimes \kappa(p) \varsubsetneqq 0$ if and only if $p \in \operatorname{supp}_{\mathbb{Z}} \mathrm{K}_{*}(A)$.

Proof. First, assume $p \in \operatorname{supp}_{\mathbb{Z}} \mathrm{K}_{*}(A)$ and $p \neq 0$; that is, $\mathbb{Z}\left[\frac{1}{p}\right] / \mathbb{Z}$ appears in some degree as a direct summand of $M_{0}$ or $M_{1}$, where $\mathrm{K}_{*}(A) \hookrightarrow M_{0} \rightarrow M_{1}$ is a minimal injective resolution of $\mathbb{Z} / 2$-graded abelian groups. If it appears in $M_{0}$ in degree $k$, then $\operatorname{im}\left(\mathrm{K}_{*}(A)\right) \cap \Sigma^{k}\left(\mathbb{Z}\left[\frac{1}{p}\right] / \mathbb{Z}\right) \nsucceq\{0\}$ (here we use $\Sigma$ to denote the shift functor, in order not to confuse it with adjoining an element) because $M_{0}$ is an essential extension of $\mathrm{K}_{*}(A)$. So $\mathrm{K}_{*}(A)$ contains an isomorphic copy of $\Sigma^{k}\left(\mathbb{Z}\left[\frac{1}{p}\right] / \mathbb{Z}\right)$ or $\Sigma^{k}\left(\mathbb{Z} / p^{n}\right)$ for some $n \in \mathbb{N}$ (arbitrary subgroup of $\Sigma^{k}\left(\mathbb{Z}\left[\frac{1}{p}\right] / \mathbb{Z}\right)$ ). Thus $\mathrm{K}_{*}(A) \stackrel{p}{\rightarrow} \mathrm{K}_{*}(A)$ is not an isomorphism. Now if $\Sigma^{k}\left(\mathbb{Z}\left[\frac{1}{p}\right] / \mathbb{Z}\right)$ appears as a direct summand in $M_{1}$, but not in $M_{0}$, then $M_{0} \stackrel{p}{\rightarrow} M_{0}$ is an isomorphism. If we assume that $\mathrm{K}_{*}(A) \stackrel{p}{\rightarrow} \mathrm{K}_{*}(A)$ is also an isomorphism, then, by the Five Lemma, so is $M_{1} \stackrel{p}{\rightarrow} M_{1}$, which is a contradiction. So, if $p \neq 0$ and $p \in \operatorname{supp}_{\mathbb{Z}} \mathrm{K}_{*}(A)$ then $\mathrm{K}_{*}(A) \stackrel{p}{\rightarrow} \mathrm{K}_{*}(A)$ is not an isomorphism. Therefore, the lift of this map $A \stackrel{\tilde{p}}{\rightarrow} A$ is also not an isomorphism. So, cone $(A \stackrel{\tilde{p}}{\rightarrow} A) \cong A \otimes \kappa(p) \nsucceq 0$.

Conversely, if $A \otimes \kappa(p) \cong \operatorname{cone}(A \stackrel{\tilde{p}}{\rightarrow} A) \supsetneqq 0$, then $\mathrm{K}_{*}(A) \stackrel{p}{\rightarrow} \mathrm{K}_{*}(A)$ is not an isomorphism. By the Five Lemma, one of $M_{0} \stackrel{p}{\rightarrow} M_{0}$ or $M_{1} \stackrel{p}{\rightarrow} M_{1}$ is not an isomorphism as well; so $\mathbb{Z}\left[\frac{1}{p}\right] / \mathbb{Z}$ has to appear in some degree in $M_{0}$ or $M_{1}$.

Now consider $p=0$. Then $\mathbb{Q}$ appears in some degree $k$ as a direct summand of $M_{0}$ or $M_{1}$. In the first case, $\operatorname{im}\left(\mathrm{K}_{*}(A)\right) \cap \Sigma^{k}(\mathbb{Q}) \nsucceq\{0\}$, meaning that $\mathrm{K}_{*}(A)$ contains a torsion-free subgroup, thus $\mathrm{K}_{*}(A) \otimes \mathbb{Q} \nsucceq 0$. However, we also know that $\mathrm{K}_{*}(A \otimes \kappa(0)) \cong \mathrm{K}_{*}(A) \otimes \mathbb{Q}$ by the Künneth formula. If now $\mathbb{Q}$ does not appear in $M_{0}$, then $M_{0} \otimes \mathbb{Q} \cong 0$ and tensoring the minimal injective resolution with $\mathbb{Q}$ and using flatness of $\mathbb{Q}$, we conclude that also $M_{1} \otimes \mathbb{Q} \cong 0$ and thus $\mathbb{Q}$ does not appear as a direct summand in $M_{1}$ either.

Conversely, if $A \otimes \kappa(0) \varsubsetneqq 0$, then $\mathrm{K}_{*}(A \otimes \kappa(0)) \cong \mathrm{K}_{*}(A) \otimes \mathbb{Q} ¥ 0$. As above, tensoring the minimal injective resolution of $\mathrm{K}_{*}(A)$ with $\mathbb{Q}$, gives $M_{0} \otimes \mathbb{Q} \nsucceq 0$, so $0 \in \operatorname{supp}_{\mathbb{Z}} \mathrm{K}_{*}(A)$. 


\section{Localizing subcategories in the totally ordered case}

In this section, we restrict our attention to finite spaces with totally ordered lattice of open subsets. As observed in the preliminaries, this amounts to considering $X=\{1, \ldots, n\}$ totally ordered by $\leq$, where a subset is open if and only if it is of the form $[a, n]:=\{x \in X \mid a \leq x \leq n\}, a \in X$. Then locally closed subsets are those of the form $[a, b]$ with $a \leq b$ and $a, b \in X$. The set of non-empty locally closed subsets is denoted by $\mathbb{L} \mathbb{C}(X)$.

Definition 5.1. Let $\mathcal{L} \subseteq \mathcal{B}(X)$ be a localizing subcategory and $Y \in \mathbb{L} \mathbb{C}(X)$. Define $\mathrm{U}_{Y}^{\mathcal{L}} \subseteq$ Spec $\mathbb{Z}$ by

$$
\mathrm{U}_{Y}^{\mathcal{L}}:=\left\{p \in \operatorname{Spec} \mathbb{Z} \mid p \in \operatorname{supp}_{\mathbb{Z}} \mathrm{K}_{*}(A(Y)) \text { for some } A \in \mathcal{L}\right\} .
$$

Remark 5.2. In the introduction, we defined the support of an object $A \in \mathcal{L}$ in a localizing subcategory $\mathcal{L} \subseteq \mathcal{B}(X)$ as

$$
\operatorname{supp} A=\left\{(p, Y) \mid \mathrm{K}_{*}\left(A(Y) ; \mathbb{F}_{p}\right) \neq 0\right\},
$$

and the support of $\mathcal{L}$ as $\operatorname{supp} \mathcal{L}=\bigcup_{A \in \mathcal{L}} \operatorname{supp} A$.

If $A \in \mathcal{B}$, we may set $\mathrm{K}_{*}\left(A ; \mathbb{F}_{p}\right):=\mathrm{K}_{*}(A \otimes \kappa(p))$. Since the classical Künneth sequence for K-theory splits, $\mathrm{K}_{*}(A \otimes \kappa(p))$ is an $\mathbb{F}_{p^{-}}$-vector space.

Thus, by Lemma 4.6, for a localizing subcategory $\mathcal{L} \subseteq \mathcal{B}(X)$,

$$
\mathrm{U}_{Y}^{\mathcal{L}}=\{p \in \operatorname{Spec} \mathbb{Z} \mid(p, Y) \in \operatorname{supp} \mathcal{L}\} .
$$

We will prove that these sets are not independent: for any $Y \in \mathbb{L} \mathbb{C}(X)$ and $\mathcal{L} \subseteq \mathcal{B}(X)$ a localizing subcategory, if $p \in \mathrm{U}_{Y}^{\mathcal{L}}$ then there exists a maximal box

$$
B_{Z}=\left\{W \in \mathbb{L} \mathbb{C}(X) \mid \mathrm{K}_{*}\left(\mathcal{R}_{Z}(W)\right) \varsubsetneqq 0\right\}=\left\{W \in \mathbb{L} \mathbb{C}(X) \mid \mathrm{K}_{*}\left(\mathcal{R}_{Z}^{p}(W)\right) \varsubsetneqq 0\right\},
$$

such that $Y \in B_{Z}$ and $p \in \mathrm{U}_{V}^{\mathcal{L}}$ for all $V \in B_{Z}$. In other words, we have

Lemma 5.3. For every localizing subcategory $\mathcal{L} \subseteq \mathcal{B}(X)$ and $Y \in \mathbb{L} \mathbb{C}(X)$,

$$
\mathrm{U}_{Y}^{\mathcal{L}}=\bigcup_{\substack{Z \in \mathbb{L} C(X): \\ Y \in B_{Z}}} \bigcap_{V \in B_{Z}} \mathrm{U}_{V}^{\mathcal{L}} .
$$

Proof. First assume $p \in \bigcup_{Z: Y \in B_{Z}} \bigcap_{V \in B_{Z}} \mathrm{U}_{V}^{\mathcal{L}}$. Then $p \in \bigcap_{V \in B_{Z}} \mathrm{U}_{V}^{\mathcal{L}}$ for some $Z$ with $Y \in B_{Z}$. But then $\mathrm{U}_{Y}^{\mathcal{L}}$ is itself in this intersection. Thus $p \in \mathrm{U}_{Y}^{\mathcal{L}}$.

Now take $p \in \mathrm{U}_{Y}^{\mathcal{L}}$. By definition, there is $A \in \mathcal{L}$ with $p \in \operatorname{supp}_{\mathbb{Z}} \mathrm{K}_{*}(A(Y))$. Lemma 4.6 implies that $\operatorname{cone}(A(Y) \stackrel{\tilde{p}}{\rightarrow} A(Y)) \cong A(Y) \otimes \kappa(p) \nsucceq 0$. This implies that $\operatorname{cone}(A \stackrel{\tilde{p}}{\rightarrow} A) \nsucceq 0$ because $\operatorname{cone}(A \stackrel{\tilde{p}}{\rightarrow} A)(Y) \cong \operatorname{cone}(A(Y) \stackrel{\tilde{p}}{\rightarrow} A(Y))$. However, $\mathrm{FK}(\operatorname{cone}(A \stackrel{\tilde{p}}{\rightarrow} A))(Z) \cong \mathrm{K}_{*}(\operatorname{cone}(A \stackrel{\tilde{p}}{\rightarrow} A)(Z)) \cong \mathrm{K}_{*}(A(Z) \otimes \kappa(p))$ is an $\mathbb{F}_{p}$-vector space for any $Z \in \mathbb{L} \mathbb{C}(X)$ and $p \in \operatorname{Spec} \mathbb{Z}$ because the classical Künneth sequence for K-theory splits. Thus, by Corollary 3.44, there exists 
a multiset $I \subseteq \mathbb{L} \mathbb{C}(X)$ such that $\operatorname{FK}(\operatorname{cone}(A \stackrel{\tilde{p}}{\rightarrow} A)) \cong \bigoplus_{Z \in I} \operatorname{FK}\left(\mathcal{R}_{Z}^{p}\right) \cong$ $\operatorname{FK}\left(\bigoplus_{Z \in I} \mathcal{R}_{Z}^{p}\right)$.

Now we can use Corollary 3.39 and Theorem 3.37 to lift the isomorphism of filtrated K-theories to an isomorphism in $\mathcal{B}(X)$. In other words, $\operatorname{cone}(A \stackrel{\tilde{p}}{\rightarrow}$ $A) \cong \bigoplus_{Z \in I} \mathcal{R}_{Z}^{p}$. Since $\mathrm{K}_{*}(\operatorname{cone}(A \stackrel{\tilde{p}}{\rightarrow} A)(Y)) \not 0$, there is $Z \in I$ such that $\mathcal{R}_{Z}^{p}(Y) \not 0$. Since $\mathcal{L}$ is localizing, it contains all the direct summands of its objects. Thus $\mathcal{R}_{Z}^{p} \in \mathcal{L}$.

Since $\mathcal{R}_{Z}^{p}(V)=\left(\mathcal{R}_{Z} \otimes \kappa(p)\right)(V) \cong \mathcal{R}_{Z}(V) \otimes \kappa(p)$, the following implications hold for any $V \in \mathbb{L} \mathbb{C}(X)$ :

$$
\begin{aligned}
V \in B_{Z} & \Longleftrightarrow \mathrm{K}_{*}\left(\mathcal{R}_{Z}^{p}(V)\right) \nsucceq 0 \\
& \Longleftrightarrow \mathrm{K}_{*}\left(\mathcal{R}_{Z}^{p}(V)\right) \text { is isomorphic to } \mathbb{F}_{p}[i], i \in \mathbb{Z} / 2 \\
& \Longrightarrow p \in \operatorname{supp}_{\mathbb{Z}}\left(\mathrm{K}_{*}\left(\mathcal{R}_{Z}^{p}(V)\right)\right. \\
& \Longrightarrow p \in \mathrm{U}_{V}^{\mathcal{L}},
\end{aligned}
$$

where the first two equivalences hold because $\mathrm{K}_{*}\left(\mathcal{R}_{Z}(V) \otimes \kappa(p)\right) \cong \mathrm{K}_{*}\left(\mathcal{R}_{Z}(V)\right) \otimes$ $\mathrm{K}_{*}(\kappa(p))$ by to the Künneth formula and because $\mathrm{K}_{*}\left(\mathcal{R}_{Z}(V)\right) \cong \mathbb{Z}[i]$ for some $i \in \mathbb{Z} / 2$. In particular, these implications mean that $p \in \bigcap_{V \in B_{Z}} \mathrm{U}_{V}^{\mathcal{L}}$, and since $Y \in B_{Z}$, we get

$$
p \in \bigcup_{Z: Y \in B_{Z}} \bigcap_{V \in B_{Z}} \mathrm{U}_{V}^{\mathcal{L}} .
$$

Remark 5.4. Since $\mathrm{U}_{Y}^{\mathcal{L}}$ is itself in every intersection over which we are taking the unions in $\bigcup_{Z, Y \in B_{Z}} \bigcap_{V \in B_{Z}} \mathrm{U}_{V}^{\mathcal{L}}$, we can factor it out and get

$$
\mathrm{U}_{Y}^{\mathcal{L}}=\mathrm{U}_{Y}^{\mathcal{L}} \cap \bigcup_{\substack{Z \in \mathbb{L} \mathbb{C}(X): \\ Y \in B_{Z}}} \bigcap_{\substack{V \neq Y \\ V \in B_{Z}}} \mathrm{U}_{V}^{\mathcal{L}} .
$$

Therefore, Lemma 5.3 is equivalent to

$$
\mathrm{U}_{Y}^{\mathcal{L}} \subseteq \bigcup_{\substack{Z \in \mathbb{L} \mathbb{C}(X) \\ Y \in B_{Z}}} \bigcap_{\substack{V \neq Y \\ V \in B_{Z}}} \mathrm{U}_{V}^{\mathcal{L}}
$$

Definition 5.5. For a localizing subcategory $\mathcal{L} \subseteq \mathcal{B}(X)$ and $Y \in \mathbb{L} \mathbb{C}(X)$, define $\mathrm{V}_{Y}^{\mathcal{L}} \subseteq$ Spec $\mathbb{Z}$ by

$$
\mathrm{V}_{Y}^{\mathcal{L}}:=\left\{p \in \operatorname{Spec} \mathbb{Z} \mid \mathcal{R}_{Y}^{p} \in \mathcal{L}\right\} .
$$

Lemma 5.6. For any localizing subcategory $\mathcal{L} \subseteq \mathcal{B}(X)$,

$$
\mathcal{L} \cong\left\langle\mathcal{R}_{Y}^{p} \mid p \in \mathrm{V}_{Y}^{\mathcal{L}}, Y \in \mathbb{L} \mathbb{C}(X)\right\rangle .
$$

Proof. $\left\langle\mathcal{R}_{Y}^{p} \mid p \in \mathrm{V}_{Y}^{\mathcal{L}}, Y \in \mathbb{L} \mathbb{C}(X)\right\rangle \subseteq \mathcal{L}$ by definition.

Now if $A \in \mathcal{L}$, then $A \in\langle A \otimes \kappa(p) \mid p \in \operatorname{Spec} \mathbb{Z}\rangle$ by Lemma 4.5. But as was shown in the proof of Lemma $5.3, A \otimes \kappa(p) \cong \operatorname{cone}(A \stackrel{\tilde{p}}{\rightarrow} A) \cong \bigoplus_{Z \in I} \mathcal{R}_{Z}^{p}$ with $\mathcal{R}_{Z}^{p} \in \mathcal{L}$ for $Z \in I$. Thus also $A \in\left\langle\mathcal{R}_{Y}^{p} \mid p \in \mathrm{V}_{Y}^{\mathcal{L}}, Y \in \mathbb{L} \mathbb{C}(X)\right\rangle$. 
By Lemma 5.6, specifying the sets $\mathrm{V}_{Y}^{\mathcal{L}} \subseteq$ Spec $\mathbb{Z}$ for all $Y \in \mathbb{L} \mathbb{C}(X)$ completely determines the localizing subcategory $\mathcal{L}$. Our aim is to show that the sets $\mathrm{U}_{Y}^{\mathcal{L}}$ for all $Y \in \mathbb{L} \mathbb{C}(X)$ determine the sets $\mathrm{V}_{Y}^{\mathcal{L}}$, and thus $\mathcal{L}$ itself. However, in order to show this, we first need to prove some preliminary statements.

Lemma 5.7. Let $Y, V, W \in \mathbb{L} \mathbb{C}(X)$. If $Y$ equals $V \cap W$ or $V \cup W$ or $V \backslash W$, then $\mathcal{R}_{Y} \in\left\langle\mathcal{R}_{V}, \mathcal{R}_{W}\right\rangle$.

Proof. First, say $V \cup W \notin \mathbb{L} \mathbb{C}(X)$. This implies $V \backslash W=V$ and $V \cap W=\emptyset$, trivially giving the assertion. The same way, if $V \backslash W \notin \mathbb{L}(X)$, we must have $W \subset V$, thus $V \cup W=V$ and $V \cap W=W$, giving the result. Similarly, the assertion is trivial if $W \backslash V \notin \mathbb{L} \mathbb{C}(X)$. So we assume $V \cup W, V \backslash W, W \backslash V \in$ $\mathbb{L} \mathbb{C}(X)$. Write $V=\left[v_{1}, v_{2}\right]$ and $W=\left[w_{1}, w_{2}\right]$. Without loss of generality, we can also assume $v_{1} \leq w_{1}, v_{2} \leq w_{2}$ by exchanging $V$ and $W$ if necessary. However, since we sacrificed the symmetry, we have to prove the lemma for $Y=W \backslash V$ as well.

Let $Z \in \mathbb{L} \mathbb{C}(X)$ and $U \in \mathbb{O}(Z)$. By Lemma 3.29 this gives an exact triangle

$$
\Sigma \mathcal{R}_{U} \rightarrow \mathcal{R}_{Z \backslash U} \rightarrow \mathcal{R}_{Z} \rightarrow \mathcal{R}_{U}
$$

in $\mathfrak{K} \mathfrak{K}(X)$.

Since $W \backslash V=\left[v_{2}+1, w_{2}\right]$ is open in $V \cup W=\left[v_{1}, w_{2}\right], V \cap W=\left[w_{1}, v_{2}\right]$ is open in $V=\left[v_{1}, v_{2}\right]$ and $W=\left[w_{1}, w_{2}\right]$ is open in $V \cup W=\left[v_{1}, w_{2}\right]$, we get the following exact triangle

$$
\Sigma \mathcal{R}_{W \backslash V} \rightarrow \mathcal{R}_{V} \rightarrow \mathcal{R}_{V \cup W} \rightarrow \mathcal{R}_{W \backslash V}
$$

along with two exact triangles fitting in a commutative square

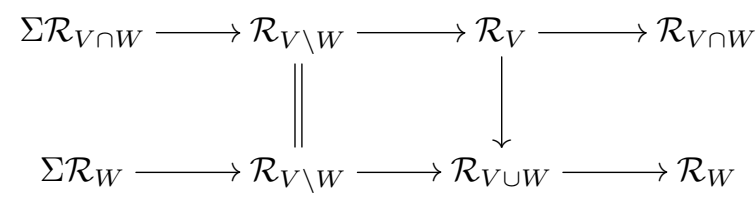

By the octahedral axiom, there exists a map $\mathcal{R}_{V \cap W} \rightarrow \mathcal{R}_{W}$ such that the third square in the above diagram will be homotopy cartesian; in other words, there is an exact triangle

$$
\Sigma \mathcal{R}_{W} \rightarrow \mathcal{R}_{V} \rightarrow \mathcal{R}_{V \cup W} \oplus \mathcal{R}_{V \cap W} \rightarrow \mathcal{R}_{W}
$$

These four triangles show that $\mathcal{R}_{W \backslash V}, \mathcal{R}_{V \cap W}, \mathcal{R}_{V \cup W}, \mathcal{R}_{V \backslash W} \in\left\langle\mathcal{R}_{V}, \mathcal{R}_{W}\right\rangle$.

Now we proceed to prove the key proposition.

Proposition 5.8. For a localizing subcategory $\mathcal{L} \subseteq \mathcal{B}(X)$, we have

$$
\mathrm{V}_{Y}^{\mathcal{L}}=\bigcap_{Z \in B_{Y}} \mathrm{U}_{Z}^{\mathcal{L}}
$$


Proof. If $p \in \mathrm{V}_{Y}^{\mathcal{L}}$, then $\mathcal{R}_{Y}^{p} \in \mathcal{L}$ by definition. Also, exactly as for Lemma 5.3,

$$
\begin{aligned}
Z \in B_{Y} & \Longleftrightarrow \mathrm{K}_{*}\left(\mathcal{R}_{Y}^{p}(Z)\right) \varsubsetneqq 0 \\
& \Longleftrightarrow \mathrm{K}_{*}\left(\mathcal{R}_{Y}^{p}(Z)\right) \text { is isomorphic to } \mathbb{F}_{p}[i] \text { for } i \in \mathbb{Z} / 2 \\
& \Longrightarrow p \in \operatorname{supp}_{\mathbb{Z}}\left(\mathrm{K}_{*}\left(\mathcal{R}_{Y}^{p}(Z)\right)\right. \\
& \Longrightarrow p \in \mathrm{U}_{Z}^{\mathcal{L}} .
\end{aligned}
$$

Thus $p \in \bigcap_{Z \in B_{Y}} \mathrm{U}_{Z}^{\mathcal{L}}$.

The opposite inclusion needs more work. Let $p \in \bigcap_{Z \in B_{Y}} \mathrm{U}_{Z}^{\mathcal{L}}$. As in the proof of Lemma 5.3, this means that for any $Z \in \mathbb{L} \mathbb{C}(X)$ with $Z \in B_{Y}$, there exists $W \in \mathbb{L} \mathbb{C}(X)$ with $Z \in B_{W}$ and $\mathcal{R}_{W}^{p} \in \mathcal{L}$. Let $J \subseteq \mathbb{L} \mathbb{C}(X)$ be the set of all such $W$ 's. Tensoring with $\kappa(p)$ is an exact functor and commutes with coproducts. So $\mathcal{R}_{Y} \in\left\langle\mathcal{R}_{W} \mid W \in J\right\rangle$ implies $\mathcal{R}_{Y}^{p} \in\left\langle\mathcal{R}_{W}^{p} \mid W \in J\right\rangle \subseteq \mathcal{L}$ and thus $p \in \mathrm{V}_{Y}^{\mathcal{L}}$. Therefore, it suffices to prove $\mathcal{R}_{Y} \in\left\langle\mathcal{R}_{W} \mid W \in J\right\rangle$.

First, we show that $Y$ is covered by intervals in $J$. Let $Y=[a, b]$. For any $i \in[a, b]$, by (3.4), we have $[1, i] \in B_{[a, b]}$ because $1 \leq a \leq i \leq b$. So we know that there exists $W \in J$ with $[1, i] \in B_{W}$. Let $W=\left[a_{1}, b_{1}\right]$. Since $[1, i] \in B_{\left[a_{1}, b_{1}\right]}$, again by (3.4), there is only one possibility, namely $1 \leq a_{1} \leq i \leq b_{1}$, which means $i \in W$.

Now, let $M^{i}$ be the interval of minimal length such that $i \in M^{i}$ and $\mathcal{R}_{M^{i}} \in\left\langle\mathcal{R}_{W} \mid W \in J\right\rangle$. Such an interval is unique; if $N^{i}$ is another interval with the same properties, then $i \in M^{i} \cap N^{i}, \mathcal{R}_{M^{i} \cap N^{i}} \in\left\langle\mathcal{R}_{W} \mid W \in J\right\rangle$ by Lemma 5.7 and $\left|M^{i} \cap N^{i}\right|<\left|M^{i}\right|$, contradicting minimality.

We want to demonstrate that $M^{i} \subseteq Y$; because then $Y=\bigcup_{j \in Y} M^{j}$, and by Lemma 5.7, $\mathcal{R}_{Y} \in\left\langle\mathcal{R}_{W} \mid W \in J\right\rangle$, concluding the proof of the proposition.

Let $M^{i}=[k, l]$. Assume $k<a$. Now, by (3.4), $[k+1, i] \in B_{[a, b]}$ because $k+1 \leq a \leq i \leq b$. Therefore, there exists $W \in J$ with $[k+1, i] \in B_{W}$. Let $W=[c, d]$. Again by (3.4), we have two possibilities:

Case $1 k+1 \leq c \leq i \leq d$. Then $[c, d] \cap[k, l]=[c, \min \{d, l\}]$, and thus $\mathcal{R}_{[c, \min \{d, l\}]} \in\left\langle\mathcal{R}_{W} \mid W \in J\right\rangle$ by Lemma 5.7. But $c \leq i \leq \min \{d, l\}$, thus $i \in[c, d] \cap[k, l]$. Moreover, $|[c, d] \cap[k, l]|<|[k, l]|$ because $k<c$ and $\min \{d, l\} \leq l$; this contradicts the minimality of $[k, l]$.

Case $2 c<k+1, d<i, k \leq d$. Then $[k, l] \backslash[c, d]=[d+1, l]$ because $c \leq k, d<i \leq l$. Thus $\mathcal{R}_{[d+1, l]} \in\left\langle\mathcal{R}_{W} \mid W \in J\right\rangle$ by Lemma 5.7. Since $d+1 \leq i \leq l, i \in[d+1, l]$. Moreover, $|[d+1, l]|<|[k, l]|$ because $k<d+1$; this contradicts the minimality of $[k, l]$.

We conclude that $a \leq k$. Assume $b<l$. Now, by $(3.4),[i+1, l] \in B_{[a, b]}$ because $a<i+1, b<l, i \leq b$. Therefore, there exists $W=[c, d] \in J$ with $[i+1, l] \in B_{[c, d]}$. Again, there are two cases to consider:

Case $1 i+1 \leq c \leq l \leq d$. Then $[k, l] \backslash[c, d]=[k, c-1]$ because $k<c, l \leq d$. Thus $\mathcal{R}_{[k, c-1]} \in\left\langle\mathcal{R}_{W} \mid W \in J\right\rangle$ by Lemma 5.7. Since $k \leq i \leq c-1$, $i \in[k, c-1]$. Moreover, $|[k, c-1]|<|[k, l]|$ because $c-1<l$; this contradicts the minimality of $[k, l]$. 
Case 2 $c<i+1, d<l, i \leq d$. Then $[c, d] \cap[k, l]=[\max \{k, c\}, d]$, and thus $\mathcal{R}_{[\max \{k, c\}, d]} \in\left\langle\mathcal{R}_{W} \mid W \in J\right\rangle$ by Lemma 5.7. But $\max \{k, c\} \leq i \leq d$, thus $i \in[c, d] \cap[k, l]$. moreover, $|[c, d] \cap[k, l]|<|[k, l]|$ because $d<l$; this contradicts the minimality of $[k, l]$.

Finally, we have $a \leq k \leq l \leq b$; that is, $M^{i} \subseteq Y$. This finishes the proof of the proposition.

Now we are ready to prove the main theorem of this section. We will restate it by concretely constructing the isomorphism. Let $m=|\mathbb{L} \mathbb{C}(X)|$ be the number of non-empty intervals in $X$; that is, if $X$ has $n$ points, $m=\frac{n(n+1)}{2}$.

Theorem 5.9. There is an inclusion-preserving isomorphism between localizing subcategories of $\mathcal{B}(X)$ and those elements $\left(\mathrm{U}_{Y_{1}}, \ldots, \mathrm{U}_{Y_{m}}\right) \in \mathcal{P}(\operatorname{Spec} \mathbb{Z})^{m}$ of the $m$-fold Cartesian product of subsets of the Zariski spectrum of the ring of integers, labeled by intervals $Y_{i} \subseteq X$, which satisfy $\mathrm{U}_{Y_{i}}=\bigcup_{j, Y_{i} \in B_{Y_{j}}} \bigcap_{Y_{k} \in B_{Y_{j}}} \mathrm{U}_{Y_{k}}$ for all $i=1, \ldots, m$. The isomorphism and its inverse map are given by

$$
\begin{aligned}
& \mathcal{L} \longmapsto\left\{\mathrm{U}_{Y_{i}}^{\mathcal{L}}\right\}_{i=1}^{m} \\
&\left\langle\mathcal{R}_{Y_{i}}^{p} \mid p \in \bigcap_{Y_{j} \in B_{Y_{i}}} \mathrm{U}_{Y_{j}}, i=1, \ldots, m\right\rangle \longleftrightarrow\left\{\mathrm{U}_{Y_{i}}\right\}_{i=1}^{m} .
\end{aligned}
$$

Proof. By Proposition 5.8, the sets $\mathrm{U}_{Y_{i}}^{\mathcal{L}}$ determine the sets $\mathrm{V}_{Y_{i}}^{\mathcal{L}}$ and therefore, by Lemma 5.6, the localizing subcategory $\mathcal{L}$.

It remains to show that if $\mathcal{L}=\left\langle\mathcal{R}_{Y_{i}}^{p} \mid p \in \bigcap_{Y_{j} \in B_{Y_{i}}} \mathrm{U}_{Y_{j}}, i=1, \ldots, m\right\rangle$, then $\mathrm{U}_{\mathrm{Y}_{\mathrm{i}}}=\mathrm{U}_{\mathrm{Y}_{\mathrm{i}}}^{\mathcal{L}}$ for all $i$.

Let $p \in \mathrm{U}_{Y_{i}}$, then $p \in \bigcup_{j, Y_{i} \in B_{Y_{j}}} \bigcap_{Y_{k} \in B_{Y_{j}}} \mathrm{U}_{Y_{k}}$. Therefore, there exists $j$ such that $\mathcal{R}_{Y_{j}}^{p} \in \mathcal{L}$ and $\mathcal{R}_{Y_{j}}^{p}\left(Y_{i}\right) \nsucceq 0$, since $Y_{i} \in B_{Y_{j}}$. This, in turn, implies that $p \in \operatorname{supp}_{\mathbb{Z}} \mathrm{K}_{*}\left(\mathcal{R}_{Y_{j}}^{p}\left(Y_{i}\right)\right)$. Thus $p \in \mathrm{U}_{Y_{i}}^{\mathcal{L}}$.

Now let $p \in \mathrm{U}_{Y_{i}}^{\mathcal{L}}$. Then, as in the proof of Lemma 5.3, there exists $j$ such that $\mathcal{R}_{Y_{j}}^{p} \in \mathcal{L}$ and $\mathcal{R}_{Y_{j}}^{p}\left(Y_{i}\right) \nsucceq 0$. It follows that for any set of generators of $\mathcal{L}$, at least one generator has to not vanish at $Y_{i}$ because $\mathcal{L}$ contains an object not vanishing at $Y_{i}$ and exact triangles in $\mathcal{L}$ come from short semi-split exact sequences of $\mathrm{C}^{*}$-algebras over $X$. In particular, there must exist $k$ with $p \in \bigcap_{Y_{l} \in B_{Y_{k}}} \mathrm{U}_{Y_{l}}$ and $\mathcal{R}_{Y_{k}}^{p} \in \mathcal{L}$. Since $Y_{i} \in B_{Y_{k}}$, we get $p \in \mathrm{U}_{Y_{i}}$.

Remark 5.10. Remark 5.2 identifies the set $\left\{\mathrm{U}_{Y_{i}}^{\mathcal{L}}\right\}_{i=1}^{m}$ with $\operatorname{supp} \mathcal{L}$. So Theorem 5.9 shows that every localizing subcategory is uniquely determined by its support and describes which sets can appear as the support of a localizing subcategory.

\subsection{Case of extensions}

To illustrate Theorem 5.9, let $X=\{1,2\}$ be the Sierpiński space, a two point topological space whose open sets are

$$
\mathbb{O}(X)=\{\emptyset,\{2\},\{1,2\}\} .
$$


5. Localizing subcategories in the totally ordered case

The category of $\mathrm{C}^{*}$-algebras over $X$ is equivalent to the category of extensions of $\mathrm{C}^{*}$-algebras. We have three non-empty locally closed sets, $\mathbb{L} \mathbb{C}(X)=$ $\{\{1\},\{2\},\{1,2\}\}$. The conditions on the sets $\mathrm{U}_{Y}$ for $Y \in \mathbb{L} \mathbb{C}(X)$ translate to

$$
\mathrm{U}_{\{1\}} \subseteq \mathrm{U}_{\{2\}} \cup \mathrm{U}_{\{1,2\}}, \quad \mathrm{U}_{\{2\}} \subseteq \mathrm{U}_{\{1\}} \cup \mathrm{U}_{\{1,2\}}, \quad \mathrm{U}_{\{1,2\}} \subseteq \mathrm{U}_{\{1\}} \cup \mathrm{U}_{\{2\}} .
$$

Therefore, we get:

Corollary 5.11. There is a bijection between localizing subcategories of the Kasparov category of extensions of $\mathrm{C}^{*}$-algebras and those triples of subsets of Spec $\mathbb{Z}$ which have the property that each one is inside the union of the other two. The bijection and its inverse map are given by

$$
\begin{aligned}
& \mathcal{L} \longmapsto\left(\begin{array}{c}
\left.\operatorname{supp}_{\mathbb{Z}} \mathrm{K}_{*}(I) \mid I \triangleleft A \in \mathcal{L}\right\} \\
\left\{\operatorname{supp}_{\mathbb{Z}} \mathrm{K}_{*}(A) \mid I \triangleleft A \in \mathcal{L}\right\} \\
\left\{\operatorname{supp}_{\mathbb{Z}} \mathrm{K}_{*}(A / I) \mid I \triangleleft A \in \mathcal{L}\right\}
\end{array}\right) \\
& \left\langle\begin{array}{c|c}
\kappa(p) \triangleleft \kappa(p) & p \in \mathrm{U}_{\{2\}} \cap \mathrm{U}_{\{1,2\}} \\
0 \triangleleft \kappa(q) & q \in \mathrm{U}_{\{1,2\}} \cap \mathrm{U}_{\{1\}} \\
\kappa(s)[1] \triangleleft 0 & s \in \mathrm{U}_{\{1\}} \cap \mathrm{U}_{\{2\}}
\end{array}\right) \longleftrightarrow\left(\begin{array}{c}
\mathrm{U}_{\{2\}} \\
\mathrm{U}_{\{1,2\}} \\
\mathrm{U}_{\{1\}}
\end{array}\right) .
\end{aligned}
$$

This example already demonstrates a difference between the classification of Theorem 5.9 and other instances in the literature, where the triangulated category $\mathfrak{T}$ in question carries an action of a commutative ring. In the latter case, as explained in the introduction, the lattice of localizing subcategories $\operatorname{Loc}(\mathfrak{T})$ is isomorphic to the lattice of subsets of some topological space $Y$, where, in addition, the topology on $Y$ determines certain structure on $\operatorname{Loc}(\mathfrak{T})$. In this case, one can regard $Y$ as a good candidate for a topological space associated to $\mathfrak{T}$. However, this construction is not possible for $\mathcal{B}(X)$.

Theorem 5.12. The lattice of localizing subcategories $\operatorname{Loc}(\mathcal{B}(X))$ of the bootstrap category $\mathcal{B}(X)$ is not isomorphic to the sublattice of a subset lattice $\mathcal{P}(S)$ for any set $S$.

Proof. Assume such an isomorphism:

$$
\phi: \operatorname{Loc}(\mathcal{B}(X)) \stackrel{\cong}{\longrightarrow} L,
$$

where $L \subseteq \mathcal{P}(S)$ is a sublattice of a subset lattice of some set $S$.

First, we want to show that we can assume $\phi(\langle 0\rangle)=\emptyset$, where $\langle 0\rangle$ denotes the trivial localizing subcategory. If this is not the case, let

$$
\widetilde{L}:=\{A \in \mathcal{P}(S \backslash \phi(\langle 0\rangle)) \mid A \cup \phi(\langle 0\rangle) \in L\} .
$$

Define $L \stackrel{\alpha}{\rightarrow} \widetilde{L}$ by $\alpha(A)=A \backslash \phi(\langle 0\rangle)$. Since $\phi$ is an isomorphism, $\phi(\langle 0\rangle)$ is a least element in $L$. It directly follows that $\alpha$ is a lattice isomorphism. So we can replace $\phi$ with $\alpha \circ \phi$ and consider $\widetilde{L}$ instead of $L$. However, $\alpha \circ \phi(\langle 0\rangle)=\emptyset$. So we may assume $\phi(\langle 0\rangle)=\emptyset$. 

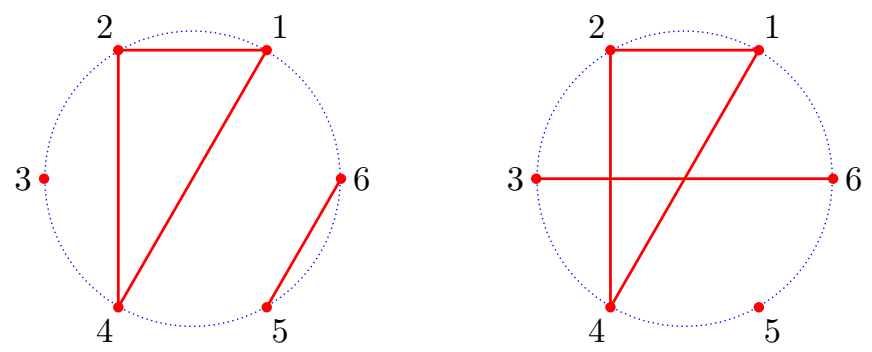

Figure 3.1: The first picture shows the noncrossing partition $\{\{1,2,4\},\{3\},\{5,6\}\}$ of the regular hexagon represented as vertices on a circle. The partition $\{\{1,2,4\},\{3,6\},\{5\}\}$ on the second picture is crossing.

By Corollary 5.11, elements of $\operatorname{Loc}(\mathcal{B}(X))$ are characterized by triples of subsets of Spec $\mathbb{Z}$, which have the property that each one is inside the union of the other two. In particular, for some prime $p \in \operatorname{Spec} \mathbb{Z}$, we have three localizing subcategories described by triples $(\{p\},\{p\}, \emptyset),(\emptyset,\{p\},\{p\})$ and $(\{p\}, \emptyset,\{p\})$. Let $\phi(\{p\},\{p\}, \emptyset)=A, \phi(\emptyset,\{p\},\{p\})=B$ and $\phi(\{p\}, \emptyset,\{p\})=C$ for some non-empty $A, B, C \in L$.

Since $\phi$ is order preserving and $(\{p\},\{p\}, \emptyset) \subset(\emptyset,\{p\},\{p\}) \vee(\{p\}, \emptyset,\{p\})$, we must have $A \subset B \cup C$. Hence $A \cap B \neq \emptyset$ or $A \cap C \neq \emptyset$. However, the only localizing subcategory that is contained in any two of the subcategories $(\{p\},\{p\}, \emptyset)$, $(\emptyset,\{p\},\{p\})$ and $(\{p\}, \emptyset,\{p\})$ is the trivial subcategory $\langle 0\rangle=(\emptyset, \emptyset, \emptyset)$; hence $(\{p\},\{p\}, \emptyset) \wedge(\emptyset,\{p\},\{p\})=(\emptyset, \emptyset, \emptyset)$ and $(\{p\},\{p\}, \emptyset) \wedge(\{p\}, \emptyset,\{p\})=(\emptyset, \emptyset, \emptyset)$. So $\phi((\{p\},\{p\}, \emptyset) \wedge(\emptyset,\{p\},\{p\})) \neq A \cap B$ and $\phi((\{p\},\{p\}, \emptyset) \wedge(\{p\}, \emptyset,\{p\})) \neq$ $A \cap C$. This contradicts the assumption that $\phi$ is a lattice isomorphism.

\section{Classification by noncrossing partitions}

In this section, describe the lattice of localizing subcategories of $\mathcal{B}(X)$ in another way, namely, by noncrossing partitions.

Definition 6.1. For $p \in \operatorname{Spec} \mathbb{Z}$, we say that the localizing subcategory $\mathcal{L}$ is $p$-local if, for all $Y \in \mathbb{L} \mathbb{C}(X)$, the set $\mathrm{U}_{Y}^{\mathcal{L}}$ is equal to $\{p\}$ or is empty.

Remark 6.2. The $p$-local localizing subcategories are exactly the ones generated by $\mathcal{R}_{Y}^{p}$ for $Y \in I \subseteq \mathbb{L} \mathbb{C}(X)$. Every localizing subcategory $\mathcal{L} \subseteq \mathcal{B}(X)$ can be uniquely represented by the $p$-local subcategories it contains, if we require that there is at most one (the largest) $p$-local subcategory for each $p \in \operatorname{Spec} \mathbb{Z}$ in this representation. This follows from Theorem 5.9 , since the corresponding property is trivial for the sets $\left(\mathrm{U}_{Y_{1}}^{\mathcal{L}}, \ldots, \mathrm{U}_{Y_{m}}^{\mathcal{L}}\right) \in \mathcal{P}(\operatorname{Spec} \mathbb{Z})^{m}$, where $m=|\mathbb{L} \mathbb{C}(X)|$ is the number of non-empty intervals. 


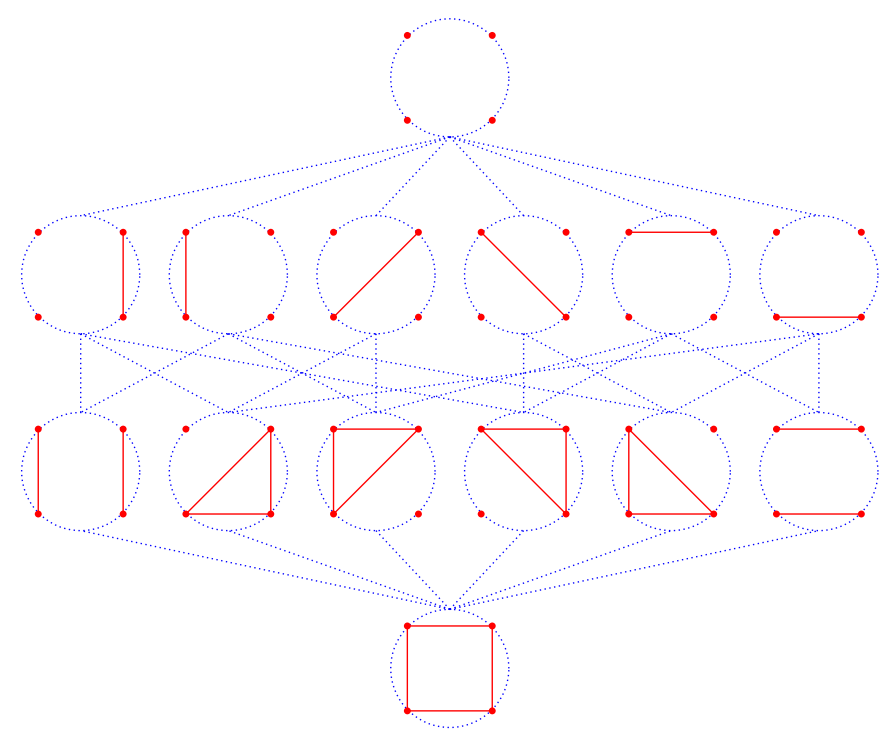

Figure 3.2: The lattice of noncrossing partitions of a square, that is, of the 4-element set. By Theorem 6.3, it corresponds to the lattice of all $p$-local localizing subcategories of $\mathcal{B}(\{1,2,3\})$.

\section{Classical noncrossing partitions}

A partition of a given set of $n$ elements is a collection of pairwise disjoint, nonempty subsets called blocks, whose union is the entire set. Since being in the same block is an equivalence relation, we denote it by $\sim$. A partition of $\{1, \ldots, n\}$ is noncrossing if, when four elements with $1 \leq a<b<c<d \leq n$ are such that $a \sim c$ and $b \sim d$, then the two blocks coincide, meaning $a \sim b \sim c \sim d$. The terminology comes from the fact that a noncrossing partition admits a planar representation as a partition of the vertices of a regular $n$-gon (labeled by $\{1, \ldots, n\})$ with the property that the convex hulls of its blocks are pairwise non-crossing (see Figure 3.1). The collection of noncrossing partitions of an $n$-element set is denoted by $\mathrm{NC}_{n}$.

$\mathrm{NC}_{n}$ becomes a partially ordered set when partitions are ordered by refinement: given partitions $\sigma, \tau \in \mathrm{NC}_{n}$, we say that $\tau \leq \sigma$ if each block of $\sigma$ is contained in a block of $\tau$. For each $n$, the partially ordered set $\mathrm{NC}_{n}$ is a self-dual, bounded lattice with $C_{n}$ elements, where $C_{n}=\frac{1}{n+1}\left(\begin{array}{c}2 n \\ n\end{array}\right)$ is the $n$th Catalan number. Figure 3.2 depicts this lattice for $n=4$. For the exposition of the classical theory of noncrossing partitions and the proof of these facts, we direct the interested reader to [1, Chapter 4].

\subsection{Classification}

Again let $X=\{1,2, \ldots, n\}$ with the Alexandrov topology. 
Theorem 6.3. There is a lattice isomorphism between p-local localizing subcategories of $\mathcal{B}(X)$ ordered by inclusion and $\mathrm{NC}_{n+1}$, the lattice of noncrossing partitions of a set with $n+1$ elements.

Proof. Denote the lattice of $p$-local localizing subcategories of $\mathcal{B}(X)$ by $L_{n}$. We are going to construct a lattice isomorphism

$$
\psi: L_{n} \stackrel{\sim}{\longrightarrow} \mathrm{NC}_{n+1} .
$$

By Theorem 5.9, a localizing subcategory $\mathcal{L} \in L_{n}$ is determined by the sets $\mathrm{U}_{[a, b]}^{\mathcal{L}}$ for $1 \leq a \leq b \leq n$. Given $\mathcal{L}$, we define a symmetric relation $\psi(\mathcal{L})$ on $\{1, \ldots, n+1\}$ by $a \sim b+1, b+1 \sim a \Longleftrightarrow \mathrm{U}_{[a, b]}^{\mathcal{L}}=\emptyset$ for $a \leq b$ and $a \sim a$ for all $a \in\{1, \ldots, n+1\}$.

We want to show that $\psi(\mathcal{L})$ is indeed a noncrossing partition. First, we prove transitivity. Let $a, b, c \in\{1, \ldots, n+1\}$ and $a \sim b, b \sim c$. If $a=b$ or $b=c$ or $a=c$ the assertion is trivial; so we assume they are all distinct. Define $x_{1}:=\min \{a, b, c\}, x_{3}:=\max \{a, b, c\}$ and let $x_{2}$ be the remaining third point. Thus $x_{1}<x_{2}<x_{3}$.

In the proof of Lemma 5.7, we showed that for $V \backslash W, W \backslash V, V \cup W \in \mathbb{L} \mathbb{C}(X)$ there is the following exact triangle in $\mathcal{B}(X)$ :

$$
\Sigma \mathcal{R}_{W \backslash V} \rightarrow \mathcal{R}_{V} \rightarrow \mathcal{R}_{V \cup W} \rightarrow \mathcal{R}_{W \backslash V} .
$$

Setting $V=\left[x_{1}, x_{2}-1\right]$ and $W=\left[x_{1}, x_{3}-1\right]$, and applying the functor $\mathrm{KK}_{*}(X ;-, A)$ for any $A \in \mathcal{B}(X)$ to this triangle, we get the six term exact sequence

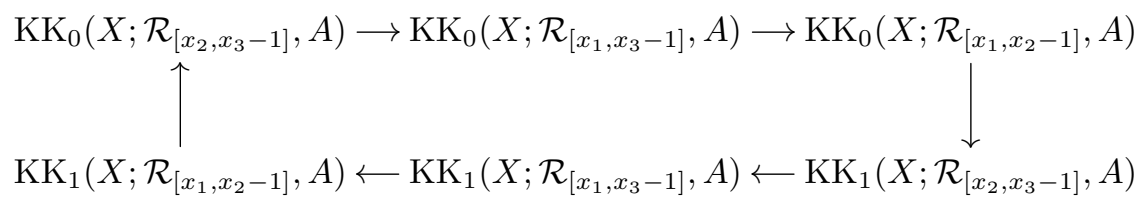

Theorem 3.27 gives $\mathrm{KK}_{*}\left(X ; \mathcal{R}_{Y}, A\right) \cong \mathrm{FK}_{Y}(A)=\mathrm{K}_{*}(A(Y))$. Hence

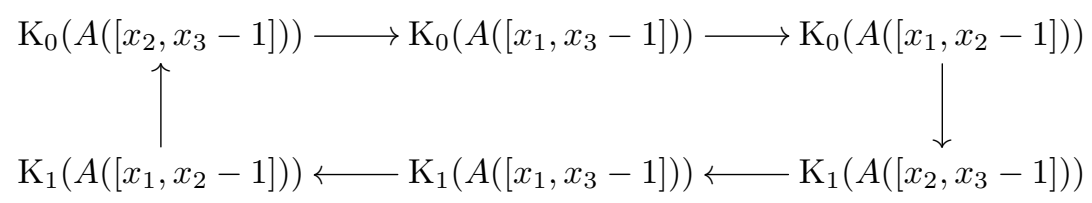

The exactness of the latter sequence implies

$\operatorname{supp}_{\mathbb{Z}} \mathrm{K}_{*}\left(A\left(\left[x_{2}, x_{3}-1\right]\right)\right) \subseteq \operatorname{supp}_{\mathbb{Z}} \mathrm{K}_{*}\left(A\left(\left[x_{1}, x_{2}-1\right]\right)\right) \cup \operatorname{supp}_{\mathbb{Z}} \mathrm{K}_{*}\left(A\left(\left[x_{1}, x_{3}-1\right]\right)\right)$, $\operatorname{supp}_{\mathbb{Z}} \mathrm{K}_{*}\left(A\left(\left[x_{1}, x_{3}-1\right]\right)\right) \subseteq \operatorname{supp}_{\mathbb{Z}} \mathrm{K}_{*}\left(A\left(\left[x_{2}, x_{3}-1\right]\right)\right) \cup \operatorname{supp}_{\mathbb{Z}} \mathrm{K}_{*}\left(A\left(\left[x_{1}, x_{2}-1\right]\right)\right)$, $\operatorname{supp}_{\mathbb{Z}} \mathrm{K}_{*}\left(A\left(\left[x_{1}, x_{2}-1\right]\right)\right) \subseteq \operatorname{supp}_{\mathbb{Z}} \mathrm{K}_{*}\left(A\left(\left[x_{1}, x_{3}-1\right]\right)\right) \cup \operatorname{supp}_{\mathbb{Z}} \mathrm{K}_{*}\left(A\left(\left[x_{2}, x_{3}-1\right]\right)\right)$. 

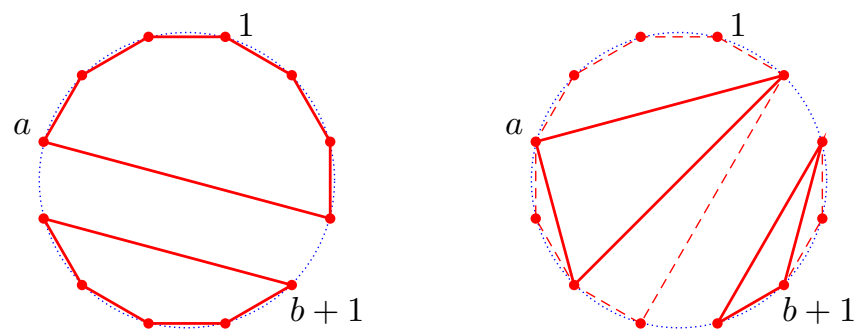

Figure 3.3: The first picture shows the decomposition into two connected blocks corresponding to the interval $[a, b]$. The second picture is an example of a "separating" decomposition (indicated by dashed lines) for a noncrossing partition drawn with bold lines.

Therefore, by definition $\mathrm{U}_{\left[x_{2}, x_{3}-1\right]}^{\mathcal{L}} \subseteq \mathrm{U}_{\left[x_{1}, x_{2}-1\right]}^{\mathcal{L}} \cup \mathrm{U}_{\left[x_{1}, x_{3}-1\right]}^{\mathcal{L}}, \mathrm{U}_{\left[x_{1}, x_{3}-1\right]}^{\mathcal{L}} \subseteq \mathrm{U}_{\left[x_{2}, x_{3}-1\right]}^{\mathcal{L}}$ $\cup \mathrm{U}_{\left[x_{1}, x_{2}-1\right]}^{\mathcal{L}}, \mathrm{U}_{\left[x_{1}, x_{2}-1\right]}^{\mathcal{L}} \subseteq \mathrm{U}_{\left[x_{1}, x_{3}-1\right]}^{\mathcal{L}} \cup \mathrm{U}_{\left[x_{2}, x_{3}-1\right]}^{\mathcal{L}}$. So for any distinct $i, j=$ $1, \ldots, 3$ such that $a=x_{i}$ and $c=x_{j}$, we get $\mathrm{U}_{[a, c-1]}^{\mathcal{L}}=\emptyset$; thus, $a \sim c$, proving that $\psi(\mathcal{L})$ is a partition.

Now let $1 \leq a<b<c<d \leq n+1$ and $a \sim c, b \sim d$; so $\mathrm{U}_{[a, c-1]}^{\mathcal{L}}=\emptyset$ and $\mathrm{U}_{[b, d-1]}^{\mathcal{L}}=\emptyset$. In the proof of Lemma 5.7, we also had a triangle

$$
\Sigma \mathcal{R}_{W} \rightarrow \mathcal{R}_{V} \rightarrow \mathcal{R}_{V \cup W} \oplus \mathcal{R}_{V \cap W} \rightarrow \mathcal{R}_{W} .
$$

After setting $V=[a, c-1]$ and $W=[b, d-1]$, the same argument as above gives that $\mathrm{U}_{[a, d-1]}^{\mathcal{L}} \cup \mathrm{U}_{[b, c-1]}^{\mathcal{L}} \subseteq \mathrm{U}_{[a, c-1]}^{\mathcal{L}} \cup \mathrm{U}_{[b, d-1]}^{\mathcal{L}}$. Thus $a \sim b \sim c \sim d$. So the partition $\psi(\mathcal{L})$ is a noncrossing.

If $\psi(\mathcal{L})=\psi\left(\mathcal{L}^{\prime}\right)$, then $\mathrm{U}_{Y}^{\mathcal{L}}=\mathrm{U}_{Y}^{\mathcal{L}^{\prime}}$ for all $Y \in \mathbb{L} \mathbb{C}(X)$ since $\mathcal{L}, \mathcal{L}^{\prime}$ are $p$-local. So $\mathcal{L}=\mathcal{L}^{\prime}$ by Theorem 5.9. So $\psi$ is injective.

Now we prove that $\psi$ is surjective. The subintervals of $[1, n]$ are in oneto-one correspondence with the decompositions of the $n+1$-gon into two (nonempty) connected subsets (see Figure 3.3). Here $[a, b] \in \mathbb{L} \mathbb{C}(X)$ corresponds to the decomposition into $[a+1, b+1]$ and its complement. Let $\mathcal{L}=\left\langle\mathcal{R}_{[a, b]}^{p}\right\rangle$. If $1 \leq x<y \leq n+1$, then (3.4) implies $x \nsim y$ in $\psi(\mathcal{L})$ if and only if $x \leq a<y \leq b+1$ or $a<x \leq b+1<y$. This exactly means that $\psi(\mathcal{L})$ is a decomposition of $n+1$-gon into two connected blocks (as in Figure 3.3). Since $\psi$ is injective, it gives a bijection between $p$-local localizing subcategories generated by a single interval and noncrossing partitions which are decompositions into two connected subsets.

Given a noncrossing partition $\sigma \in \mathrm{NC}_{n+1}, \psi^{-1}(\sigma)$ should be a $p$-local localizing subcategory $\mathcal{L}$ with $\mathrm{U}_{[a, b]}^{\mathcal{L}}=\emptyset$ if and only if $a \sim b+1$ in $\sigma$. For such an $\mathcal{L}$ to exist, we must show that this family of subsets $\mathrm{U}_{[a, b]}^{\mathcal{L}}$ satisfies the condition in Lemma 5.3. This is trivially satisfied if $\mathrm{U}_{[a, b]}=\emptyset$. If $\mathrm{U}_{[a, b]}=\{p\}$ then $a$ and $b+1$ are in different blocks of $\sigma$. By the noncrossing property, one finds another decomposition into two connected subsets (corresponding by $\psi$ 
to an interval $[c, d]$ ) such that it contains $\sigma$, and $a$ and $b+1$ are in different blocks of the decomposition. We call these decompositions "separating". To construct this, for example, one could move from vertex $a$ on the $n+1$-gon clockwise and counterclockwise connecting all vertices to $a$ along the way until the block of $b+1$ is reached, and connect all the remaining vertices to $b+1$ (see Figure 3.3). This implies that $[a, b] \in B_{[c, d]}$. Moreover, if $x \nsim y+1$ in the decomposition corresponding to $[c, d]$ then $x \nsim y+1$ also in $\sigma$. Hence if $[x, y] \in B_{[c, d]}$ then $\mathrm{U}_{[x, y]}=\{p\}$ for $\sigma$. In other words, the localization condition is satisfied. Therefore, $\psi$ is a bijection.

It is straightforward to see that $\psi$ and $\psi^{-1}$ are inclusion and refinement preserving, respectively. Therefore, $\psi$ is an isomorphism of lattices.

As a corollary, we get our main result

Theorem 6.4. The lattice of localizing subcategories of $\mathcal{B}(X)$ is isomorphic to $\prod_{p \in \operatorname{Spec} \mathbb{Z}} \mathrm{NC}_{n+1}^{p}$.

Proof. The statement directly follows from Theorem 6.3 and Remark 6.2.

\section{$7 \quad$ Algebraic analogue}

In this section, we use our techniques to classify localizing subcategories in a similar algebraic triangulated category. For the classification, we only used the structure of $\mathfrak{M o d}(\mathcal{N} \mathcal{T})_{c}$ and properties of the functor FK : $\mathfrak{K} \mathfrak{K}(X) \rightarrow \mathfrak{M o d}(\mathcal{N} \mathcal{T})_{c}$. Thus any triangulated category $\mathfrak{D}$ together with a functor $\mathrm{FH}: \mathfrak{D} \rightarrow \mathfrak{M o d}(\mathcal{N T})_{c}$ that satisfies analogous conditions to FK will have an isomorphic lattice of localizing subcategories. We will construct such a pair $(\mathfrak{D}, \mathrm{FH})$.

For $n \in \mathbb{N}$, let $\mathrm{A}_{n}$ denote the quiver

$$
n \rightarrow n-1 \rightarrow \cdots \rightarrow 2 \rightarrow 1 .
$$

Let $\mathbb{Z} \mathrm{A}_{n}$ denote the path ring of $\mathrm{A}_{n}$. This is the free Abelian group on the set of paths with multiplication defined by concatenation of paths when possible and zero otherwise (see Appendix A.2).

Remark 7.1. The ring $\mathbb{Z} \mathrm{A}_{n}$ is isomorphic to $T_{n}(\mathbb{Z})$, the ring of upper triangular $n \times n$-matrices with coefficients in $\mathbb{Z}$. An isomorphism $\phi: \mathbb{Z} \mathrm{A}_{n} \rightarrow T_{n}(\mathbb{Z})$ is defined as follows: for $1 \leq a \leq b \leq n$ let

$$
\phi(b, a)=E_{a, b},
$$

where $E_{a, b}$ is the $n \times n$-matrix with coefficient 1 in the intersection of the $a$-th row and the $b$-th column and all other coefficients zero.

A countable $\mathbb{Z}$-representation of a quiver is an assignment of a countable abelian group to every vertex and a map between the corresponding countable abelian groups to every edge. The category of countable $\mathbb{Z}$-representations of a quiver is equivalent to the category of countable right modules over the path ring. See Appendix A.2 for details. 
Now we consider the derived category $\mathfrak{D}:=\mathfrak{D e r}\left(\mathbb{Z} \mathrm{A}_{n} ; \mathbb{Z} / 2\right)_{c}$ of 2-periodic chain complexes over $\mathfrak{M o d}\left(\mathbb{Z} \mathrm{A}_{n}\right)_{c}$; in other words, the derived category with chain complexes $\left(G_{n}^{\bullet} \rightarrow \cdots \rightarrow G_{1}^{\bullet}, d^{\bullet}\right)$ of countable $\mathbb{Z}$-representations of $\mathbb{Z} \mathrm{A}_{n}$ as objects, which in addition satisfy $G_{a}^{i}=G_{a}^{i+2}$ and $d_{a}^{i}=d_{a}^{i+2}$ for all $i \in \mathbb{Z}, 1 \leq$ $a \leq n$, and where all maps between complexes are 2-periodic. So every $G \in \mathfrak{D}$ is of the form

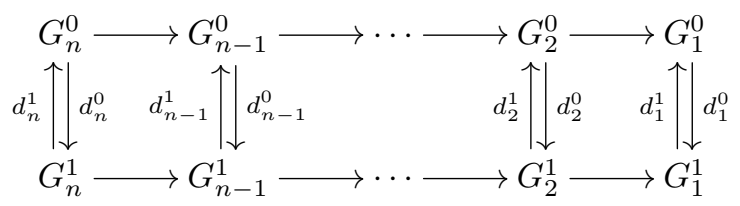

Definition 7.2. For $1 \leq a \leq n$, let $\mathcal{S}_{[a, n]} \in \mathfrak{D}$ be the object with $\mathcal{S}_{[a, n], i}=\mathbb{Z}[0]$ with identity maps in between if $1 \leq i \leq a$ and $\mathcal{S}_{[a, n], i}=0$ for $a<i \leq n$.

So $\mathcal{S}_{[a, n]}$ is of the form

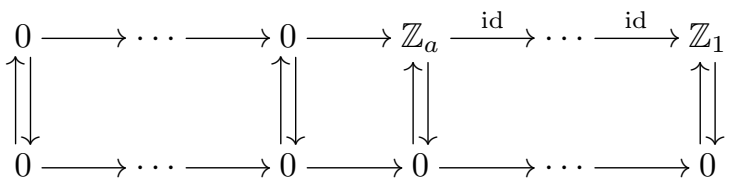

For $1 \leq a \leq b<n$, there is a natural map $\mathcal{S}_{[a, n]} \rightarrow \mathcal{S}_{[b+1, n]}$ given by identities and zeros appropriately. So we can define

Definition 7.3. For $1 \leq a \leq b<n$, let

$$
\mathcal{S}_{[a, b]}:=\operatorname{cone}\left(\mathcal{S}_{[a, n]} \rightarrow \mathcal{S}_{[b+1, n]}\right)[1] .
$$

So $\mathcal{S}_{[a, b]}$ is of the form

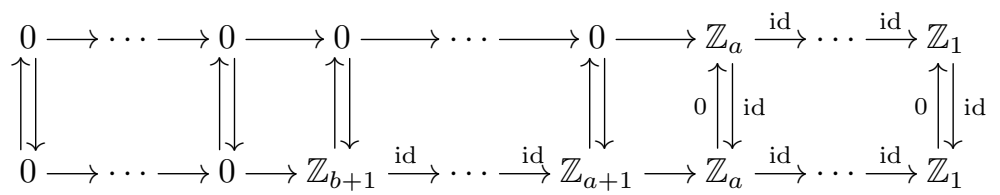

Lemma 7.4. If $1 \leq a \leq b \leq c \leq n$, then for any $G \in \mathfrak{D}$ there is a long exact sequence

$$
\cdots \rightarrow \mathfrak{D}\left(\mathcal{S}_{[b, c]}, G\right) \rightarrow \mathfrak{D}\left(\mathcal{S}_{[a, c]}, G\right) \rightarrow \mathfrak{D}\left(\mathcal{S}_{[a, b-1]}, G\right) \rightarrow \mathfrak{D}\left(\mathcal{S}_{[b, c]}, G\right)[1] \rightarrow \cdots,
$$

where $\mathfrak{D}(-, G)$ is the $\mathbb{Z} / 2$-graded Hom-functor.

Proof. If $c=n$, then there is an exact triangle

$$
\mathcal{S}_{[a, b-1]} \rightarrow \mathcal{S}_{[a, n]} \rightarrow \mathcal{S}_{[b, n]} \rightarrow \mathcal{S}_{[a, b-1]}[1]
$$


by definition of $\mathcal{S}_{[a, b]}$.

If $c<n$, we have a map of triangles

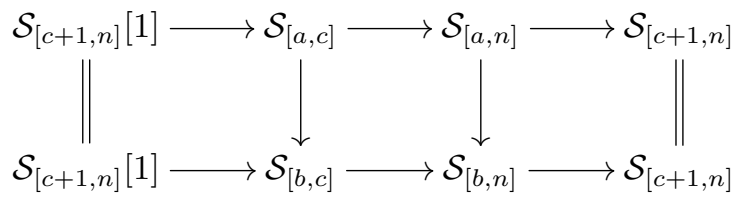

Therefore, the octahedral axiom gives $\operatorname{cone}\left(\mathcal{S}_{[a, c]} \rightarrow \mathcal{S}_{[b, c]}\right) \cong \operatorname{cone}\left(\mathcal{S}_{[a, n]} \rightarrow\right.$ $\left.\mathcal{S}_{[b, n]}\right)=\mathcal{S}_{[a, b-1]}[1]$, and we get an exact triangle in $\mathfrak{D}$

$$
\mathcal{S}_{[a, b-1]} \rightarrow \mathcal{S}_{[a, c]} \rightarrow \mathcal{S}_{[b, c]} \rightarrow \mathcal{S}_{[a, b-1]}[1] .
$$

In both cases, applying the functor $\mathfrak{D}(-, G)$ to the exact triangles (7.3) or (7.4) gives the desired long exact sequence.

Now we describe the homological functors represented by $\mathcal{S}_{[a, b]}$ for $1 \leq a \leq$ $b \leq n$. For this we define

Definition 7.5. Let $G \in \mathfrak{D}$. For $1 \leq a \leq n$ let

$$
\mathrm{FH}_{[a, n]}(G):=\mathrm{H}_{*}\left(G_{a}^{\bullet}\right),
$$

and for $1 \leq a \leq b<n$ let

$$
\mathrm{FH}_{[a, b]}(G):=\mathrm{H}_{*}\left(\operatorname{cone}\left(G_{b+1}^{\bullet} \rightarrow G_{a}^{\bullet}\right)\right) .
$$

Here the cone is taken in $\mathfrak{D e r}(\mathbb{Z})$, the derived category of abelian groups.

Lemma 7.6. There is a natural isomorphism

$$
\mathrm{FH}_{[a, b]}(G) \cong \mathfrak{D}\left(\mathcal{S}_{[a, b]}, G\right)
$$

for all $G \in \mathfrak{D}$ and $1 \leq a \leq b \leq n$.

Proof. For any ring $R$, the homology functor is representable on the derived category $\mathfrak{D e r}(R)$. In other words, for every $j \in \mathbb{Z}$ we have a natural Yoneda isomorphism

$$
\mathfrak{D e r}(R)(R[j],-) \cong \mathrm{H}_{j}(-), \quad f \mapsto \mathrm{H}_{j}(f)\left(1_{R}\right) .
$$

In our case, $\mathbb{Z} \mathrm{A}_{n}$ as a module over itself is represented by the diagram

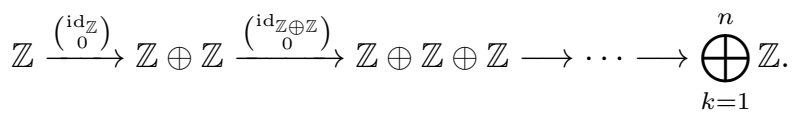

So $\mathbb{Z} \mathrm{A}_{n}[j] \cong \bigoplus_{k=1}^{n} \mathcal{S}_{[k, n]}[j]$ for $j \in \mathbb{Z} / 2$. By definition, $\mathrm{H}_{j}(G) \cong \bigoplus_{k=1}^{n} \mathrm{H}_{j}\left(G_{k}^{\bullet}\right)$ for any $G \in \mathfrak{D}$. Thus

$$
\mathrm{H}_{*}(G) \cong \bigoplus_{k=1}^{n} \mathrm{FH}_{[k, n]}(G) .
$$


Consider the composition

$$
\mathfrak{D}\left(\mathcal{S}_{[a, n]}[*], G\right) \hookrightarrow \mathfrak{D}\left(\mathbb{Z A}_{n}[*], G\right) \stackrel{\cong}{\longrightarrow} \mathrm{H}_{*}(G) \rightarrow \mathrm{FH}_{[a, n]}(G)
$$

given by

$$
f \mapsto f p_{[a, n]} \mapsto \mathrm{H}_{*}\left(f p_{[a, n]}\right)\left(1_{\mathbb{Z} \mathrm{A}_{n}}\right) \mapsto \pi_{[a, n]} \mathrm{H}_{*}\left(f p_{[a, n]}\right)\left(1_{\mathbb{Z A}_{n}}\right),
$$

where $p_{[a, n]}: \bigoplus_{k=1}^{n} \mathcal{S}_{[k, n]} \rightarrow \mathcal{S}_{[a, n]}$ and $\pi_{[a, n]}: \bigoplus_{k=1}^{n} \mathrm{FH}_{[k, n]}(G) \rightarrow \mathrm{FH}_{[a, n]}(G)$ are the projections onto direct summands. We claim that this is an isomorphism.

Since $f \in \mathfrak{D}\left(\mathcal{S}_{[a, n]}[*], G\right), \mathrm{H}_{*}\left(f p_{[a, n]}\right)$ is supported on those direct summands in (7.5) that correspond to paths in $\mathrm{A}_{n}$ starting at the vertex $a$. However, the element $1_{\mathbb{Z} A_{n}}$ corresponds to the sum of trivial paths for every vertex in $\mathrm{A}_{n}$. Thus $\mathrm{H}_{*}\left(f p_{[a, n]}\right)\left(1_{\mathbb{Z A}_{n}}\right)$ is exactly in the summand corresponding to the trivial path at $a$, which is the summand $\mathrm{FH}_{[a, n]}(G)$. Now, if we start with an element of $\mathrm{FH}_{[a, n]}(G)$, we get a map $f \in \mathfrak{D}\left(\mathbb{Z} \mathrm{A}_{n}[*], G\right)$ such that $\mathrm{H}_{*}(f)$ is supported on a summand corresponding to paths starting at $a$; this means that $f$ is in the summand $\mathfrak{D}\left(\mathcal{S}_{[a, n]}[*], G\right)$. So the composite is an isomorphism. Since all the maps are natural, so is their product.

Say now $1 \leq a \leq b<n$ and consider the exact triangle

$$
G_{b}^{\bullet} \longrightarrow G_{a}^{\bullet} \longrightarrow \operatorname{cone}\left(G_{b}^{\bullet} \rightarrow G_{a}^{\bullet}\right) \longrightarrow G_{b}^{\bullet}[1]
$$

in $\mathfrak{D e r}(\mathbb{Z})$. Applying the homology functor to this triangle gives the long exact sequence

$$
\cdots \rightarrow \mathrm{FH}_{[b, n]}(G) \rightarrow \mathrm{FH}_{[a, n]}(G) \rightarrow \mathrm{FH}_{[a, b-1]}(G) \rightarrow \mathrm{FH}_{[b, n]}(G)[1] \rightarrow \cdots
$$

Let $b<n$. By definition, $\mathrm{FH}_{[a, b]}\left(\mathcal{S}_{[a, b]}\right)=(\{(a,-a) \mid a \in \mathbb{Z}\}, 0)$. So there is the isomorphism $\mathrm{FH}_{[a, b]}\left(\mathcal{S}_{[a, b]}\right) \cong \mathbb{Z}[0]$ given by $((a,-a), 0) \mapsto(a, 0)$. Let $u=$ $((1,-1), 0)$ be the generator of $\mathrm{FH}_{[a, b]}\left(\mathcal{S}_{[a, b]}\right)$ corresponnding to $1 \in \mathbb{Z}[0]$ under this isomorphism. By Yoneda lemma, this gives a natural map $\mathfrak{D}\left(\mathcal{S}_{[a, b]}, G\right) \rightarrow$ $\mathrm{FH}_{[a, b]}(G)$ defined by

$$
f \mapsto \mathrm{FH}_{[a, b]}(f)(u) .
$$

So we have the commutative diagram

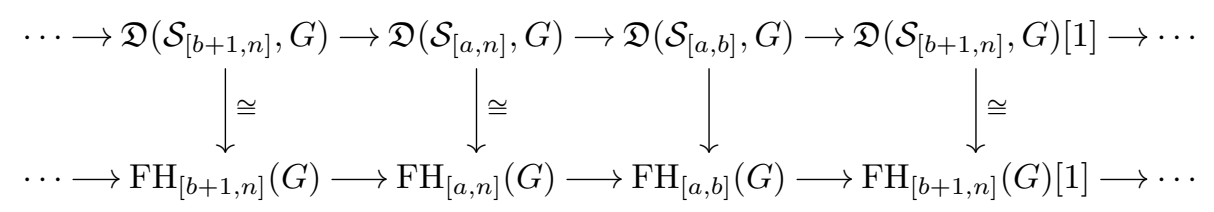

Its commutativity comes down to the commutativity of individual homomorphisms in chain maps; more precisely it follows from the definition of the objects $\mathcal{S}_{[a, b]}$ and the fact that $f \in \mathfrak{D}\left(\mathcal{S}_{[a, b]}, G\right)$ is represented by two chain maps, where one is a quasi-isomorphism. So we conclude the proof by the Five Lemma. 
Let $\mathbb{L} \mathbb{C}\left(\mathrm{A}_{n}\right)$ denote the set of all connected non-empty subquivers in $\mathrm{A}_{n}$ or, equivalently, non-empty intervals in $\{1, \ldots, n\}$.

Let $\mathrm{FH}_{-}(G)$ be an assignment that assigns a $\mathbb{Z} / 2$-graded abelian group $\mathrm{FH}_{[a, b]}(G)$ to every $[a, b] \in \mathbb{L} \mathbb{C}\left(\mathrm{A}_{n}\right)$. Now we prove that $\mathrm{FH}_{-}(G)$ can be enriched to a functor.

Lemma 7.7. $\mathrm{FH}_{-}(G)$ is an $\mathcal{N} \mathcal{T}$-module with projective resolution of length 1.

Proof. First, we prove that for any $G \in \mathfrak{D}$ there is an additive functor $\mathrm{FH}_{-}(G)$ : $\mathcal{N} \mathcal{T} \rightarrow \mathfrak{A b}^{\mathbb{Z} / 2}$. Take $[a, b],[c, d] \in \mathcal{N} \mathcal{T}$ with $\mathcal{N} \mathcal{T}_{*}([a, b],[c, d]) \cong \mathbb{Z}[0] ;$ then $c \leq a \leq d \leq b$ by conditions (3.4). If $d=b$, then the exact triangle (7.3) or (7.4) gives a map $\mathcal{S}_{[c, d]} \rightarrow \mathcal{S}_{[a, b]}$. If $d<b$, then $[a, d]=[a, b] \backslash[d+1, b]$ and again the exact triangle (7.3) or (7.4) gives canonical maps $\mathcal{S}_{[c, d]} \rightarrow \mathcal{S}_{[a, d]}$ and $\mathcal{S}_{[a, d]} \rightarrow \mathcal{S}_{[a, b]}$; after composing, we once again get the canonical map $\mathcal{S}_{[c, d]} \rightarrow \mathcal{S}_{[a, b]}$. So, in both cases, applying $\mathfrak{D}(-, G)$ gives the canonical map $\mathrm{FH}_{[a, b]}(G) \rightarrow \mathrm{FH}_{[c, d]}(G)$. We send the generator of $\mathcal{N} \mathcal{T}_{*}([a, b],[c, d])$ to this map.

If $\mathcal{N} \mathcal{T}_{*}([a, b],[c, d]) \cong \mathbb{Z}[1]$, then $c-1 \leq b$ and $a<c$ and $b<d$ by conditions (3.4). We already showed that this gives the maps $\mathrm{FH}_{[a, b]}(G) \rightarrow \mathrm{FH}_{[1, c-1]}(G)$ and $\mathrm{FH}_{[c, n]}(G) \rightarrow \mathrm{FH}_{[c, d]}(G)$. Since $[c, n]=[1, n] \backslash[1, c-1]$ the exact triangle (7.3) gives a canonical map $\mathrm{FH}_{[1, c-1]}(G) \rightarrow \mathrm{FH}_{[c, n]}(G)[1]$. After composing, we get the degree-one map

$$
\mathrm{FH}_{[a, b]}(G) \rightarrow \mathrm{FH}_{[1, c-1]}(G) \rightarrow \mathrm{FH}_{[c, n]}(G)[1] \rightarrow \mathrm{FH}_{[c, d]}(G)[1] .
$$

So indeed $\mathrm{FH}_{-}(G)$ is an $\mathcal{N} \mathcal{T}$-module.

The long exact sequence of Lemma 7.4 is the definition of exactness of an $\mathcal{N} \mathcal{T}$-module. So Lemma 7.6 shows that $\mathrm{FH}_{-}(G)$ is an exact module. By Theorem 3.37, this is equivalent to having a projective resolution of length 1.

Lemma 7.7 allows us to define

Definition 7.8. Filtrated homology is the functor

$$
\mathrm{FH}: \mathfrak{D} \rightarrow \mathfrak{M o d}(\mathcal{N} \mathcal{T})_{c}, \quad G \mapsto\left(\mathrm{FH}_{Y}(G)\right)_{Y \in \mathbb{L} \mathbb{C}\left(\mathrm{A}_{n}\right)} .
$$

The more precise reason why our classification result carries over from $\mathcal{B}(X)$ to $\mathfrak{D}$ is best explained in the context of relative homological algebra in a triangulated category recalled in Section 2. As was explained there, roughly speaking, having a homological ideal $\mathfrak{I}$ and a universal $\mathfrak{I}$-exact stable homological functor means that homological algebra in a target abelian category is the same as in a domain triangulated category with respect to $\mathfrak{I}$.

In our setup, we put

$$
\mathfrak{I}:=\bigcap_{Y \in \mathbb{L} \mathbb{C}\left(\mathrm{A}_{n}\right)} \operatorname{ker~FH}_{Y}=\operatorname{ker} \mathrm{FH} .
$$

In other words,

$$
\Im\left(G, G^{\prime}\right)=\left\{f \in \mathfrak{D}\left(G, G^{\prime}\right) \mid \mathrm{FH}_{Y}(f) \text { vanishes for all } Y \in \mathbb{L} \mathbb{C}\left(A_{n}\right)\right\} .
$$


Theorem 7.9. The functor $\mathrm{FH}: \mathfrak{D} \rightarrow \mathfrak{M o d}(\mathcal{N} \mathcal{T})_{c}$ is the universal $\mathfrak{I}$-exact stable homological functor.

Proof. We show that the theorem is a special case of a more general fact about relative homological algebra. Let $\mathfrak{C}$ be an at most countable set of objects in a triangulated category $\mathfrak{T}$ with countable coproducts. Let $\mathfrak{I}_{\mathfrak{C}}$ be the homological ideal defined as the kernel of the functor

$$
F_{\mathfrak{C}}: \mathfrak{T} \rightarrow \prod_{C \in \mathfrak{C}} \mathfrak{A b}^{\mathbb{Z}}, \quad A \mapsto(\mathfrak{T}(C, A))_{C \in \mathfrak{C}},
$$

where we assume that $F_{\mathfrak{C}}(A)$ is countable for all $A \in \mathfrak{T}$.

If we view $\mathfrak{C}$ as a $\mathbb{Z}$-graded pre-additive full subcategory of $\mathfrak{T}$, we get a corresponding category of countable graded right modules $\mathfrak{M o d}\left(\mathfrak{C}^{\mathrm{op}}\right)_{c}$. Then the enrichment of $F_{\mathfrak{C}}$ to the functor

$$
F_{\mathfrak{C}}^{\prime}: \mathfrak{T} \rightarrow \mathfrak{M o d}\left(\mathfrak{C}^{\mathrm{op}}\right)_{c},
$$

with the right $\mathfrak{C}$-module structure on $(\mathfrak{T}(C, A))_{C \in \mathfrak{C}}$ coming from composition of morphisms in $\mathfrak{T}$, is the universal $\mathfrak{I}_{\mathfrak{C}}$-exact stable homological functor $[32$, Theorem 4.4].

We apply this to our case, where $\mathfrak{T}=\mathfrak{D}, \mathfrak{C}=\left\{\mathcal{S}_{Y} \mid Y \in \mathbb{L} \mathbb{C}\left(\mathrm{A}_{n}\right)\right\}, F_{\mathfrak{C}}^{\prime}=\mathrm{FH}$ and $\mathfrak{I}_{\mathfrak{C}}=\mathfrak{I}$. Therefore, we only need to show that $\mathcal{N} \mathcal{T} \cong \mathfrak{C}^{\text {op }}$.

Let $[c, d] \in \mathbb{L} \mathbb{C}\left(\mathrm{A}_{n}\right)$. If $d=n$, the object $\mathcal{S}_{[c, d]} \in \mathfrak{D}$ is of the form (7.1). If $d<n$, by definition of the object $\mathcal{S}_{[c, d]}$, it is of the form (7.2). So for any $[a, b] \in \mathbb{L} \mathbb{C}\left(\mathrm{A}_{n}\right)$, computing appropriate homologies gives: for $d=n$, $\mathrm{FH}_{[a, b]}\left(\mathcal{S}_{[c, d]}\right)$ is isomorphic to $\mathbb{Z}[0]$ if $a \leq c \leq b$ and to zero otherwise; for $d<n$, $\mathrm{FH}_{[a, b]}\left(\mathcal{S}_{[c, d]}\right)$ is isomorphic to $\mathbb{Z}[0]$ if $a \leq c \leq b \leq d$, to $\mathbb{Z}[1]$ if $c+1 \leq a \leq d+1$, $d<b$ and to zero otherwise. Summing these up and using Theorem 7.6, we get

$$
\mathfrak{D}\left(\mathcal{S}_{[a, b]}, \mathcal{S}_{[c, d]}\right) \cong \mathrm{FH}_{[a, b]}\left(\mathcal{S}_{[c, d]}\right) \cong \begin{cases}\mathbb{Z}[0] & \text { if } a \leq c \leq b \leq d, \\ \mathbb{Z}[1] & \text { if } c<a \text { and } d<b \text { and } a-1 \leq d, \\ 0 & \text { otherwise. }\end{cases}
$$

which is exactly opposite to conditions (3.4). Thus we have an isomorphism of abelian groups $\mathcal{N} \mathcal{T}_{*}(Y, Z) \cong \mathfrak{D}\left(\mathcal{S}_{Z}, \mathcal{S}_{Y}\right)$ for all $Y, Z \in \mathbb{L} \mathbb{C}\left(\mathrm{A}_{n}\right)$.

Say $a \leq c \leq b \leq d$. There is a natural chain (diagram) map $\mathcal{S}_{[a, b]} \rightarrow \mathcal{S}_{[c, d]}$ given by identities and zeros appropriately. Denote this map by $\mu_{[a, b]}^{[c, d]}$. The map (7.6) or (7.7) maps $\mu_{[a, b]}^{[c, d]}$ to the generator of the group $\mathrm{FH}_{[a, b]}\left(\mathcal{S}_{[c, d]}\right) \cong \mathbb{Z}[0]$, and since $n \cdot \mu_{[a, b]}^{[c, d]} \neq 0$ for $n \in \mathbb{Z}, \mu_{[a, b]}^{[c, d]}$ generates the group $\mathfrak{D}\left(\mathcal{S}_{[a, b]}, \mathcal{S}_{[c, d]}\right)$. If $b<c$, then $\mathfrak{D}\left(\mathcal{S}_{[a, b]}, \mathcal{S}_{[c, d]}\right) \cong 0$ and we let $\mu_{[a, b]}^{[c, d]}$ be the zero map. With this convention, we get $\mu_{Z}^{W} \circ \mu_{Y}^{Z}=\mu_{Y}^{W}$ for all $Y, Z, W \in \mathbb{L} \mathbb{C}\left(\mathrm{A}_{n}\right)$, whenever all three maps are defined; this equality holds on the level of chain maps and thus also in the derived category. 
If $a-1 \leq d$, we get a degree-one chain map given by the composition

$$
\mathcal{S}_{[a, b]} \stackrel{\mu_{[a, b]}^{[a, n]}}{\longrightarrow} \mathcal{S}_{[a, n]} \stackrel{\delta}{\rightarrow} \mathcal{S}_{[1, a-1]}[1] \stackrel{\mu_{[1, a-1]}^{[a, n]}[1]}{\longrightarrow} \mathcal{S}_{[c, d]}[1],
$$

where $\delta$ is the boundary map coming from the triangle (7.3). Denote this chain map by $\delta_{[a, b]}^{[c, d]}$. If $c<a$ and $d<b$, the map (7.6) or (7.7) maps $\delta_{[a, b]}^{[c, d]}$ to the generator of the group $\mathrm{FH}_{[a, b]}\left(\mathcal{S}_{[c, d]}\right) \cong \mathbb{Z}[1]$. Since $\mathcal{S}_{[a, b], x}^{\bullet}$ is acyclic for $1 \leq x \leq a$, the map (7.6) or (7.7) maps $\delta_{[a, b]}^{[c, d]}$ to zero if $a \leq c$ or $b \leq d$. Thus $\delta_{[a, b]}^{[c, d]}$ vanishes in the derived category as predicted by the equation (7.8). Hence, if $a-1 \leq d$ and $c<a$ and $d<b, \delta_{[a, b]}^{[c, d]}$ generates $\mathfrak{D}\left(\mathcal{S}_{[a, b]}, \mathcal{S}_{[c, d]}\right)$.

By (7.8) any odd map from $\mathcal{S}_{[1, a-1]}$ vanishes. Since $\delta_{[a, b]}^{[c, d]}$ factors through $\mathcal{S}_{[1, a-1]}$, any product of degree-one morphisms is zero. In addition, for $Y, Z, W \in$ $\mathbb{L} \mathbb{C}\left(\mathrm{A}_{n}\right)$, a product $\mu_{Y}^{Z} \circ \delta_{W}^{Y}$ or $\delta_{Y}^{Z} \circ \mu_{W}^{Y}$ is equal to $\delta_{W}^{Z}$ whenever all three morphisms are defined, and zero otherwise; again, this equality holds on the level of chain maps and thus also in the derived category.

We conclude that the composition on $\mathfrak{C}^{\text {op }}$ coincides with the one on $\mathcal{N} \mathcal{T}$, proving that $\mathcal{N} \mathcal{T} \cong \mathfrak{C}^{\text {op }}$.

This allows us to state the Universal Coefficient Theorem:

Theorem 7.10. For any $G, K \in \mathfrak{D}$ and $j \in \mathbb{Z} / 2$, there are natural short exact sequences

$$
\operatorname{Ext}_{\mathcal{N} \mathcal{T}}^{1}(\mathrm{FH}(G)[j+1], \mathrm{FH}(K)) \hookrightarrow \mathfrak{D}(G, K)[j] \rightarrow \operatorname{Hom}_{\mathcal{N} \mathcal{T}}(\mathrm{FH}(G)[j], \mathrm{FH}(K)) .
$$

Proof. By Theorem 7.7, $\mathrm{FH}(G)$ has a projective resolution of length 1 for any $G \in \mathfrak{D}$.

Next, by definition of $\mathfrak{I}$ and Lemma 7.6

$$
\mathfrak{I}=\bigcap_{Y \in \mathbb{L} C\left(\mathrm{~A}_{n}\right)} \operatorname{ker} \mathfrak{D}\left(\mathcal{S}_{Y},-\right)=\operatorname{ker} \mathrm{FH} .
$$

Thus the objects $\left\{\mathcal{S}_{Y} \mid Y \in \mathbb{L} \mathbb{C}\left(\mathrm{A}_{n}\right)\right\}$ are $\mathfrak{I}$-projective. Now for any ring $R$, the derived category $\mathfrak{D e r}(R)$ is generated by the objects $\{R[i] \mid i \in \mathbb{Z}\}$. In our case, in the proof of Lemma 7.6, we showed that $\mathbb{Z} \mathrm{A}_{n}[j] \cong \bigoplus_{k=1}^{n} \mathcal{S}_{[k, n]}[j]$ for $j=0,1$. Therefore, the localizing subcategory generated by the $\mathfrak{I}$-projective objects is the whole $\mathfrak{D}$.

Since FH is the universal $\mathfrak{I}$-exact stable homological functor by Theorem 7.9, Theorem 2.12 now gives the desired short exact sequence.

Now we can also prove the analogue of Corollary 3.39.

Corollary 7.11. Let $M \in \mathfrak{M o d}(\mathcal{N} \mathcal{T})_{c}$ have a projective resolution of length 1 . Then there is $G \in \mathfrak{D}$ with $\mathrm{FH}(G) \cong M$, and this object is unique up to isomorphism in $\mathfrak{D}$. 
7. Algebraic analogue

Proof. Idempotent morphisms split in any triangulated category with countable coproducts (see [34]). Then Theorem 2.11 gives a length 1 projective resolution of $M$ of the form

$$
0 \rightarrow \mathrm{FH}\left(P_{1}\right) \stackrel{\mathrm{FH}(f)}{\longrightarrow} \mathrm{FH}\left(P_{0}\right) \rightarrow M \rightarrow 0,
$$

where $P_{1}, P_{0}$ are $\mathfrak{I}$-projective objects.

Embed the morphism $f$ in an exact triangle

$$
G[1] \stackrel{h}{\rightarrow} P_{1} \stackrel{f}{\rightarrow} P_{0} \stackrel{g}{\rightarrow} G .
$$

Since $\mathrm{FH}$ is homological and $\mathrm{FH}(f)$ is injective, we see that $\mathrm{FH}(h) \cong 0$ and $\mathrm{FH}(g)$ is surjective. Thus $\mathrm{FH}$ applied to the above triangle gives the short exact sequence

$$
0 \rightarrow \mathrm{FH}\left(P_{1}\right) \rightarrow \mathrm{FH}\left(P_{0}\right) \rightarrow \mathrm{FH}(G) \rightarrow 0 .
$$

Thus $\mathrm{FH}(G) \cong M$.

Now we prove the uniqueness. In the proof of Lemma 7.6 we showed that $\mathbb{Z A}_{n}[j] \cong \bigoplus_{k=1}^{n} \mathcal{S}_{[k, n]}[j]$ for $j \in \mathbb{Z} / 2$. However, $\mathrm{FH}(G) \cong \mathrm{FH}\left(G^{\prime}\right) \Longrightarrow$ $\mathrm{FH}_{[k, n]}(G) \cong \mathrm{FH}_{[k, n]}\left(G^{\prime}\right)$ for all $1 \leq k \leq n \Longrightarrow \mathfrak{D}\left(\mathcal{S}_{[k, n]}, G\right) \cong \mathfrak{D}\left(\mathcal{S}_{[k, n]}, G^{\prime}\right)$, for all $1 \leq k \leq n \Longrightarrow \mathfrak{D}\left(\bigoplus_{k=1}^{n} \mathcal{S}_{[k, n]}, G\right) \cong \mathfrak{D}\left(\bigoplus_{k=1}^{n} \mathcal{S}_{[k, n]}, G^{\prime}\right) \Longrightarrow$ $\mathfrak{D}\left(\mathbb{Z} \mathrm{A}_{n}[*], G\right) \cong \mathfrak{D}\left(\mathbb{Z A}_{n}[*], G^{\prime}\right) \Longrightarrow \mathrm{H}_{*}(G) \cong \mathrm{H}_{*}\left(G^{\prime}\right)$. But by Theorem 7.10 the isomorphism $\mathrm{FH}(G) \cong \mathrm{FH}\left(G^{\prime}\right)$ can be lifted to a morphism $f \in \mathfrak{D}\left(G, G^{\prime}\right)$; thus $f$ also induces the isomorphism $\mathrm{H}_{*}(G) \cong \mathrm{H}_{*}\left(G^{\prime}\right)$ and therefore is a quasiisomorphism. So $G \cong G^{\prime}$ in $\mathfrak{D}$.

To classify localizing subcategories in $\mathcal{B}(X)$, we used the existence of a tensor functor

$$
\otimes: \mathfrak{K} \mathfrak{K}(X) \times \mathfrak{K} \mathfrak{K} \rightarrow \mathfrak{K} \mathfrak{K}(X) .
$$

Similarly, in the case of $\mathfrak{D}$, we use the derived tensor product; in other words, we employ the biexact functor

$$
\otimes: \mathfrak{D} \times \mathfrak{D e r}(\mathbb{Z}) \rightarrow \mathfrak{D}
$$

which is associative and unital up to coherent isomorphism with respect to the derived tensor product in $\mathfrak{D e r}(\mathbb{Z})$.

In this setting, for $p \in \operatorname{Spec} \mathbb{Z}$, the objects $\mathbb{F}_{p}[0] \in \mathfrak{D e r}(\mathbb{Z})$ play the role of the objects $\kappa(p) \in \mathfrak{K} \mathfrak{K}$. For example, $\mathcal{S}_{Y}^{p}:=\mathcal{S}_{Y} \otimes \mathbb{F}_{p}[0]$ for $Y \in \mathbb{L} \mathbb{C}\left(\mathrm{A}_{n}\right)$.

Now we exhibit the analogues of the remaining the facts we used for our classification. Namely,

Corollary 7.12. Let $M \in \mathfrak{M o d}(\mathcal{N} \mathcal{T})_{\mathrm{c}}, p \in \operatorname{Spec} \mathbb{Z}$ and $j=0,1$. Then $M \cong \bigoplus_{Y \in I} \mathrm{FH}\left(\mathcal{S}_{Y}^{p}\right)\left[j_{Y}\right]$ for some (possibly countably infinite) multiset I with elements from $\mathbb{L} \mathbb{C}(X)$ if and only if $M(Y)$ is an $\mathbb{F}_{p}$-vector space for all $Y \in$ $\mathbb{L} \mathbb{C}\left(\mathrm{A}_{n}\right)$ and $M \cong \mathrm{FH}(G)$ for some $G \in \mathfrak{D}$. 
Proof. The result follows from Corollary 3.44, Theorem 3.37, Theorem 7.11 because

$$
\mathrm{FK}\left(\mathcal{R}_{Y}\right) \cong \mathrm{FH}\left(\mathcal{S}_{Y}\right)
$$

for all $p \in \operatorname{Spec} \mathbb{Z}$ and $Y \in \mathbb{L} \mathbb{C}\left(\mathrm{A}_{n}\right)$; this is true because $\mathrm{FK}_{Y}\left(\mathcal{R}_{Z}\right) \cong$ $\mathcal{N} \mathcal{T}_{*}(Z, Y) \cong \mathrm{FH}_{Y}\left(\mathcal{S}_{Z}\right)$ for all $Y, Z \in \mathbb{L} \mathbb{C}\left(\mathrm{A}_{n}\right)$ by $(7.8)$.

The analogues of Lemmas 4.5 and 4.6 are proven entirely similarly to their counterparts. We just replace $\kappa(-)$ by $h(-)$, where $h(G)=G[0]$ for $G \in \mathfrak{A b}_{c}^{Z / 2}$ is a unique object in $\mathfrak{D e r}(\mathbb{Z})$ with $\mathrm{H}_{*}(h(G)) \cong G[0], \mathrm{FK}_{Y}(-)$ by $\mathrm{FH}_{Y}(-)$ for $Y \in \mathbb{L} \mathbb{C}\left(\mathrm{A}_{n}\right)$, and where we replace exact triangles in $\mathcal{B}$ by appropriate exact triangles in $\mathfrak{D e r}(\mathbb{Z})$. This way, we have all the results we used, the support theory is identical, and finally we get

Theorem 7.13. The lattice of localizing subcategories of $\mathfrak{D e r}\left(\mathbb{Z} \mathrm{A}_{n} ; \mathbb{Z} / 2\right)_{c}$ is isomorphic to $\prod_{p \in \operatorname{Spec} \mathbb{Z}} \mathrm{NC}_{n+1}^{p}$.

Of course, Theorem 5.12 also carries over, and we find that the lattice of localizing subcategories does not recover any space from $\mathfrak{D e r}\left(\mathbb{Z} \mathrm{A}_{n} ; \mathbb{Z} / 2\right)_{c}$. 


\section{A. Appendices}

\section{A.1 Localization of a category}

In this section, we recall some aspects of localization theory for categories. All definitions and proofs can be found in [17].

\section{A.1.1 General localization}

For the theorem that follows, we use a more relaxed notion of a category where we allow morphisms not to be sets. In practice, however, one does not usually encounter any set theoretic issues.

Theorem A.1.1. Let $\mathfrak{C}$ be a category and let $\mathcal{S}$ be an arbitrary class of morphisms in $\mathfrak{C}$. Then there is a category $\mathfrak{C}\left[\mathcal{S}^{-1}\right]$ and a functor $Q: \mathfrak{C} \longrightarrow \mathfrak{C}\left[\mathcal{S}^{-1}\right]$ such that:

- $Q(s)$ is an isomorphism for each $s \in \mathcal{S}$;

- For any category $\mathfrak{E}$ and any functor $F: \mathfrak{C} \longrightarrow \mathfrak{E}$ such that $F(s)$ is an isomorphism for all $s \in \mathcal{S}$, there exists a functor $F_{\mathcal{S}}: \mathfrak{C}\left[\mathcal{S}^{-1}\right] \longrightarrow \mathfrak{E}$, and a natural isomorphism $F \cong F_{\mathcal{S}} \circ Q$. The functor $F_{\mathcal{S}}$ is unique up to natural isomorphism.

The category $\mathfrak{C}\left[\mathcal{S}^{-1}\right]$ is unique up to equivalence of categories.

We would like to sketch the construction of the category $\mathfrak{C}\left[\mathcal{S}^{-1}\right]$. Uniqueness is clear from the universal property.

Define objects of $\mathfrak{C}\left[\mathcal{S}^{-1}\right]$ as being the same as objects of $\mathfrak{C}$.

It remains to define morphism in $\mathfrak{C}\left[\mathcal{S}^{-1}\right]$. For any objects $A, B \in \mathfrak{C}$ a path from $A$ to $B$ of the length $n$ consists of:

(i) $n+1$ objects of $\mathfrak{C}$, such that $P_{0}=A, P_{1}, \ldots, P_{n-1}, P_{n}=B$

(ii) $n$ morphisms, one for each pair $\left(P_{i}, P_{i+1}\right)$, where either $f_{i}: P_{i} \rightarrow P_{i+1}$ can be any morphism in $\mathfrak{C}$, or $s_{i}: P_{i+1} \rightarrow P_{i}$ can only be a morphism from the class $\mathcal{S}$.

Now we define an equivalence relation $\sim$ on the set of all paths between $A$ and $B$. It is generated by

- for all $X, Y \in \mathfrak{C}$ and $f: X \rightarrow Y$ and $g: Y \rightarrow Z$,

$$
(X \stackrel{f}{\longrightarrow} Y \stackrel{g}{\longrightarrow} Z) \sim(X \stackrel{g \circ f}{\longrightarrow} Z)
$$


- for all $X, Y \in \mathfrak{C}$ and $s: X \rightarrow Y$ with $s \in \mathcal{S}$,

$$
(Y \stackrel{s}{\longleftarrow} X \stackrel{s}{\longrightarrow} Y) \sim\left(Y \stackrel{\operatorname{id}_{Y}}{\longrightarrow} Y\right)
$$

and

$$
(X \stackrel{s}{\longrightarrow} Y \stackrel{s}{\longleftarrow} X) \sim(X \stackrel{\operatorname{id} X}{\longrightarrow} X)
$$

- for all $X, Y \in \mathfrak{C}$ and $s: X \rightarrow Y$ with $s \in \mathcal{S}$,

$$
\left(Y \stackrel{\operatorname{id}_{Y}}{\longrightarrow} Y \stackrel{s}{\longleftarrow} X\right) \sim(Y \stackrel{s}{\longleftarrow} X)
$$

Morphisms between $A$ and $B$ in $\mathfrak{C}\left[\mathcal{S}^{-1}\right]$ are defined to be the equivalence classes of paths between $A$ and $B$. The composition is defined as concatenation, which clearly induces a composition on equivalence classes of paths.

The functor $Q$, called the localization functor, is defined to be the identity on objects, and to map the morphism $f: A \rightarrow B$ to the path $A \stackrel{f}{\longrightarrow} B$. Clearly, $Q(s)$ is invertible if $s \in \mathcal{S}$.

Let $\mathfrak{E}$ be as in the statement of the theorem. We define $F_{\mathcal{S}}(A):=F(A)$ for every object $A \in \mathfrak{C}\left[\mathcal{S}^{-1}\right]$. For a path $P$, consisting of $n$ morphisms $h_{1}, h_{2}, \ldots, h_{n}$, let

$$
F_{\mathcal{S}}(P):=F_{\mathcal{S}}\left(h_{n}\right) \circ \cdots \circ F_{\mathcal{S}}\left(h_{2}\right) \circ F_{\mathcal{S}}\left(h_{1}\right),
$$

where

$$
F_{\mathcal{S}}\left(h_{i}\right)= \begin{cases}F\left(h_{i}\right) & \text { if } h_{i}: P_{i} \rightarrow P_{i+1}, \\ F\left(h_{i}\right)^{-1} & \text { if } h_{i}: P_{i+1} \rightarrow P_{i} .\end{cases}
$$

It is now easy to see that the conditions of the theorem are satisfied.

\section{A.1.2 Calculus of fractions}

Let $\mathfrak{C}$ be a category. We concentrate on a special class of morphisms for localization in order to get more manageable results.

Definition A.1.2. We say that the class of morphisms $\mathcal{S}$ in $\mathfrak{C}$ admits a calculus of left fractions if the following are satisfied:

(CF1) For any object $X$ in $\mathfrak{C}$, the identity morphism id $X$ is in $\mathcal{S}$.

(CF2) If $s: X \rightarrow Y$ and $t: Y \rightarrow Z$ are in $\mathcal{S}$, then $t \circ s$ is also in $\mathcal{S}$.

(CF3) For each diagram $X^{\prime} \stackrel{s}{\leftarrow} X \stackrel{f}{\rightarrow} Y$ with $s \in \mathcal{S}$, there exists a commutative square

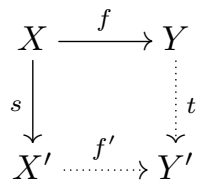

with $t \in \mathcal{S}$

(CF4) If $f, g: X \rightarrow Y$ are morphisms of $\mathfrak{C}$ and if $s: X^{\prime} \rightarrow X$ is a morphism of $\mathcal{S}$ with $f \circ s=g \circ s$, there exists a morphism $t: Y \rightarrow Y^{\prime}$ of $\mathcal{S}$ with $t \circ f=t \circ g$. 
The calculus of fractions gives special form to the morphisms in the localized category. Let $\mathfrak{C}\left[\mathcal{S}^{-1}\right]$ be the localization of $\mathfrak{C}$ with respect to $\mathcal{S}$, where $\mathcal{S}$ admits the calculus of left fractions. Let $Q: \mathfrak{C} \rightarrow \mathfrak{C}\left[\mathcal{S}^{-1}\right]$ be the localization functor constructed earlier.

By $(\mathrm{CF} 2), Q(t)^{-1} \circ Q(s)^{-1}=Q(s \circ t)^{-1}$ for $s, t \in \mathcal{S}$. Hence any morphism in $\left.\mathfrak{C}^{[} \mathcal{S}^{-1}\right]$ has the form $Q\left(f_{1}\right) \circ Q\left(s_{1}\right)^{-1} \circ \cdots \circ Q\left(f_{n}\right) \circ Q\left(s_{n}\right)^{-1}$, with $s_{i} \in \mathcal{S}$.

By (CF3), for any morphism $f$ and morphism $s \in \mathcal{S}$, there exist some morphism $g$ and a morphism $t \in \mathcal{S}$ with $f \circ t=s \circ g$, so that $Q(g) \circ Q(t)^{-1}=$ $Q(s)^{-1} \circ Q(f)$. Induction on $n$ gives that any morphism in $\mathfrak{C}\left[S^{-1}\right]$ is represented by a composition of two morphisms $Q(s)^{-1} \circ Q(f)$ with $s \in \mathcal{S}$.

The above observation leads us to consider the following: Let $\mathcal{S}$ admit a calculus of left fractions. Let us define the new category $\mathcal{S}^{-1} \mathfrak{C}$. Its objects are those of $\mathfrak{C}$. For any two objects $X$ and $Y$, consider the pairs of morphisms $(f, s)$ in $\mathfrak{C}$ of the form

$$
X \stackrel{f}{\longrightarrow} U \stackrel{s}{\longleftarrow} Y .
$$

If $s \in \mathcal{S}$, we call this pair a left fraction.

Now define the morphisms $X \rightarrow Y$ in $\mathcal{S}^{-1} \mathfrak{C}$ as equivalence classes $[f, s]$ of such left fractions, where two diagrams $\left(f_{1}, s_{1}\right)$ and $\left(f_{2}, s_{2}\right)$ are equivalent if the following commutative diagram exists with $s_{3} \in \mathcal{S}$

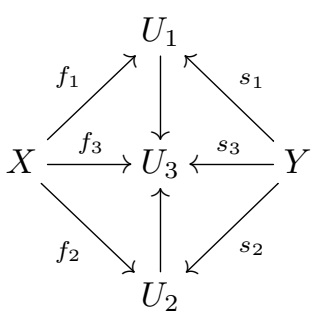

Define the composition of two equivalence classes $\left[f_{1}, s_{1}\right]$ and $\left[f_{2}, s_{2}\right]$ to be the equivalence class of $\left[f_{3} \circ f_{1}, s_{3} \circ s_{2}\right]$ where $f_{3}$ and $s_{3}$ are obtained from (CF3) as in the following commutative diagram:

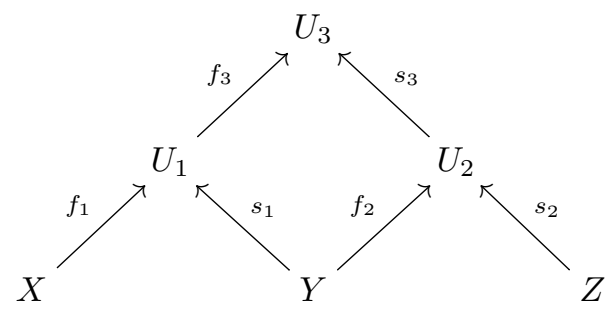

We have a canonical functor

$$
P: \mathfrak{C} \longrightarrow \mathcal{S}^{-1} \mathfrak{C}
$$

which is the identity on objects and takes the morphism $f: X \rightarrow Y$ to the equivalence class $\left[f, \operatorname{id}_{Y}\right]$. 
Let $\mathfrak{C}\left[\mathcal{S}^{-1}\right]$ be the localization of $\mathfrak{C}$ with respect to $\mathcal{S}$, and let $Q$ be the localization functor.

Proposition A.1.3. The functor $F: \mathcal{S}^{-1} \mathfrak{C} \longrightarrow \mathfrak{C}\left[\mathcal{S}^{-1}\right]$ which is the identity on objects and which takes a morphism $[f, s]$ to $Q(s)^{-1} \circ Q(f)$ is an isomorphism.

We say that a set of morphisms admits a calculus of right fractions if the dual conditions of (CF1)-(CF4) hold. All results about a set of morphisms admitting a calculus of left fractions have a dual version for a set of morphisms admitting a calculus of right fractions. If the class of morphisms satisfies both a calculus of left and a calculus of right fractions, we simply say that it satisfies a calculus of fractions.

\section{A.2 Quiver representations}

In this section, we very briefly recall basic definitions and constructions for quivers and their representations. The notions mentioned here can be found in $[2]$.

Definition A.2.1. A quiver $Q=\left(Q_{0}, Q_{1}, s, t\right)$ consists of a set of vertices $Q_{0}$, a set of edges (arrows) $Q_{1}$, and two maps

$$
s, t: Q_{1} \rightarrow Q_{0},
$$

which assign a source and target vertex to every edge, respectively.

Definition A.2.2. A $\mathbb{Z}$-representation of a quiver $Q$ is a collection $M=$ $\left\{M_{x}, M_{\alpha}\right\}_{x, \alpha}$, where $M_{x}$ is an abelian group for every vertex $x \in Q_{0}$ and $M_{\alpha}: M_{s(\alpha)} \rightarrow M_{t(\alpha)}$ is a homomorphism of abelian groups. A map between two representations $f: M \rightarrow M^{\prime}$ is a collection of homomorphisms $f_{x}: M_{x} \rightarrow M_{x}^{\prime}$ for every vertex $x \in Q_{0}$ such that the obvious diagrams commute.

This way, we get a category of quiver representations $\mathfrak{R e p}(Q, \mathbb{Z})$, which is nothing but a category of $Q$-shaped diagrams of abelian groups. In particular, considering $Q$ itself as a category, with vertices as objects and paths as morphisms, a $\mathbb{Z}$-representation of $Q$ is a covariant functor from $Q$ to the category of abelian groups. The maps of representations are precisely the natural transformations of the corresponding functors.

Definition A.2.3. Given a quiver $Q$, a path in $Q$ is a sequence of arrows $\left(\alpha_{1}, \ldots, \alpha_{n}\right)$ such that $s\left(\alpha_{i}\right)=t\left(\alpha_{i+1}\right)$ for $1 \leq i \leq n-1$. In addition, any vertex $x \in Q_{0}$ is considered as a path of length zero with source and target both $x$; it is denoted by $e_{x}$.

Definition A.2.4. The path ring $\mathbb{Z} Q$ of a quiver $Q$ is the ring (possibly non-unital) generated by all paths in $Q$, where multiplication is given by concatenation of paths when possible and by zero otherwise. 
In general, the path ring of a quiver only has an approximate identity. The path ring of a quiver $Q$ is unital if and only if $Q$ has finitely many vertices, that is, $Q_{0}$ is finite. In this case, the unit is given by the sum $\Sigma_{x \in Q_{0}} e_{x}$.

In certain cases, modules over a path ring can be characterized as representations of a quiver and vice versa. We recall this construction in the case which is relevant to us.

Theorem A.2.5. Let $Q$ be a quiver with finitely many vertices. The category of $\mathbb{Z}$-representations $\mathfrak{R e p}(Q, \mathbb{Z})$ of the quiver $Q$ is equivalent to the category $\mathfrak{M o d}(\mathbb{Z} Q)$ of (right) $\mathbb{Z} Q$-modules.

Given a $\mathbb{Z}$-representation $\left\{M_{x}, M_{\alpha}\right\}_{x, \alpha}$ of $Q$, let $M=\bigoplus_{x \in Q_{0}} M_{x}$. This way, $M$ becomes a $\mathbb{Z} Q$-module with obvious multiplication: given a path $\alpha$ with $s(\alpha)=x$ and $t(\alpha)=y$ define

$$
\alpha \cdot b= \begin{cases}\alpha b & \text { if } b \in M_{x}, \\ 0 & \text { otherwise }\end{cases}
$$

This also gives maps of modules from maps of $\mathbb{Z}$-representations. For a map $\left\{f_{x}\right\}_{x}:\left\{M_{x}, M_{\alpha}\right\}_{x, \alpha} \rightarrow\left\{M_{x}^{\prime}, M_{\alpha}^{\prime}\right\}_{x, \alpha}$, we just take a direct sum $f=\bigoplus_{x} f_{x}$ : $\bigoplus_{x} M_{x} \rightarrow \bigoplus_{x} M_{x}^{\prime}$.

Given a $\mathbb{Z} Q$-module $M \in \mathfrak{M}$ od $(\mathbb{Z} Q)$, put $M_{x}=e_{x} M$ for every $x \in Q_{0}$; for an arrow $\alpha \in Q_{1}$ with $s(\alpha)=x$ and $t(\alpha)=y$, let $M_{\alpha}: M_{x} \rightarrow M_{y}$ be the multiplication with $\alpha$.

For a $\mathbb{Z} Q$-module homomorphism $f: M \rightarrow M^{\prime}$, let $f_{x}=\left.f\right|_{M_{x}}$. Then, since $f$ is a module map, we get $f=\bigoplus_{x} f_{x}: \bigoplus_{x} M_{x} \rightarrow \bigoplus_{x} M_{x}^{\prime}$.

Straightforward computations show that these constructions are inverse to each other. 



\section{Bibliography}

[1] Drew Armstrong. Generalized Noncrossing Partitions and Combinatorics of Coxeter Groups. Mem. Amer. Math. Soc. American Mathematical Society, Providence, R.I., 2009.

[2] Ibrahim Assem, Andrzej Skowronski, and Daniel Simson. Elements of the Representation Theory of Associative Algebras, I. London Mathematical Society Texts, 2006.

[3] Paul Balmer. The spectrum of prime ideals in tensor triangulated categories. J. Reine Angew. Math, 588:149-168, 2005.

[4] Apostolos Beligiannis. Relative homological algebra and purity in triangulated categories. J. Algebra, 227(1):268-361, 2000.

[5] Dave Benson, Srikanth B. Iyengar, and Henning Krause. Local cohomology and support for triangulated categories. Ann. Sci. Éc. Norm. Supér., 41:573-619, 2008.

[6] Dave Benson, Srikanth B. Iyengar, and Henning Krause. Stratifying modular representations of finite groups. Ann. of Math., 174:1643-1684, 2011.

[7] Bruce Blackadar. K-Theory for Operator Algebras. Springer-Verlag, New York, first edition, 1986.

[8] Alexander Bonkat. Bivariante K-Theorie für Kategorien projektiver Systeme von $C^{*}$-Algebren. $\mathrm{PhD}$ thesis, Westf. Wilhelms-Universität Münster, 2002.

[9] J. Daniel Christensen. Ideals in triangulated categories: Phantoms, ghosts and skeleta. Adv. in Math., 136(2):284-339, 1998.

[10] John Dauns and Karl Heinrich Hofmann. Representation of rings by sections. Mem. Amer. Math. Soc., (83):12-180, 1968.

[11] Kenneth R. Davidson. $C^{*}$-Algebras by Example, volume 6. Fields Institute Monographs, 1996.

[12] Ivo Dell'Ambrogio. Localizing subcategories in the bootstrap category of separable C*-algebras. J. K-theory, 8:493-505, 2011.

[13] Ethan S. Devinatz, Michael J. Hopkins, and Jeffrey H. Smith. Nilpotence and stable homotopy theory I. Ann. of Math., 128(2):207-241, 1988. 
[14] Albrecht Dold and Dieter Puppe. Homologie nicht-additiver Funktoren. Anwendungen. Ann. Inst. Fourier, 11:201-312, 1961.

[15] J.M.G. Fell. The structure of algebras of operator fields. Acta Mathematica, 106(3-4):233-280, 1961.

[16] Peter Freyd. Representations in abelian categories. In Proceedings of the Conference on Categorical Algebra, pages 95-120. Springer Berlin Heidelberg, 1966.

[17] Peter Gabriel and Michel Zisman. Calculus of Fractions and Homotopy Theory. Springer-Verlag Berlin, New York, 1967.

[18] Sergei I. Gelfand and Yuri I. Manin. Methods of Homological Algebra. Springer-Verlag Berlin Heidelberg, second edition, 2003.

[19] Nigel Higson. A characterization of KK-theory. Pacific J. Math., 126(2):253276, 1987.

[20] Michael J. Hopkins. Global methods in homotopy theory. In Homotopy Theory: Proceedings of the Durham Symposium 1985, pages 73-96. Cambridge University Press, 1987.

[21] Michael J. Hopkins and Jeffrey H. Smith. Nilpotence and stable homotopy theory II. Ann. of Math., 148(1):1-49, 1998.

[22] Gennadi G. Kasparov. Equivariant KK-theory and the Novikov conjecture. Inventiones mathematicae, 91(1):147-201, 1988.

[23] Bernhard Keller. Derived categories and their uses. In Handbook of algebra, volume 1, pages 671-701. North-Holland, Amsterdam, 1996.

[24] Claudia Köhler. A combinatorial classification of thick subcategories of derived and stable categories. PhD thesis, Bielefeld University, 2011.

[25] Joseph Lipman. Notes on derived functors and Grothendieck duality, foundations of Grothendieck duality for diagrams of schemes. In Lecture Notes in Math. 1960, pages 1-259. Springer, 2009.

[26] Eben Matlis. Injective modules over Noetherian rings. Pacific J. Math., 8(3):511-528, 1958.

[27] Ralf Meyer. Categorical aspects of bivariant K-theory. In K-theory and Noncommutative Geometry, EMS Series of Congress Reports, pages 1-39. European Mathematical Society, Zürich, 2008.

[28] Ralf Meyer. Homological algebra in bivariant K-theory and other triangulated categories. II. Tbilisi Math. J., 1:165-210, 2008.

[29] Ralf Meyer and Ryszard Nest. The Baum-Connes conjecture via localisation of categories. Topology, 45(2):209-259, 2006. 
[30] Ralf Meyer and Ryszard Nest. $\mathrm{C}^{*}$-algebras over topological spaces: the bootstrap class. Münster J. Math., 2:215-252, 2009.

[31] Ralf Meyer and Ryszard Nest. Homological algebra in bivariant K-theory and other triangulated categories. I. In Triangulated Categories, pages 236-289. Cambridge University Press, 2010.

[32] Ralf Meyer and Ryszard Nest. C*-algebras over topological spaces: Filtrated K-theory. Canad. J. Math., 64:368-408, 2012.

[33] Amnon Neeman. The chromatic tower for D(R). Topology, 31(3):519-532, 1992.

[34] Amnon Neeman. Triangulated Categories, volume 148 of Ann. of Math. Studies. Princeton University Press, 2001.

[35] Manuel L. Reyes. Obstructing extensions of the functor spec to noncommutative rings. Israel J. of Math., 192(2):667-698, 2012.

[36] Jonathan Rosenberg and Claude Schochet. The Künneth theorem and the universal coefficient theorem for Kasparov's generalized K-functor. Duke Math. J., 55(2):431-474, 061987.

[37] Jun Tomiyama. Topological representation of $\mathrm{C}^{*}$-algebras. Tohoku Math. J. (2), 14(2):187-204, 1962.

[38] Jean-Louis Verdier. Categories dérivées. Quelques résultats (etat 0). In Cohomologie Etale, volume 569 of Lecture Notes in Mathematics, pages 262-311. Springer, Berlin Heidelberg, 1977.

[39] Charles A. Weibel. An Introduction to Homological Algebra. Cambridge Studies in Advanced Mathematics. Cambridge University Press, 1995. 